\title{
Determination of Heat Purgeable and Ambient Purgeable Volatile Organic Compounds in Water by Gas Chromatography/Mass Spectrometry
}

Chapter 12 of

Section B, Methods of the National Water Quality Laboratory, of Book 5, Laboratory Analysis

Techniques and Methods 5-B12 



\section{Determination of Heat Purgeable and Ambient Purgeable Volatile Organic Compounds in Water by Gas Chromatography/Mass Spectrometry}

By Donna L. Rose, Mark W. Sandstrom, and Lucinda K. Murtagh

Chapter 12 of

Section B, Methods of the National Water Quality Laboratory, of Book 5, Laboratory Analysis

Techniques and Methods 5-B12 


\section{U.S. Department of the Interior SALLY JEWELL, Secretary}

\section{U.S. Geological Survey Suzette M. Kimball, Director}

\section{U.S. Geological Survey, Reston, Virginia: 2016}

For more information on the USGS - the Federal source for science about the Earth, its natural and living resources, natural hazards, and the environment—visit http://www.usgs.gov or call 1-888-ASK-USGS.

For an overview of USGS information products, including maps, imagery, and publications, visit http://store.usgs.gov/.

Electronic version of the standard operating procedure for the methods is available upon request from LabHelp@usgs.gov.

Any use of trade, firm, or product names is for descriptive purposes only and does not imply endorsement by the U.S. Government.

Although this information product, for the most part, is in the public domain, it also may contain copyrighted materials as noted in the text. Permission to reproduce copyrighted items must be secured from the copyright owner.

Suggested citation:

Rose, D.L., Sandstrom, M.W., and Murtagh, L.K., 2016, Determination of heat purgeable and ambient purgeable volatile organic compounds in water by gas chromatography/mass spectrometry: U.S. Geological Survey Techniques and Methods, book 5, chap. B12, 61 p., http://dx.doi.org/10.3133/tm5B12.

ISSN 2328-7055 (online) 


\section{Contents}

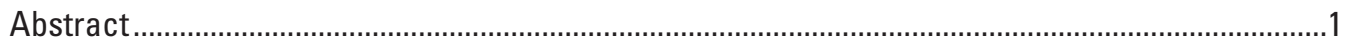

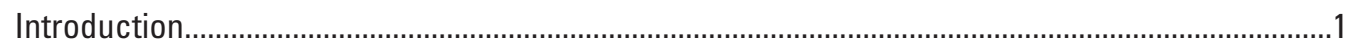

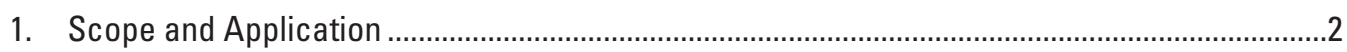

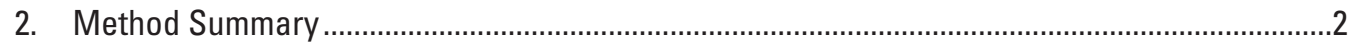

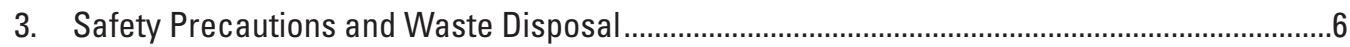

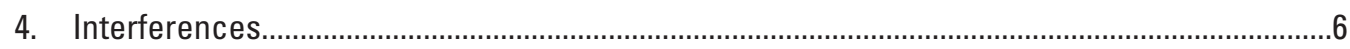

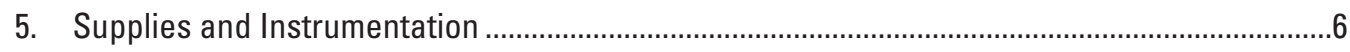

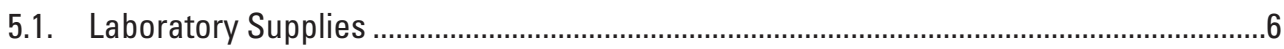

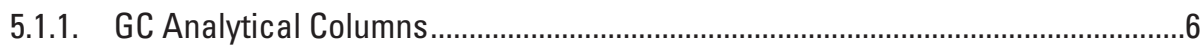

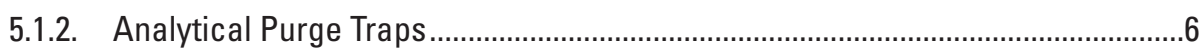

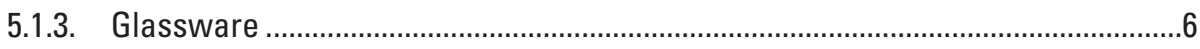

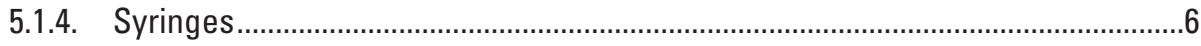

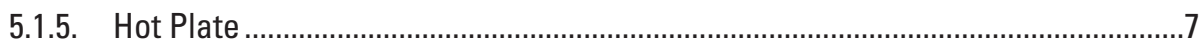

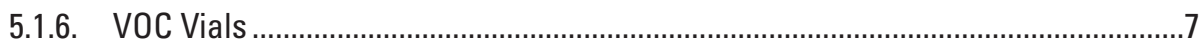

5.2. Sample Collection Supplies .......................................................................................

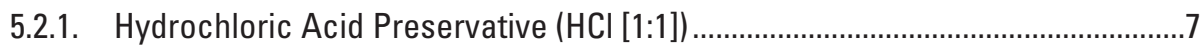

5.2.2. Trip Blanks.........................................................................................................

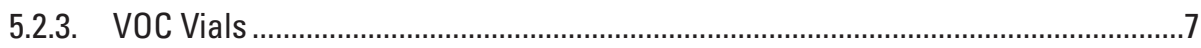

5.2.4. VOC Vials with Dechlorination Reagent...........................................................7

5.2.5. Water, Nitrogen Purged, Organic Blank …………...............................................

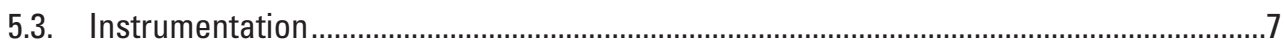

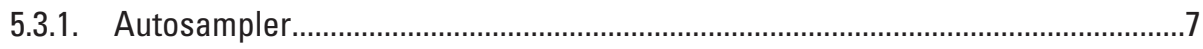

5.3.2. Purge-and-Trap Sample Concentrator ………...................................................

5.3.3. Gas Chromatograph............................................................................................

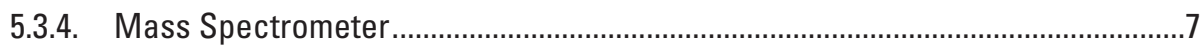

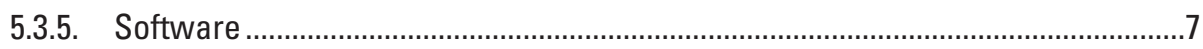

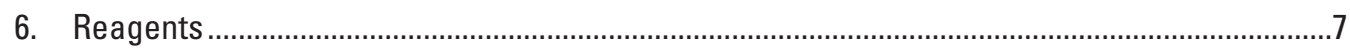

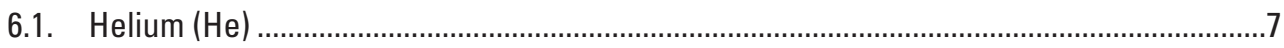

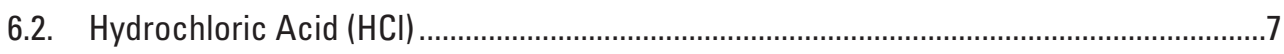

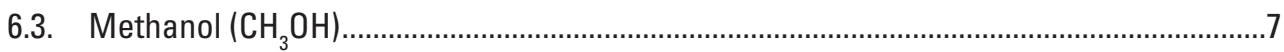

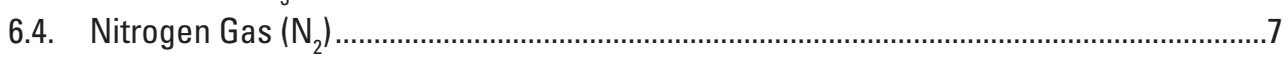

6.5. Water $\left(\mathrm{H}_{2} \mathrm{O}\right)$, Volatile Blank (VBW) .......................................................................

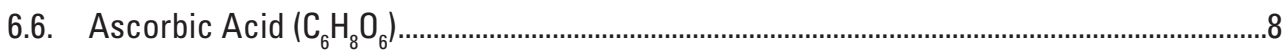

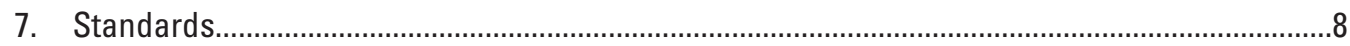

7.1. Mass Spectrometer Performance Evaluation Standard Solution .....................................8

7.2. Surrogate and Internal Standard Compound Solution, Method 0-4437...........................8

7.3. Surrogate and Internal Standard Compound Solution, Method 0-4436............................

7.4. Calibration Standard Solutions, Method 0-4437 …………….........................................8

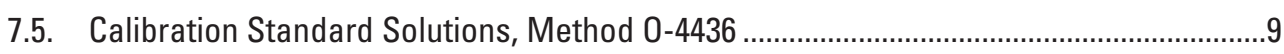

7.6. Third Party Check Standard Solution ..........................................................................

7.7. Laboratory Reagent-Water Spike Standard Solution .......................................................... 
8. Sample Collection, Preservation, Labeling, Shipment, and Holding Times ..............................

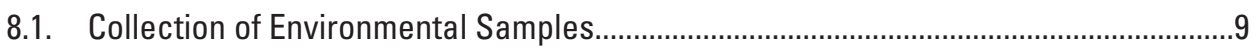

8.2. Collection of Field Quality Control Samples …….....................................................

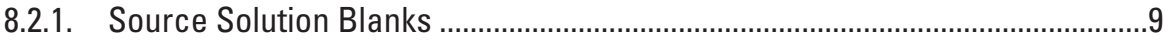

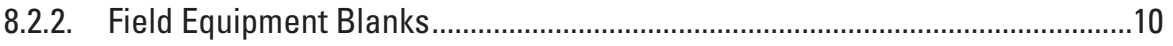

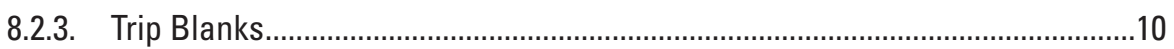

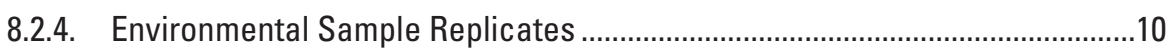

8.2.5. Environmental Samples for Laboratory Matrix Spikes .....................................10

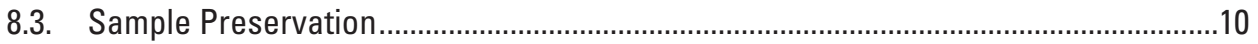

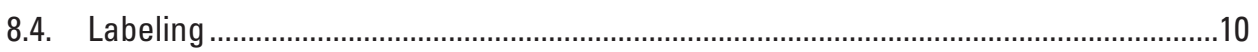

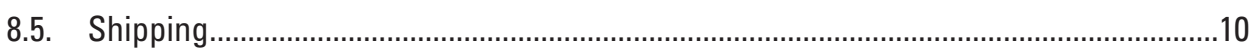

8.6. Holding Times ………..........................................................................................

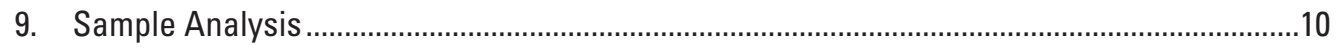

9.1. Method Configuration ................................................................................................11

9.1.1. Instrument Operating Conditions .....................................................................11

9.1.2. Internal Standard and Surrogate Standard Compound Assignments, Method 0-4437..........................................................................................11

9.1.3. Internal Standard and Surrogate Standard Compound Assignments, Method 0-4436..........................................................................................11

9.1.4. Compound Retention Times ........................................................................11

9.1.5. Mass Spectrometry Tune Check ....................................................................11

9.1.6. Initial Instrument Check ................................................................................11

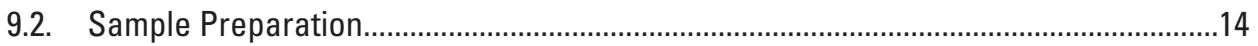

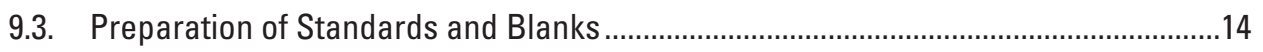

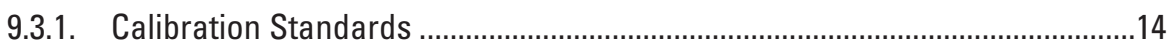

9.3.2. Continuing Calibration Verification Standard....................................................14

9.3.3. Third Party Check Standard ............................................................................14

9.3.4. Laboratory Reagent-Water Spike Standard .................................................14

9.3.5. Limit of Quantitation Standard ..........................................................................14

9.3.6. Laboratory Matrix Spike ....................................................................................

9.3.7. Laboratory Reagent-Water Blank ...................................................................14

9.3.8. Carryover Blank ............................................................................................14

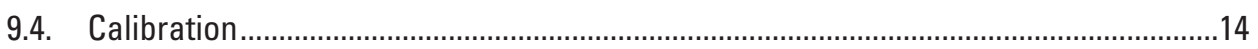

9.4.1. Prepare an Initial Calibration Curve ………….............................................14

9.4.2. Calculate the Response Factor ...................................................................15

9.4.3. Evalute the Calibration Curve for Each Compound ……….............................15

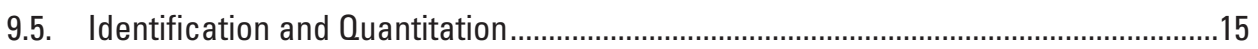

10. Quality Assurance and Quality Control Samples and Criteria ................................................16

10.1. Continuing Calibration Verification Standard ………...............................................

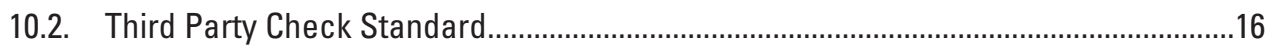

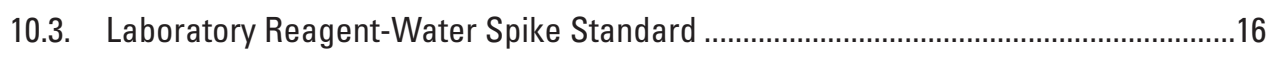

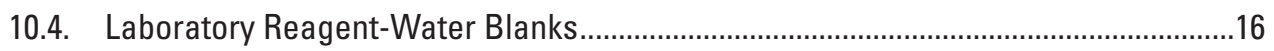

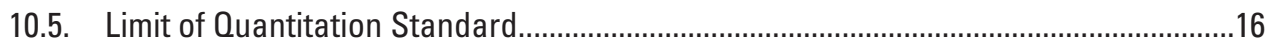

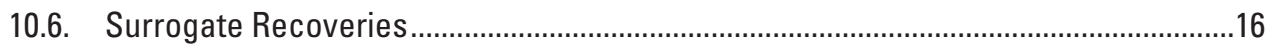

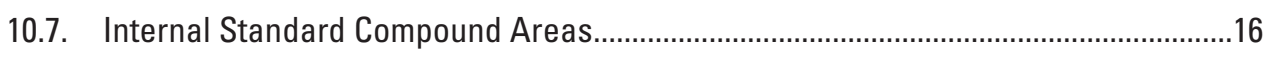

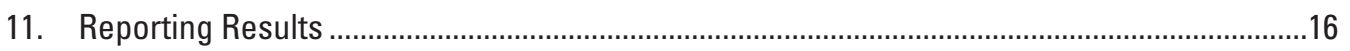


12. Results and Discussion of Method Development and Validation Data ..................................17

12.1. Method Detection Limits and Reporting Levels.....................................................18

12.1.1. Method Detection Limits and Reporting Levels for Method 0-4437 ..............18

12.1.2. Blank-Limited Compounds and Minimum Reporting Levels, Method 4437.........................................................................................18

12.1.3. Discussion of Selected Heat Purgeable Volatile Organic Compounds.........18

12.1.4. Method Detection Limits and Reporting Levels for 0-4436 ...........................19

12.1.5. Blank-Limited Compounds and Minimum Reporting Levels,

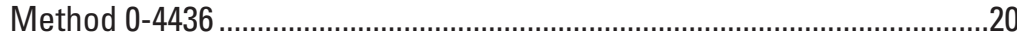

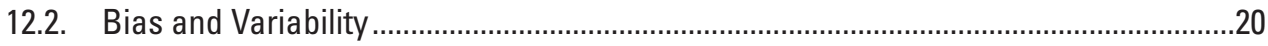

12.2.1. Result of Bias and Variability for Method 0-4437 .......................................20

12.2.2. Results of Bias and Variability for Method 0-4436 .......................................20

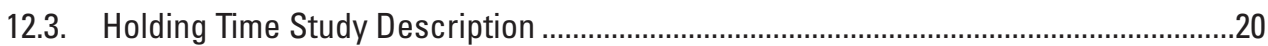

12.3.1. Discussion and Results of Holding Time Study, Method 0-4437 ..................21

12.3.2. Discussion and Results of Holding Time Study, Method 0-4436 ...................21

12.4. Results and Discussion of Laboratory and Environmental Samples

Determined by Method 0-4437 .............................................................................38

12.4.1. Laboratory Continuing Calibration Verification Standards..............................38

12.4.2. Laboratory Reagent-Water Blanks................................................................38

12.4.3. Field-Submitted Blanks ............................................................................38

12.4.4. Environmental Samples..............................................................................39

12.4.5. Laboratory Matrix Spikes.............................................................................39

12.5. Discussion of Heat Purgeable Method 0-4437 and Historical Methods .......................39

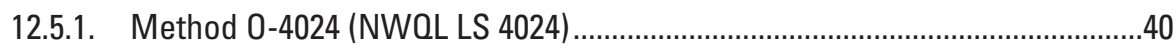

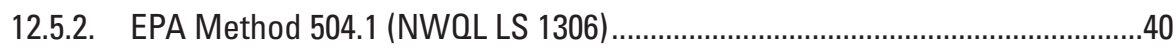

12.5.3. Method 0-4127 (NWOL LS 2020) ..............................................................40

12.5.4. Comparison of Environmental Samples Determined by Heated Purge Selected Ion Monitoring Mass Spectrometry to Ambient Purge

Full Scan Mass Spectrometry for the Fumigant Compounds .................40

12.6. Results and Discussion of Laboratory and Environmental Samples Determined

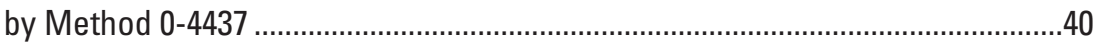

12.6.1. Laboratory Continuing Calibration Verification Standards .................................40

12.6.2. Laboratory Reagent-Water Blanks................................................................

12.6.3. Field-Submitted Blanks..............................................................................

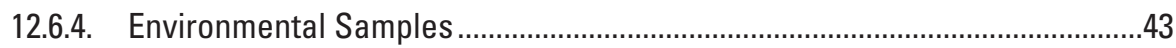

12.6.5. Laboratory Matrix Spikes .........................................................................43

12.7. Discussion of Method 0-4436 (NWQL LS 4436) and Method 0-4127

(NWQL LS 2020) .........................................................................................4

12.7.1. Statistical Comparison of Continuing Calibration Verification Standards Determined by 0-4436 (NWQL LS 4436) and 0-4127 (NWQL LS 2020)......... 44

12.7.2. Comparison of Paired Environmental Samples Determined by 0-4436 (NWQL LS 4436) and 0-4127 (NWQL LS 2020) ........................................51

13. Summary and Conclusions ..............................................................................................52

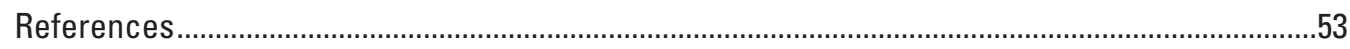

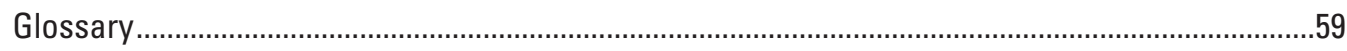

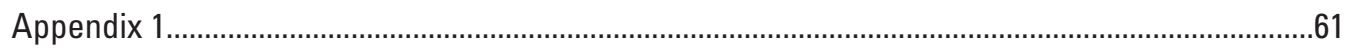




\section{Figures}

1. Selected ion chromatogram of a calibration standard for heat purgeable volatile organic compounds.

2. Total ion chromatogram of a calibration standard for ambient purgeable volatile organic compounds

3-7. Graphs showing:

3. Water solubility compared to Henry's Law Constant for priority compounds selected for testing using purge-and-trap technology......

4. Comparison of method detection limits for ambient purgeable Methods 0-4436 and 0-4127.

5. Summary of a 28-day holding time study for heat purgeable volatile organic compounds .

6. Summary of a 28-day holding time study for ambient purgeable volatile organic compounds...

7. Plot of paired environmental samples determined using both the heated purge method compared to the ambient purge method is shown

8. Boxplots showing comparison of continuing calibration verification standard recoveries for ambient purgeable volatile organic compounds determined by Methods 0-4127 ( $\mathrm{N}=229)$ and 0-4436 ( $\mathrm{N}=245)$, from January 2013 through June 2014

9. Boxplot showing concentrations of volatile organic compounds determined in paired environmental samples determined by ambient purge Method 0-4436 and calculated with 1,2-dichloroethane- $d_{4}$ as the internal standard and by ambient purge Method 0-4127 and calculated with fluorobenzene as the internal standard.

\section{Tables}

1. Heat purgeable volatile organic compounds tested for percent recovery and variability determined by Method 0-4437, simultaneous full scan/selected ion monitoring mass spectrometry.

2. Ambient purgeable volatile organic compounds, tested for percent recovery and variability, determined by Method 0-4436, full scan mass spectrometry.

3. Suggested concentrations of heat purgeable volatile organic compounds in the primary and secondary calibration standards, the calibration range in water, and the concentration of surrogate and internal standard compounds for Method 0-4437, grouped by primary calibration standard mix Link

4. Suggested concentration of ambient purgeable volatile organic compounds in the primary calibration standards, the calibration range in water, and the concentration of the surrogate and internal standard compounds for Method 0-4436

5. Summary of suggested purge-and-trap concentrator, gas chromatograph, and mass spectrometer operating conditions for heat purgeable volatile organic compounds, Method 0-4437 
6. Internal standard compound assignment, quantitation ion, and primary and secondary qualifying ions for heat purgeable volatile organic compounds in Method 0-4437, listed in chromatographic retention time order. .. Link

7. Summary of suggested purge-and-trap concentrator, gas chromatograph, and mass spectrometer operating conditions for ambient purgeable volatile organic compounds for Method 0-4436 . Link

8. Internal standard compound assignment, quantitation ion, and primary and secondary qualifying ions for ambient purgeable volatile organic compounds for Method 0-4436, listed in chromatographic retention time order

9. Tuning performance criteria for mass spectrometer using 1-bromo-4fluorobenzene. .. Link

10. Suggested instrument batch run sequence ............................................................. Link

11. Method detection limits and reporting levels for heat purgeable volatile organic compounds, Method 0-4437 . Link

12. Method detection limits and reporting levels for ambient purgeable volatile organic compounds, Method 0-4436 . Link

13. Percent recovery and variability for heat purgeable volatile organic compounds in volatile blank water, groundwater, and surface water for seven replicates, spiked at two concentrations, and preserved to $\mathrm{pH} 2$ with hydrochloric acid (1:1), Method 0-4437

14. Percent recovery and variability for ambient purgeable volatile organic compounds in volatile blank water, groundwater, and surface water for seven replicates, spiked at two concentrations, and preserved to $\mathrm{pH} 2$ with hydrochloric acid (1:1), Method 0-4436

15. Percent recovery and variability for a 28-day holding time study for heat purgeable volatile organic compounds in volatile blank water, adjusted to $\mathrm{pH} 2$ with hydrochloric acid (1:1), and stored at 4 plus or minus 2 degrees Celsius, Method 0-4437.

16. Summary of parameters used to calculate holding times from a 28-day study for heat purgeable volatile organic compounds in volatile blank water, preserved to $\mathrm{pH} 2$ with hydrochloric acid (1:1), and stored at 4 plus or minus 2 degrees Celsius, using American Society for Testing and Materials procedure D4841-88, Method 0-4437 . Link

17. Percent recovery and variability for a 28-day holding time study for ambient purgeable volatile organic compounds in volatile blank water, adjusted to $\mathrm{pH} 2$ with hydrochloric acid (1:1), and stored at 4 plus or minus 2 degrees Celsius, Method 0-4436 Link

18. Summary of parameters used to calculate holding times from a 28-day study for ambient purgeable volatile organic compounds in volatile blank water, preserved to $\mathrm{pH} 2$ with hydrochloric acid (1:1), and stored at 4 plus or minus 2 degrees Celsius, using American Society for Testing and Materials procedure D4841-88, Method 0-4436 Link

19. Percent recovery and variability of heat purgeable, volatile organic compounds from 75 continuing calibration verification standards, spiked in volatile blank water, preserved to $\mathrm{pH} 2$ with hydrochloric acid (1:1), and determined from January 2013 through August 2013, Method 0-4437 
20. Summary of heat purgeable volatile organic compounds detected in laboratory reagent-water blanks determined from January through

August 2013, Method 0-4437 Link

21. Summary of heat purgeable volatile organic compounds detected in 86 field-submitted blanks determined from January through August 2013, Method 0-4437. Link

22. Summary of environmental sample analyses in groundwater and surface water for heat purgeable volatile organic compounds from January through August 2013, Method 0-4437. Link

23. Percent recovery and variability of heat purgeable volatile organic compounds for 30 laboratory groundwater and surface water matrix spikes, Method 0-4437 . Link

24. Summary of National Water Quality Laboratory schedules and analytical methods for volatile organic compounds Link

25. Summary of paired environmental sample determinations for four fumigant compounds using the heated purge method compared to the ambient purge method Link

26. Percent recovery and variability of ambient purgeable volatile organic compounds for continuing calibration verification standards, spiked at 0.5 micrograms per liter and determined by Method 0-4436. Link

27. Summary of ambient purgeable volatile organic compound detections in laboratory reagent-water blanks determined from January through August 2013, Method 0-4436

28. Summary of ambient purgeable volatile organic compound detections in field-submitted blanks determined from January through August 2013, Method 0-4436 Link

29. Summary of environmental sample analyses in groundwater and surface water for ambient purgeable volatile organic compounds determined from January through August 2013, Method 0-4436. Link

30. Percent recovery and variability of ambient purgeable volatile organic compounds for 28 laboratory groundwater and surface water matrix spikes, preserved in the field to $\mathrm{pH} 2$ with hydrochloric acid (1:1), Method 0-4436. Link

31. Wilcoxon rank sum test of whether the median recoveries in ambient purgeable volatile organic compounds in continuing calibration verification standards determined by Method 0-4436 are the same as those determined by Method 0-4127. Link

32. Levene test and F-test of whether the variability of recoveries of ambient purgeable volatile organic compounds in continuing calibration verification standards determined by Method 0-4436 are the same as those determined by Method 0-4127 Link

\section{Appendix 1}

1-1. Volatile organic compounds discontinued from the ambient purgeable method for routine determination for the National Water-Quality Assessment Program Link

1-2. Physical properties of compounds ranked as priority compounds by the National Water-Quality Assessment Program for purgeable analytical methods Link 


\section{Conversion Factors}

SI to Inch/Pound

\begin{tabular}{|c|c|c|}
\hline Multiply & By & To obtain \\
\hline \multicolumn{3}{|c|}{ Length } \\
\hline micrometer $(\mu \mathrm{m})$ & 0.00003937 & inch (in.) \\
\hline millimeter (mm) & 0.03937 & inch (in.) \\
\hline meter $(\mathrm{m})$ & 3.281 & foot $(\mathrm{ft})$ \\
\hline \multicolumn{3}{|c|}{ Volume } \\
\hline liter (L) & 0.2642 & gallon (gal) \\
\hline milliliter (mL) & 0.000264 & gallon (gal) \\
\hline microliter $(\mu \mathrm{L})$ & 0.000000264 & gallon (gal) \\
\hline \multicolumn{3}{|c|}{ Mass } \\
\hline gram $(\mathrm{g})$ & 0.03527 & ounce, avoirdupois (oz) \\
\hline microgram $(\mu \mathrm{g})$ & 0.00000003527 & ounce, avoirdupois (oz) \\
\hline milligram (mg) & 0.00003527 & ounce, avoirdupois (oz) \\
\hline nanogram (ng) & 0.00000000003527 & ounce, avoirdupois (oz) \\
\hline \multicolumn{3}{|c|}{ Pressure } \\
\hline kilopascal $(\mathrm{kPa})$ & 0.1450 & pound per square inch $\left(\mathrm{lb} / \mathrm{in}^{2}\right)$ \\
\hline \multicolumn{3}{|c|}{ Concentration, in water } \\
\hline milligrams per liter $(\mathrm{mg} / \mathrm{L})$ & 1 & parts per million (ppm) \\
\hline micrograms per liter $(\mu \mathrm{g} / \mathrm{L})$ & 1 & parts per billion (ppb) \\
\hline nanograms per liter (ng/L) & 1 & parts per trillion (ppt) \\
\hline
\end{tabular}

Temperature in degrees Celsius $\left({ }^{\circ} \mathrm{C}\right)$ may be converted to degrees Fahrenheit $\left({ }^{\circ} \mathrm{F}\right)$ as follows: ${ }^{\circ} \mathrm{F}=\left(1.8 x^{\circ} \mathrm{C}\right)+32$

Specific conductance is given in microsiemens per centimeter at 25 degrees Celsius $(\mu \mathrm{S} / \mathrm{cm}$ at $\left.25^{\circ} \mathrm{C}\right)$.

Concentrations of chemical constituents in water in this report are given in micrograms per liter $(\mu \mathrm{g} / \mathrm{L})$.

\section{Abbreviated Water-Quality Units and Units of Measure}

$\begin{array}{ll}\mu \mathrm{g} / \mathrm{L} & \text { microgram per liter } \\ \mathrm{mg} / \mathrm{L} & \text { milligram per liter } \\ \mathrm{amu} & \text { atomic mass unit } \\ \text { ASTM } & \text { American Society for Testing and Materials } \\ \text { BFB } & \text { 1-bromo-4-fluorobenzene } \\ \text { CASRN } & \text { Chemical Abstracts Service Registry Number } \\ \text { CCL 3 } & \text { contaminant candidate list 3 } \\ \text { COB } & \text { carryover blank } \\ \text { CCV } & \text { continuing calibration verification standard }\end{array}$




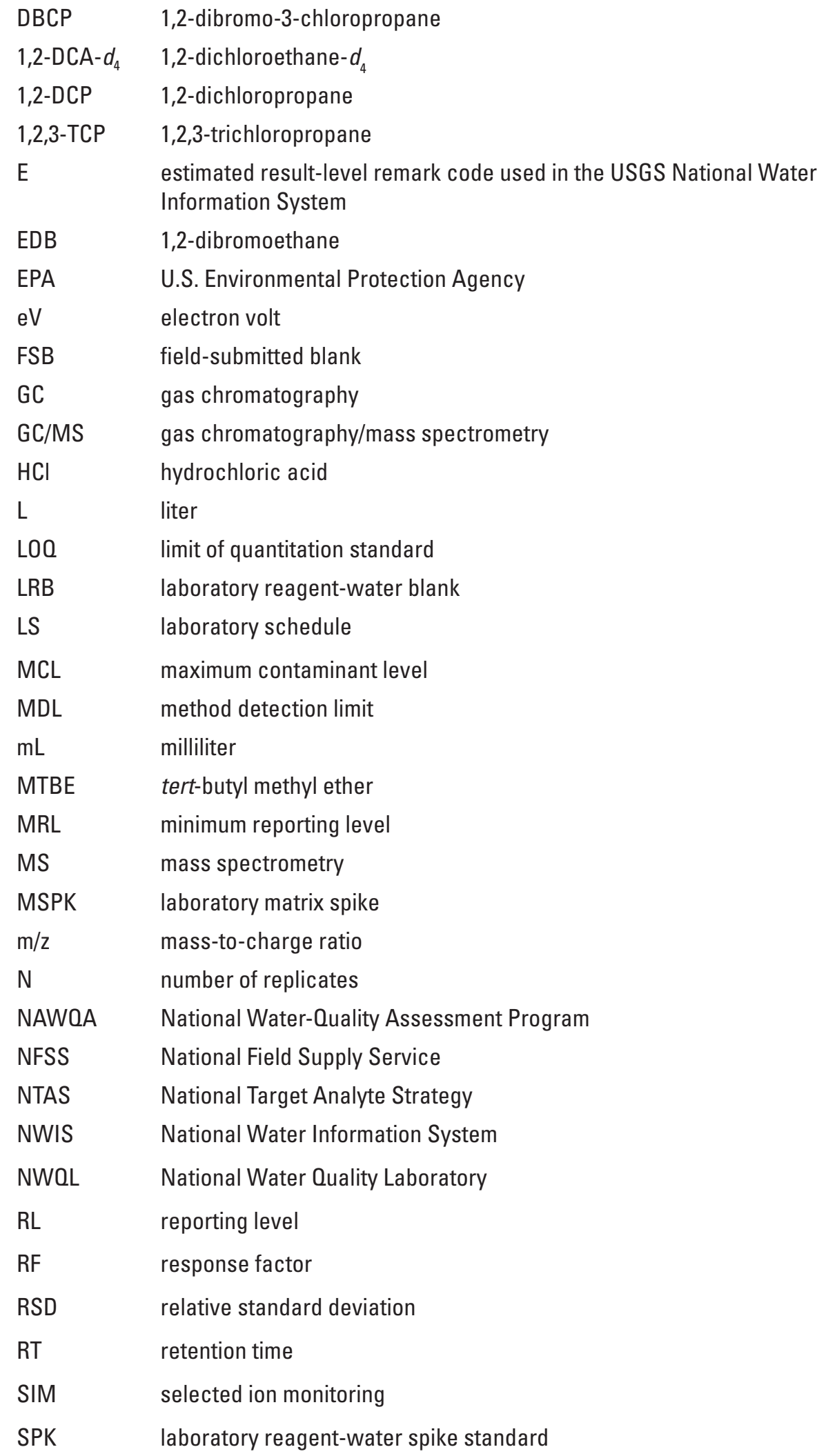




$\begin{array}{ll}\text { UCMR } 3 & \text { unregulated contaminant monitoring rule } 3 \\ \text { USGS } & \text { U.S. Geological Survey } \\ \text { VBW } & \text { volatile blank water } \\ \text { VPBW } & \text { volatile/pesticide-grade water } \\ \text { VOC } & \text { volatile organic compound } \\ = & \text { identical with, congruent } \\ > & \text { greater than } \\ \geq & \text { greater than or equal to } \\ < & \text { less than } \\ \leq & \text { less than or equal to } \\ \pm & \text { plus or minus } \\ \times & \text { times (multiplication) } \\ / & \text { per; divided by } \\ \oplus & \text { registered trademark }\end{array}$





\title{
Determination of Heat Purgeable and Ambient Purgeable Volatile Organic Compounds in Water by Gas Chromatography/ Mass Spectrometry
}

\author{
By Donna L. Rose, Mark W. Sandstrom, and Lucinda K. Murtagh
}

\section{Abstract}

Two new analytical methods have been developed by the U.S. Geological Survey (USGS) National Water Quality Laboratory (NWQL) that allow the determination of 37 heat purgeable volatile organic compounds (VOCs) (USGS Method O-4437-16 [NWQL Laboratory Schedule (LS) 4437]) and 49 ambient purgeable VOCs (USGS Method O-4436-16 [NWQL LS 4436]) in unfiltered water. This report documents the procedures and initial performance of both methods. The compounds chosen for inclusion in the methods were determined as having high priority by the USGS National WaterQuality Assessment (NAWQA) Program. Both methods use a purge-and-trap technique with gas chromatography/mass spectrometry. The compounds are extracted from the sample by bubbling helium through a 25 -milliliter sample. For the polar and less volatile compounds, the sample is heated at 60 degrees Celsius, whereas the less polar and more volatile compounds are purged using a separate analytical procedure at ambient temperature. The compounds are trapped on a sorbent trap, desorbed into a gas chromatograph/mass spectrometer for separation, and then identified and quantified. Sample preservation is recommended for both methods by adding a 1:1 solution of hydrochloric acid ( $\mathrm{HCl}[1: 1])$ to water samples to adjust the $\mathrm{pH}$ to 2 . Analysis within 14 days from sampling is recommended.

The heat purgeable method (USGS Method O-4437-16) operates with the mass spectrometer in the simultaneous full scan/selected ion monitoring mode. This method supersedes USGS Method O-4024-03 (NWQL LS 4024). Method detection limits (MDLs) for fumigant compounds 1,2-dibromoethane, 1,2-dichloropropane, 1,2,3-trichloropropane, chloropicrin, and 1,2-dibromo-3-chloropropane range from 0.002 to 0.010 microgram per liter $(\mu \mathrm{g} / \mathrm{L})$. The MDLs for all remaining heat purgeable VOCs range from $0.006 \mu \mathrm{g} / \mathrm{L}$ for tert-butyl methyl ether to $3 \mu \mathrm{g} / \mathrm{L}$ for alpha-terpineol. Calculated holding times indicate that 36 of the 37 heat purgeable VOCs are stable for a minimum of 14 days preserved with $\mathrm{HCl}(1: 1)$ to $\mathrm{pH}$ 2, and many are stable longer. Acrolein was retained in the method validation and initial method implementation and subsequently deleted because of instability and inconsistent performance. 2-Chloromethyl oxirane, methyl oxirane, and oxirane were tested using this method, but the compounds degraded quickly with the $\mathrm{HCl}(1: 1)$ used for microbial preservation.

The ambient purgeable method, USGS Method O-4436-16, operates with the mass spectrometer in the full scan mode. This method is a modification of USGS Method O-4127-96 (NWQL LS 2020). Several compounds were retained from Method O-4127-96 and will continue to be determined in Method O-4436-16. Eleven high priority compounds were added. MDLs for the high priority compounds range from $0.007 \mu \mathrm{g} / \mathrm{L}$ for 2,2-dichloro-1,1,1-trifluoroethane to $0.04 \mu \mathrm{g} / \mathrm{L}$ for 1,2,3,4-tetrahydronaphthalene and 1,3-butadiene. Historical MDLs for the compounds retained from Method O-4127-96 range from $0.009 \mu \mathrm{g} / \mathrm{L}$ for trans-1,2-dichloroethene to $0.1 \mu \mathrm{g} / \mathrm{L}$ for bromomethane. The calculated holding times for the compounds indicate the majority of the compounds are stable for a minimum of 14 days, or longer, at $\mathrm{pH} 2$ with $\mathrm{HCl}$ (1:1) preservation. Four semivolatile compounds, 1,2-dimethylnaphthalene, 1,6-dimethylnaphthalene, 2,6-di-tert-butyl phenol, and 2-chloronapthalene, were tested and deleted from the method due to poor performance. Benzyl chloride was tested and deleted due to instability.

\section{Introduction}

The U.S. Geological Survey's (USGS) National WaterQuality Assessment (NAWQA) Program assembled a team to review and prioritize emerging contaminants for NAWQA's third decade of assessment, Cycle 3. The National Target Analyte Strategy (NTAS) team reviewed more than 3,000 compounds in water and sediment, and prioritized them into three priority tiers. The compounds reviewed were screened using both occurrence and toxicity data. The compounds were ranked into tiers 1 (highest priority), 2 (intermediate priority), and 3 (lowest priority), and categorized into nine chemical groups, including algal toxins, disinfection byproducts, high production volume chemicals, industrial constituents, volatile organic compounds (VOCs), lipophilic organics, pesticides, pharmaceuticals, and trace elements (Olsen 
and others, 2013). Some of the compounds in tiers 1 and 2 are subject to National Primary Drinking Water Regulations with promulgated maximum contaminant levels (MCLs) by the U.S. Environmental Protection Agency (EPA). Other tier 1 and 2 compounds may not be regulated, but have been identified as priority contaminants in the third EPA Contaminant Candidate List (CCL 3) for possible occurrence monitoring under the EPA Unregulated Contaminant Monitoring Rule (UCMR). Occurrence data for 1,3-butadiene, 1,2,3-trichloropropane, chloromethane, 1,1-dichloroethane, bromomethane, chlorodifluoromethane, and bromochloromethane are being collected under the third UCMR cycle (UCMR 3) (U.S. Environmental Protection Agency, 2012).

As a result of the findings by the NTAS team, analytical methods were developed at the USGS National Water Quality Laboratory (NWQL) for analysis of purgeable VOCs. The 91 VOC compounds ranked in tier 1 were candidates for method development efforts using purge-and-trap analytical methods. Initial evaluation of the tier 1 compounds involved determining which purge temperature, 60 degrees Celsius $\left({ }^{\circ} \mathrm{C}\right)$ or ambient, was suitable. Although most VOCs could be determined using heated purge conditions, only the compounds with poor purge response under ambient purge conditions, and those requiring lower method detection limits (MDLs) were recommended for testing using the heated purge conditions. tert-Butyl methyl ether (MTBE) is the only compound included in both methods.

Major modifications were made to USGS Method O-4024-03 (henceforth referred to as O-4024) (NWQL Laboratory Schedule [LS] 4024) (Rose and Sandstrom, 2003) by implementing simultaneous full scan/selected ion monitoring (SIM) electron impact mass spectrometry, and modifying sample preservation. The 37 tier 1 compounds determined by this method included four fumigant compounds from USGS Method O-4127-96 (henceforth referred to as O-4127) (NWQL LS 2020) (Connor and others, 1998), which required lower MDLs. Minor modifications were made to USGS Method O-4127 (NWQL LS 2020) to accommodate 11 high priority compounds. Twenty-five compounds were not purgeable using either method; 18 compounds were dropped for poor performance or stability issues.

Since 1998, 85 VOCs have been monitored by NAWQA using USGS Method O-4127 (NWQL LS 2020) (Connor and others, 1998). These 85 compounds are referred to as "ambient purgeable volatile organic compounds" in this report. Compounds determined by this method were ranked by the NTAS team in all three tiers. Tier 1 compounds were retained, tier 2 compounds were retained if they appeared on one of EPA's regulated or unregulated compound lists, and tier 3 compounds were discontinued from NAWQA Cycle 3 monitoring and were not considered for the methods.

\section{Scope and Application}

This report documents work at the USGS NWQL to validate determinative methods for unfiltered water for VOCs using purge-and-trap techniques, with gas chromatography and mass spectrometry. In these low-level methods, VOCs are purged from water samples at $60{ }^{\circ} \mathrm{C}$ (USGS Method O-4437-16 [henceforth referred to as Method 0-4437] [NWQL LS 4437], heat purgeable VOCs) or at ambient temperature (USGS Method O-4436-16 [henceforth referred to as Method 0-4436] [NWQL LS 4436], ambient purgeable VOCs). The methods are suitable for the determination of VOCs at low microgram per liter $(\mu \mathrm{g} / \mathrm{L})$ to nanogram per liter concentrations. The methods are applicable to groundwater, surface water, and treated water. Refer to table 1 for heat purgeable VOCs (USGS Method O-4437 [NWQL LS 4437]) and table 2 for ambient purgeable VOCs (USGS Method O-4436 [NWQL LS 4436]). Refer to the Appendix table 1-1 for the list of VOCs that were discontinued for NAWQA Cycle 3.

The report outlines all procedures for Method O-4437 (NWQL LS 4437) and Method O-4436 (NWQL LS 4436), including sample collection and preservation, sample preparation, reagents, standard preparation, instrumentation, calibration, instrumental analysis, interferences, identification and quantitation, quality control (QC) requirements, reporting requirements, and safety and waste disposal. Method validation results, including bias and variability, MDLs, and the holding time study are discussed. Preliminary implementation and testing of both methods include results of laboratory QC samples, environmental sample determinations, fieldsubmitted QC samples, and results of paired environmental sample determinations.

\section{Method Summary}

In both the heated and ambient purgeable methods, VOCs are purged from the sample matrix by bubbling helium through a 25 -milliliter $(\mathrm{mL})$ aqueous sample, at either $60^{\circ} \mathrm{C}$ or ambient temperature. The compounds are trapped in a tube containing suitable sorbent materials, and then thermally desorbed into a capillary gas chromatographic column (GC) interfaced to a mass spectrometer (MS). For the heat purgeable method, the compounds are determined using electron impact (EI) ionization mass spectrometry in the simultaneous full scan/selected ion monitoring (SIM) mode. The heat purgeable method reports data from the SIM data file, using the full scan data file for confirmation if interferences are present from nontarget compounds. For the ambient purgeable method, a modification of USGS Method O-4127 (NWQL LS 2020), the compounds are determined using EI ionization mass spectrometry in the full scan mode. In both methods compounds are identified using strict identification criteria, which include analyzing standard reference materials, comparing retention times, and relative ion ratios in each mass spectrum. Compounds are quantitated using internal standard procedures. Samples with concentrations above the highest calibration standard are diluted to within the calibration range and reanalyzed. An electronic version of the standard operating procedure for the methods is available upon request to LabHelp@usgs.gov. 
Table 1. Heat purgeable volatile organic compounds tested for percent recovery and variability determined by Method 0-4437, simultaneous full scan/selected ion monitoring mass spectrometry.

[Validation quality code: A, acceptable, no remark code qualification; E, estimated remark code; D, deleted from the method; CASRN, Chemical Abstracts Service Registry Number; NTAS, National Target Analyte Strategy; --, not applicable]

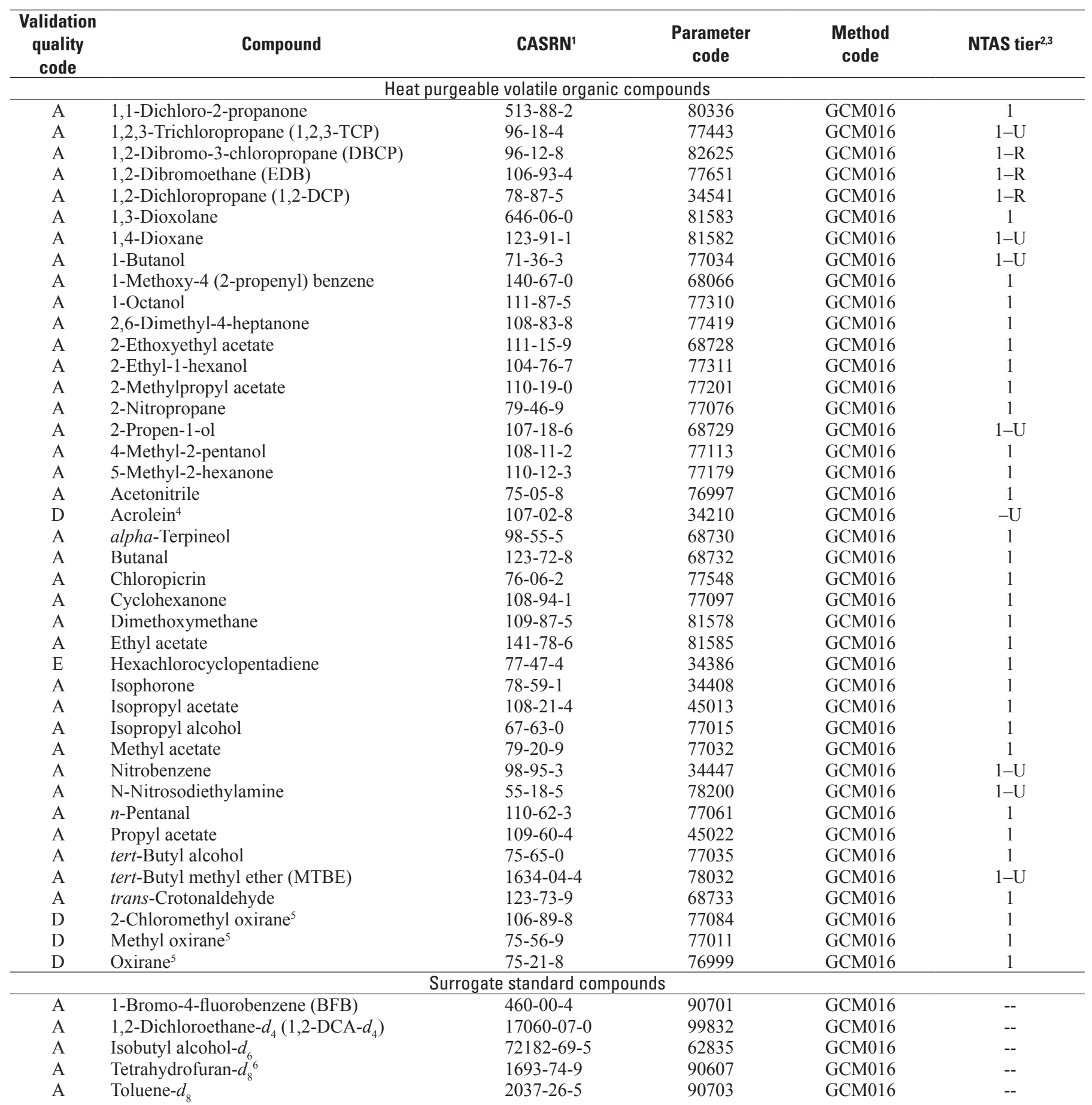


Table 1. Heat purgeable volatile organic compounds tested for percent recovery and variability determined by Method 0-4437, simultaneous full scan/selected ion monitoring mass spectrometry.-Continued

[Validation quality code: A, acceptable, no remark code qualification; E, estimated remark code; D, deleted from the method; CASRN, Chemical Abstracts Service Registry Number; NTAS, National Target Analyte Strategy; --, not applicable]

\begin{tabular}{|c|c|c|c|c|c|}
\hline $\begin{array}{c}\text { Validation } \\
\text { quality } \\
\text { code }\end{array}$ & Compound & CASRN ${ }^{1}$ & $\begin{array}{l}\text { Parameter } \\
\text { code }\end{array}$ & $\begin{array}{l}\text { Method } \\
\text { code }\end{array}$ & NTAS tier ${ }^{2,3}$ \\
\hline \multicolumn{6}{|c|}{ Internal standard compounds } \\
\hline-- & 1,4 -Dioxane- $d_{8}$ & $17647-74-4$ & -- & GCM016 & -- \\
\hline-- & Fluorobenzene & $462-06-6$ & -- & GCM016 & -- \\
\hline-- & tert-Butyl alcohol- $d_{10}$ & $53001-22-2$ & -- & GCM016 & -- \\
\hline
\end{tabular}

${ }^{1}$ This report contains CAS Registry Numbers ${ }^{\circledR}$, which is a Registered Trademark of the American Chemical Society. CAS recommends the verification of the CASRNs through CAS Client ServicesSM.

${ }^{2}$ For more information on NTAS tier ranking, refer to Olsen and others, 2013.

3"-R" indicates a regulated U.S. Environmental Protection Agency (EPA) drinking water compound; "-U" indicates an unregulated compound on the EPA Contaminant Candidate List 3 (CCL 3) and (or) EPA Unregulated Contaminant Monitoring Rule 3 (UCMR 3).

${ }^{4}$ Acrolein was not ranked by the NTAS team. It was added because it is included on the EPA CCL 3 list. Acrolein was retained on Method O-4437 from January 2013 through February 2016. After reviewing the data collected, the recommendation was made to remove acrolein from Method O-4437 due to inconsistent performance, instability, and issues obtaining reference standards. Data determined for acrolein by Method O-4437 are included for documentation.

${ }^{5}$ These compounds were incompatible with the hydrochloric acid (1:1) preservative and were not recommended for inclusion in this method. Available data collected during method development are included for documentation.

${ }^{6}$ This surrogate was added during method validation in January 2013, after the initial method development was completed.

Table 2. Ambient purgeable volatile organic compounds, tested for percent recovery and variability, determined by Method 0-4436, full scan mass spectrometry.

[Validation quality code: A, acceptable, no remark code qualification; E, estimated remark code; D, deleted from method; CASRN, Chemical Abstracts Service Registry Number; NTAS, National Target Analyte Strategy; --, not applicable]

\begin{tabular}{|c|c|c|c|c|c|}
\hline $\begin{array}{c}\text { Validation } \\
\text { quality } \\
\text { code }\end{array}$ & Compound & CASRN & $\begin{array}{c}\text { Parameter } \\
\text { code }\end{array}$ & $\begin{array}{l}\text { Method } \\
\text { code }\end{array}$ & NTAS tier ${ }^{1,2}$ \\
\hline \multicolumn{6}{|c|}{ High priority ambient purgeable volatile organic compounds } \\
\hline A & $1,2,3,4$-Tetrahydronaphthalene & $119-64-2$ & 77323 & GCM66 & 1 \\
\hline A & 1,2-Dichloro-1,1,2,2-tetrafluoroethane & $76-14-2$ & 50985 & GCM66 & 1 \\
\hline A & 1,3-Butadiene & $106-99-0$ & 68726 & GCM66 & $1-U$ \\
\hline A & Butane & $106-97-8$ & 81563 & GCM66 & 1 \\
\hline A & Chlorodifluoromethane & $75-45-6$ & 45028 & GCM66 & $1-U$ \\
\hline A & Dichlorofluoromethane & $75-43-4$ & 77119 & GCM66 & -- \\
\hline $\mathrm{E}$ & Hexane & $110-54-3$ & 81590 & GCM66 & $1-\mathrm{U}$ \\
\hline A & Pentane & $109-66-0$ & 81604 & GCM66 & 1 \\
\hline $\mathrm{D}$ & 1,2-Dimethylnaphthalene ${ }^{3}$ & $573-98-8$ & 68725 & GCM66 & 1 \\
\hline \multicolumn{6}{|c|}{ Ambient purgeable volatile organic compounds } \\
\hline $\mathrm{A}$ & 1,1,1,2-Tetrachloroethane & $630-20-6$ & 77562 & GCM66 & $2-U$ \\
\hline A & $1,1,1$-Trichloroethane & $71-55-6$ & 34506 & GCM66 & $1-\mathrm{R}$ \\
\hline A & 1,1,2-Trichloroethane & $79-00-5$ & 34511 & GCM66 & $2-\mathrm{R}$ \\
\hline A & 1,1-Dichloroethane & $75-34-3$ & 34496 & GCM66 & $1-\mathrm{U}$ \\
\hline
\end{tabular}


Table 2. Ambient purgeable volatile organic compounds, tested for percent recovery and variability, determined by Method 0-4436, full scan mass spectrometry.-Continued

[Validation quality code: A, acceptable, no remark code qualification; E, estimated remark code; D, deleted from method; CASRN, Chemical Abstracts Service Registry Number; NTAS, National Target Analyte Strategy; --, not applicable]

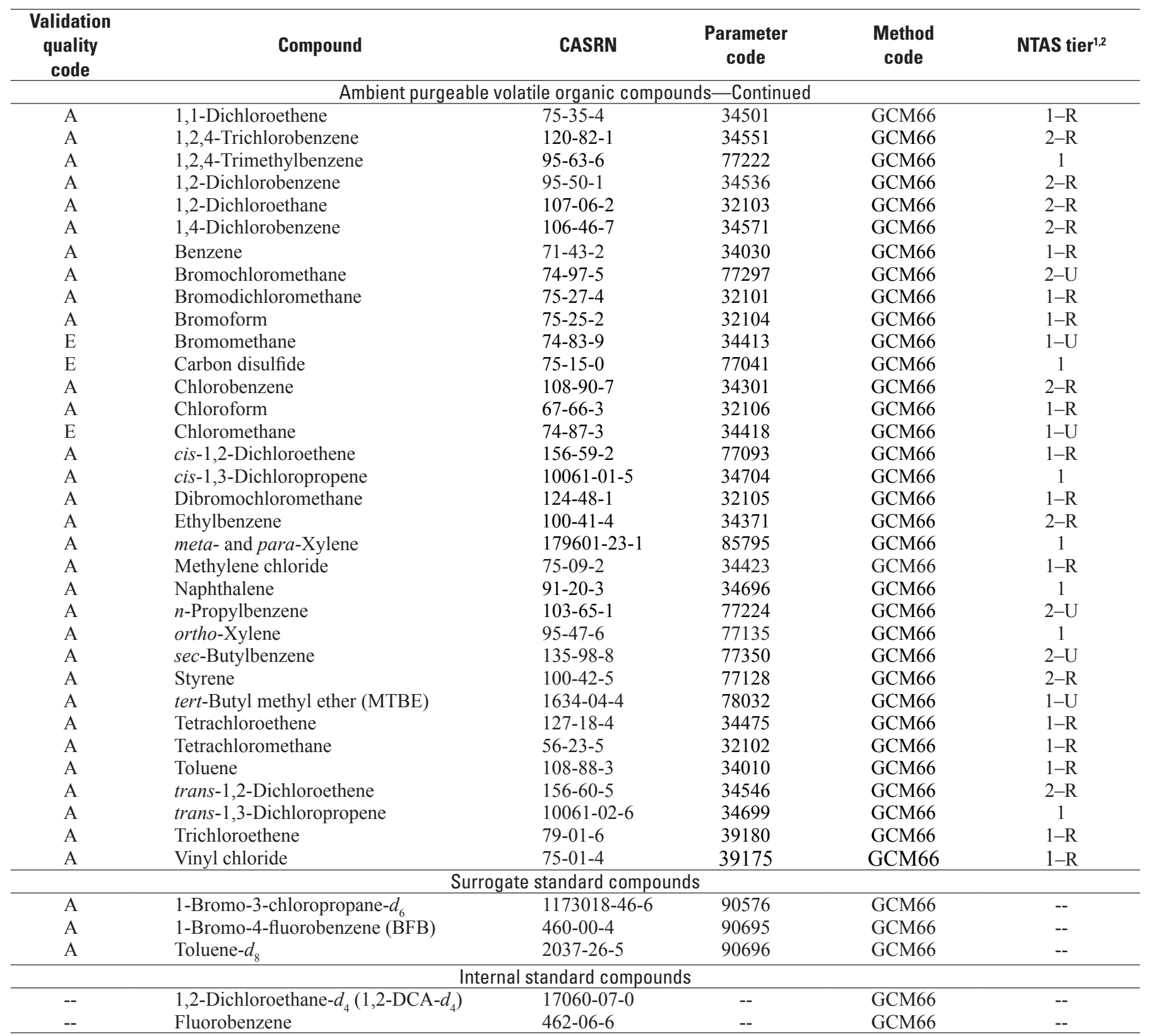

${ }^{1}$ For more information on NTAS Tier ranking, refer to Olsen and others, 2013.

2"-R" indicates a regulated U.S. Environmental Protection Agency (EPA) drinking water compound; "-U" indicates an unregulated compound on the EPA Contaminant Candidate List 3 (CCL 3) and (or) EPA Unregulated Contaminant Monitoring Rule 3 (UCMR 3).

${ }^{3}$ These compounds were removed from the method because of poor performance. Available data acquired during method development are provided for documentation.

${ }^{4}$ Benzyl chloride was not ranked by the NTAS team. It was tested because it is included on the USEPA CCL 3 list. Benzyl chloride was not recommended for inclusion in the method because of instability and poor performance. Available data acquired during method development are provided for documentation. 


\section{Safety Precautions and Waste Disposal}

The methods described herein use solvents, reagents, and chemicals that possess risks to human health. These methods should only be undertaken with the knowledge of the risks involved and the use of protective equipment and engineering controls. Obtain copies of safety data sheets for the relevant reagents and compounds from the manufacturers. Other sources of chemical safety are available online from the U.S. National Library of Medicine TOXNET toxicological data network (U.S. National Library of Medicine, 2015), and the National Institute of Occupational Safety and Health website (Centers for Disease Control and Prevention, National Institute of Occupational Health and Safety, 2015). At a minimum, personnel should wear a laboratory coat, safety glasses, and nitrile gloves when working with these chemicals and employ fumecontainment controls as appropriate. Consult with the unit supervisor, safety personnel, or other experienced person about how to perform the methods safely.

Potential exposure to high voltage electrical current exists when servicing equipment used in these methods. Make sure to disconnect equipment from the power source when servicing the unit. Precautions should be taken when performing maintenance on the gas chromatograph/mass spectrometer and the purge-andtrap concentrator. Heated zones should be cooled prior to any maintenance. Temperatures can be as high as $300^{\circ} \mathrm{C}$.

Samples for these methods may be from sites characterized as biohazardous. If samples are potentially biohazardous, they must be labeled as "biohazardous" and be handled according to the specific requirements for treatment and disposal. Wilde and others (2004, chapter A5) provides guidelines for collection and processing of biohazardous samples. Unpublished internal NWQL standard operating procedure MULX0430.1, Handling of potential biohazardous samples, (D.L. Damrau, and H.L. Wharry, U.S. Geological Survey, written commun., 2014), provides details of handling biohazardous samples in the laboratory.

Disposal of materials must be carried out in strict accordance with local regulations. All reference standards and solvents must be disposed of as organic waste. Acidified aqueous waste from the instrument must be contained in carboys and disposed of according to local regulations.

\section{Interferences}

Samples can become contaminated during collection. Strict $\mathrm{QC}$ is required to maintain cleanliness at the sampling site. Field equipment blanks are intended to provide $\mathrm{QC}$ on possible sources of field contamination. Refer to Thiros and others (2011) for an in-depth discussion on minimizing sources of external contamination. Refer to Chapter A1 in the USGS National Field Manual for sample collection procedures (Wilde and others, 1999).
Special care is taken to eliminate all potential organic contaminants from the VOC laboratory. Only clothing that has not been exposed to solvent vapors is worn. The analytical laboratory for volatiles is maintained under positive pressure and located far from other laboratories where extractions using organic solvents are performed. To reduce the possibility of contaminating samples, laboratory solvents, with the exception of methanol, are stored outside of the VOC laboratory. Moreover, VOC stock solutions are not stored near samples.

Samples containing high concentrations of VOCs can cause cross contamination during the next determination at detectable concentrations because of residual VOCs in the trap, purge vessel, or transfer lines. Carryover contamination can vary by instrument and by compound, and should be monitored by determination of carryover blanks (COBs), and redetermination of samples following high concentration samples.

Hydrogen sulfide and other forms of sulfur can alter the calibration of some compounds, including bromomethane, chloromethane, and hexachlorocyclopentadiene, requiring recalibration or qualification of results. Indications of the presence of sulfur in water samples include the detection of nontarget compounds, such as sulfur dioxide or carbonyl sulfide, within the first several minutes of the total ion chromatogram, altered calibration response in the following QC samples, or an odor of "rotten egg" when handling the sample. Samples with high alkalinity and (or) a high specific conductivity may require dilution. Such samples include oil field brines, hydraulic fracturing fluids/wastewaters, and landfill leachates.

\section{Supplies and Instrumentation}

Laboratory supplies, sample collection supplies, and instrumentation used for the methods are outlined.

\subsection{Laboratory Supplies}

5.1.1. GC Analytical Columns-Restek ${ }^{\circledR} \mathrm{Rtx}-\mathrm{VGC} 60$-meter (m) $\times 0.25$-millimeter $(\mathrm{mm})$ inner diameter (ID), 1.4-micrometer $(\mu \mathrm{m})$ film thickness, or Restek ${ }^{\circledR}$ Rtx-624 60-m × 0.25-mm ID, 1.4- $\mu \mathrm{m}$ film thickness, or equivalent.

5.1.2. Analytical Purge Traps-OI Corporation ${ }^{\circledR}$ number 7 (tenax) for heat purgeable method, Tekmar $^{\mathbb{B}} \mathrm{K}$ $\left(\right.$ VOCARB $\left.^{\circledR} 3000\right)$ for ambient purgeable method, or equivalent.

5.1.3. Glassware - Class A volumetric glassware, $1-100 \mathrm{~mL}$, baked at $120^{\circ} \mathrm{C}$ for at least 1 hour (hr), 4-L Pyrex flasks with boiling chips for preparing volatile blank water.

5.1.4. Syringes - Glass microsyringes delivering $1 \mu \mathrm{L}$ to $1 \mathrm{~mL}$, glass syringes delivering $1-50 \mathrm{~mL}$, with stainless steel needles. 
5.1.5. Hot Plate-One or two hot plates fitted with an external timer and capable of boiling water in a 4-L Pyrex glass flask.

5.1.6. VOC Vials-Amber $40-\mathrm{mL}$ borosilicate vials, with Teflon-faced septa, C\&G Scientific Containers, catalog number LW74-A040-A01G, or equivalent.

\subsection{Sample Collection Supplies}

The following supplies are quality-assured by the NWQL and available for purchase from the National Field Supply Service (NFSS; http://nwql.usgs.gov/about-contacts.shtml, NWQL, Denver, Colo.).

5.2.1. Hydrochloric Acid Preservative ( $\mathrm{HCl}[1: 1])-1: 1$ solution of $\mathrm{HCl}$ and volatile blank water; packaged in a 30-mL Teflon ${ }^{\circledR}$ dropper bottle. Do not use stock $\mathrm{HCl}$ purchased in high density polyethylene bottles because of possible contamination with tert-butyl alcohol.

(NFSS catalog number N1140).

5.2.2. Trip Blanks_-Prepared by filling a VOC vial with volatile blank water; prepared on demand (NFSS catalog number N1540).

5.2.3. VOC Vials-Amber $40-\mathrm{mL}$ borosilicate vials, with Teflon $^{\circledR}$-faced septa, C\&G Scientific Containers, catalog number LW74-A040-A01G, or equivalent (NFSS catalog number N1560).

5.2.4. VOC Vials with Dechlorination Reagent-Amber 40-mL borosilicate vials, with Teflon ${ }^{\circledR}$-faced septa, with $4 \mathrm{mg}$ ascorbic acid added as a dechlorination reagent; prepared on demand (NFSS catalog number N1160).

5.2.5. Water, Nitrogen Purged, Organic Blank_-VOC/pesticide grade (VPBW). Commercial blank water (EMD Chemicals, catalog number WX0004-1, OmniSolv ${ }^{\circledR}$ for high pressure liquid chromatography [HPLC], or equivalent) is purged with ultra-high purity nitrogen for 2 hours to remove trace volatiles before recapping and shipping to USGS Water Science Centers. After purging, VPBW has a shelf life of 2 weeks (NFSS catalog number N1580). VPBW is used for equipment rinsing, source solution blanks, and field equipment blanks.

\subsection{Instrumentation}

5.3.1. Autosampler-OI Corporation ${ }^{\circledR}$ Model 4552, Tekmar Solatek automatic vial autosampler, or equivalent. The autosamplers are equipped to hold 40-mL VOC vials, transfer up to $25 \mathrm{~mL}$ of sample to the purge vessel, and chill samples to $4{ }^{\circ} \mathrm{C} \pm 2{ }^{\circ} \mathrm{C}$.
5.3.2. Purge-and-Trap Sample Concentrator - OI Corporation ${ }^{\circledR}$ Eclipse $^{\circledR}$ Model 4660 purge-and-trap sample concentrator, Tekmar Velocity ${ }^{\circledR}$ concentrator, or equivalent; capable of heating a $25-\mathrm{mL}$ sample using an infrared heater, exterior heating jacket, or equivalent.

5.3.3. Gas Chromatograph-Hewlett-Packard Model 6890 or equivalent gas chromatograph, equipped with electronic pressure control; configured in the split/splitless mode.

5.3.4. Mass Spectrometer-Hewlett-Packard Model 5973 or 5975 mass selective detector or equivalent, capable of analyzing data in the simultaneous full scan/SIM mode using EI ionization.

5.3.5. Software-Data acquisition, Agilent MSD ChemStation, revision E.02.02, or equivalent; data reduction, ThermoQuest Thru-Put software, Target Chromatographic Analysis Software, revision 4.14, or equivalent.

\section{Reagents}

6.1. Helium (He) - Chemical Abstracts Service Registry Number (CASRN) 7440-59-7; gas chromatograph carrier gas and purge gas, ultra high purity, 99.999 percent, obtain from local supplier.

6.2. Hydrochloric Acid ( $\mathrm{HCl}$ - C CASRN 7647-01-0; preservative, Fisher Optima $\mathrm{HCl}$, catalog number A466-250 or equivalent, packaged in specially manufactured perfluoroalkoxy fluoropolymer bottles. $\mathrm{HCl}$ may also be purchased in glass or Teflon ${ }^{\circledR}$ bottles. $\mathrm{HCl}$ purchased in high density polyethylene bottles has shown contamination for tert-butyl alcohol.

6.3. Methanol $\left(\mathrm{CH}_{3} \mathrm{OH}\right)$ - CASRN 67-56-1; purge-and-trap grade, Burdick \& Jackson ${ }^{\circledR}$ for purge-and-trap analysis, catalog number $232-235$ or equivalent.

6.4. Nitrogen Gas ( $N_{2}$ ) — CASRN 7727-37-9; ultra high purity, delivered by an in-house bulk tank from a local supplier, with stainless steel plumbing (1/8 inch) to a 4-L separatory funnel in the VOC laboratory containing volatile blank water, providing continuous purging.

6.5. Water $\left(\mathrm{H}_{2} \mathrm{O}\right)$, Volatile Blank $(\mathrm{VBW})$ - CASRN 7732-18-5; deionized or distilled in glass, prepared onsite, boiled for $1 \mathrm{hr}$, cooled, and purged with ultrahigh purity nitrogen continuously, for a minimum of $1 \mathrm{hr}$. After boiling and purging, VBW should not contain measurable amounts of method compounds or interferences greater than the MDL for each compound. 
VBW is prepared daily in the laboratory to use for preparing standards and laboratory reagent-water blanks. Deionized boiled water is used for the rinse water reservoirs on the autosamplers.

6.6. Ascorbic Acid $\left(\mathrm{C}_{6} \mathrm{H}_{8} \mathrm{O}_{6}\right)$ - CASRN 50-81-7; dechlorinating agent, Sigma-Aldrich, L-ascorbic acid, 99+ percent, catalog number 255564-100G, or equivalent. Ascorbic acid is required when free chlorine is present in water samples.

\section{Standards}

Concentrated methanol, methanol/water, or water solutions of the compounds of interest are described. All methanol and methanol/water solutions are stored with minimum headspace in a freezer at $-10{ }^{\circ} \mathrm{C}$ or colder. Solutions prepared in water are stored in a refrigerator at $4{ }^{\circ} \mathrm{C} \pm 2{ }^{\circ} \mathrm{C}$. VOC solutions sealed in glass ampoules, and stored as stated, are stable for up to 3 years, depending on the compound. Once opened, the solutions are transferred to $1-\mathrm{mL}$ or 2-mL hole-cap screw vials with Teflon liners. Depending on the contents, solutions refrigerated in hole-cap screw vials may remain stable for 2-6 months. All standard solutions are stored separately in freezers or refrigerators that do not contain samples. All standard solutions are prepared using purge-and-trap grade methanol or deionized water.

\subsection{Mass Spectrometer Performance Evaluation Standard Solution}

Prepare a 25 microgram per milliliter $(\mu \mathrm{g} / \mathrm{mL})$ solution of 1-bromo-4-fluorobenzene (BFB) in purge-and-trap grade methanol. Alternatively, mass spectrometer performance may be evaluated from the surrogate/internal standard solution, which includes BFB.

\subsection{Surrogate and Internal Standard Compound Solution, Method 0-4437}

Obtain commercially prepared standards or prepare the internal standard and surrogate compounds listed in table 3 from individual neat standards, with concentrations ranging from 1,000 to $10,000 \mu \mathrm{g} / \mathrm{mL}$ in methanol. Neat standards were obtained from Supelco, Inc. (Bellefonte, Penn.) and CDN Isotopes (Canada). Prepare the solution so when the autosampler injects it into the $25-\mathrm{mL}$ water sample, the final desired concentration is $1 \mu \mathrm{g} / \mathrm{L}$ for 1-bromo-3-chloropropane- $d_{6}$, 1,2-dichloroethane- $d_{4}$ $\left(1,2-\mathrm{DCA}-d_{4}\right), \mathrm{BFB}$, fluorobenzene, and toluene- $d_{8} ; 2 \mu \mathrm{g} / \mathrm{L}$ for tetrahydrofuran- $d_{8}$, and $10 \mu \mathrm{g} / \mathrm{L}$ for isobutyl alcohol- $d_{6}$, tert-butyl alcohol- $d_{10}$, and 1,4-dioxane- $d_{8}$. Pour the solution into the standard addition vessel on the autosampler.

\subsection{Surrogate and Internal Standard Compound Solution, Method 0-4436}

Obtain commercially prepared standards, or prepare the internal standard and surrogate compounds listed in table 4 from individual neat standards at $5,000 \mu \mathrm{g} / \mathrm{mL}$ in methanol. Neat standards were obtained from Supelco, Inc. (Bellefonte, Penn.) and CDN Isotopes (Canada). Prepare the solution so when the autosampler injects it into the $25-\mathrm{mL}$ water sample, the final desired concentration is $1 \mu \mathrm{g} / \mathrm{L}$ for each compound. Pour the solution into the standard addition vessel on the autosampler.

\subsection{Calibration Standard Solutions, Method 0-4437}

Obtain commercially prepared primary calibration standards in methanol, water, or a methanol/water mix for the compounds listed in table 3 . Because of the wide range of response factors for the compounds in this method, the concentration of the primary calibration standard is optimized for each compound, ranging from $100 \mu \mathrm{g} / \mathrm{mL}$ to $20,000 \mu \mathrm{g} / \mathrm{mL}$. Alternatively, prepare from individual neat standards. The composition and number of the separate primary calibration standards are determined by shelf-life limitations, compound class, or commercially available mixes. The compounds are divided into six primary calibration standards. Group the volatile organic esters (methyl acetate, ethyl acetate, propyl acetate, isopropyl acetate, 2-methylpropyl acetate, and 2-ethoxyethyl acetate) into one primary calibration standard (mix 5, table 3). Methanol solutions containing halogenated compounds are slightly acidic, allowing the esters to react with alcohols to make new esters. An increasing concentration of methyl acetate over time was observed during method development when primary calibration standard mix 1 contained the halogenated compounds and the volatile organic esters. Due to its shelf-life of 4-6 months, acrolein (mix 4, table 3 ) was obtained as a commercially prepared individual standard. Refer to table 3 for the concentrations, solvents, groupings, and calibration ranges used in this method.

Prepare two secondary calibration standard solutions, in purge-and-trap grade methanol, combining the primary calibration standards, to make one solution containing all compounds for each secondary calibration standard. Prepare the secondary calibration standards so that no more than $25 \mu \mathrm{L}$ is spiked into $50 \mathrm{~mL}$ of water to prepare the calibrations standards.

Monitor the secondary calibration standard frequently by comparison to the initial calibration, or to a third party check standard. Replace the secondary calibration standard, and (or) the primary calibration standard if signs of degradation are present. If the third party check standard indicates a pattern of decreasing recoveries for methyl acetate, monitor the area of methyl acetate relative to internal standard compound, fluorobenzene, for primary calibration standard mix 5 (table 3). Replace the primary calibration standard if the ratio of methyl acetate to fluorobenzene is increasing with the age 
of the standard. Monitor the recovery of acrolein in the secondary calibration standard and the third party check standard. Prepare secondary calibration standards every 2 months, or sooner, if any compounds show more than a 20 percent shift in response.

\subsection{Calibration Standard Solutions, Method 0-4436}

Obtain commercially prepared primary calibration standards in methanol for the compounds listed in table 4, at $2,000 \mu \mathrm{g} / \mathrm{mL}$. Alternatively, prepare from individual neat standards. The composition and number of separate primary calibration standards are determined by shelf-life limitations, compound class, or commercially available mixes. Refer to table 4 for the concentrations, solvents, groupings, and calibration ranges used in this method.

Prepare two secondary calibration standard solutions, in purge-and-trap grade methanol, combining the primary calibration standards, to make one solution containing all compounds for each secondary calibration standard solution. Prepare the secondary calibration standard solutions so that they are kept concentrated enough so that only a small quantity, less than $25 \mu \mathrm{L}$, of each solution is required to obtain even the most concentrated calibration standard in VBW.

Monitor the secondary calibration standard frequently by comparison to the initial calibration, or to a third party check standard. Replace the secondary calibration standard, and (or) the primary calibration standard if signs of degradation are present. The early eluting permanent gases are generally the first compounds to show signs of losses. Prepare secondary calibration standards every 3 months, or sooner, if any compounds show more than a 20 percent shift in response.

\subsection{Third Party Check Standard Solution}

Prepare a third party check (TPC) standard for each method. Obtain commercially prepared or custom primary third party check standards in methanol, methanol/water, or water. Alternatively, prepare from individual standards. The TPC standard should be prepared from a different lot than the primary calibration standards, and ideally from a different vendor than the primary calibration standards. The TPC standard serves as a check of the calibration curve validity. Prepare a secondary TPC standard solution, in purge-and-trap grade methanol, combining the primary TPC standards, to make one solution containing all compounds. Prepare the secondary TPC standard solution so that $25 \mu \mathrm{L}$ into $50 \mathrm{~mL}$ of water will produce a concentration in the low to mid-range of the calibration curve, and such that $2-5 \mu \mathrm{L}$ spiked into $50 \mathrm{~mL}$ will produce a limit of quantitation standard (LOQ) 1-2 times the reporting level for each compound. Prepare fresh secondary TPC standard solutions every 2 months for Method O-4437 and every 3 months for Method O-4436, or sooner, if any compounds show signs of degradation.

\subsection{Laboratory Reagent-Water Spike Standard Solution}

Prepare a separate solution for each method. The TPC standard solution can be used for the laboratory reagent-water spike (SPK) standard solution, or the secondary calibration standard solution may be used. Prepare the SPK secondary standard solution so that $10-25 \mu \mathrm{L}$ into $50 \mathrm{~mL}$ of water will produce a concentration in the low to mid-range of the calibration curve. Prepare fresh secondary SPK standard solution every 2 months for Method O-4437, and every 3 months for Method O-4436, or sooner if any compounds show signs of degradation.

\section{Sample Collection, Preservation, Labeling, Shipment, and Holding Times}

Sampling for VOCs requires special handling because samples can be easily contaminated if the protocol is not followed. Detailed descriptions of sampling methods for collecting VOCs in surface water and groundwater are described in the National Field Manual by Wilde and others (1999).

\subsection{Collection of Environmental Samples}

Collect samples for VOC analysis in triplicate for each method in clean $40-\mathrm{mL}$ borosilicate amber vials (VOC vials) with Teflon-faced septa. If the sample contains free chlorine, collect the sample in VOC vials containing ascorbic acid, a dechlorination reagent. Triplicate vials are required because each sample may be subjected to multiple analyses (dilutions and reanalysis because of QC failures, carryover problems), each of which consumes an entire vial. Fill the vials to overflowing, leaving a convex meniscus. Add 2-5 drops of $\mathrm{HCl}$ (1:1) to the filled vial drop by drop to adjust the $\mathrm{pH}$ to $\leq 2$. Cap immediately. If the sample is expected to contain free chlorine, the vials used should contain a premeasured amount of ascorbic acid. Be careful not to flush out the ascorbic acid when filling the vial. Do not allow air to pass through the sample or to become trapped inside the vial. Number the VOC vials in the order filled.

\subsection{Collection of Field Quality Control Samples}

8.2.1. Source Solution Blanks - Three VOC vials are collected for each method and preserved with $\mathrm{HCl}$ (1:1). A source solution blank is prepared from the same VPBW used for the field equipment blank. The VPBW is poured directly into three VOC vials; it is not passed through any field equipment. 
8.2.2. Field Equipment Blanks - Three VOC vials are collected for each method and preserved with $\mathrm{HCl}(1: 1)$. The field equipment blank goes through the same procedures as the environmental samples, described in the USGS National Field Manual chapters A3 (Wilde, 2004) and A4 (U.S. Geological Survey, 2006). VPBW is used for equipment blanks. The sampling equipment is not rinsed with any solvents except for methanol (high pressure liquid chromatography grade or pesticide grade). Other volatile solvents, such as hexane, and isopropyl alcohol are method compounds so they should not be used on sampling equipment. The field equipment blanks are used for determining if the equipment used to collect the samples resulted in contaminating the environmental samples. Failure to rinse the entire amount of methanol out of the equipment can result in detections of several nontarget VOCs, including acetone and 2-butanone.

8.2.3. Trip Blanks_-Trip blanks accompany samples throughout the sampling and shipping period. Trip blanks are used to determine if contamination is caused by shipping and storage of unopened vials. Trip blanks remain sealed throughout transportation and storage. Trip blanks are not preserved. Submit two or three vials per analysis.

8.2.4. Environmental Sample Replicates-Three VOC vials are collected for each method and preserved in the same manner as the environmental samples.

8.2.5. Environmental Samples for Laboratory Matrix Spikes-Three VOC vials are submitted to the laboratory for each method. Upon receipt, the sample is spiked with method compounds and analyzed within 14 days of the sample collection date.

\subsection{Sample Preservation}

Preserve samples in the field by adding 2-5 drops of $\mathrm{HCl}(1: 1)$ until a $\mathrm{pH}$ of 2 is achieved. Chill immediately to $4{ }^{\circ} \mathrm{C} \pm 2{ }^{\circ} \mathrm{C}$. Use only quality-controlled $\mathrm{HCl}$ (1:1) for sample preservation, stored in Teflon ${ }^{\circledR}$ dropper bottles. Laboratory studies have shown that $\mathrm{HCl}$ quality degrades with age and when stored in inappropriate containers. The expiration date for the $\mathrm{HCl}(1: 1)$ is 4 months from the shipping date. $\mathrm{HCl}$ that is improperly stored may result in detections of chloromethane, chloroethane, and hexafluoropropene in preserved samples at concentrations large enough to be detected by this method. Store $\mathrm{HCl}$ in a cool, dark place away from VOC sources. Dispense the acid from the Teflon ${ }^{\circledR}$ dropper bottle to the sample in the VOC vial. Many water samples require several drops of the $\mathrm{HCl}(1: 1)$ to achieve a $\mathrm{pH}$ of 2 . To test how much $\mathrm{HCl}(1: 1)$ is needed, collect an extra water sample in a spare 40-mL VOC vial, and add the preservative one drop at a time until a $\mathrm{pH}$ of 2 is achieved. Discard this extra sample in an appropriate container and collect and preserve the replicate VOC samples using the determined number of drops of $\mathrm{HCl}(1: 1)$. The majority of samples require less than five drops of the $\mathrm{HCl}(1: 1)$. Samples with high alkalinity may require more than five drops. Do not add more $\mathrm{HCl}(1: 1)$ than necessary since over-acidification could detrimentally affect the sample analysis.

\subsection{Labeling}

Indicate the site name, sample date and time, and schedule on each VOC vial. Number the vials in the order filled. Affix the label to the glass portion only, not near the cap. Use a ball-point pen to handwrite the label. Do not use permanent markers because the ink could contaminate the sample with method compounds. Alternatively, preprint the labels on a printer. Let the ink dry before placing the label on the vial. Do not wrap the label around the vial more than once, or wrap extra tape around the outside of the vial because it will interfere with the autosampler. Do not wrap tape around the cap of the vial because solvents in the adhesive in the tape can outgas and contaminate the sample with toluene and other VOCs.

\subsection{Shipping}

Store the samples at $4{ }^{\circ} \mathrm{C} \pm 2{ }^{\circ} \mathrm{C}$ until the samples are ready to ship. Pack enough ice in each shipping container to ensure that the samples remain chilled but not frozen throughout transit, which could result in broken vials. Do not use dry ice for shipping volatiles because samples packed on dry ice might freeze. Wrap the VOC vials in bubble wrap to prevent breakage in transit. Do not use foam-packing peanuts for shipping VOC samples because the samples may become contaminated with method compounds. Ship samples using an overnight delivery service to ensure the samples are received chilled, and to allow sufficient time at the laboratory for processing and analysis.

\subsection{Holding Times}

Store samples for VOC analysis at $4{ }^{\circ} \mathrm{C} \pm 2{ }^{\circ} \mathrm{C}$ upon receipt. Analyze the samples within 14 days of sample collection, in order of arrival, unless special arrangements are made.

\section{Sample Analysis}

The general procedures described here apply to both Method O-4437 and Method O-4436. 


\subsection{Method Configuration}

9.1.1. Instrument Operating Conditions - Suggested operating conditions are listed for the purge-and-trap sample concentrator, gas chromatograph, mass spectrometer in tables 5 and 6 for Method O-4437, and in tables 7 and 8 for Method O-4436. Instrument settings may need to be adjusted to achieve similar results. Each compound was determined individually with the mass spectrometer in the full scan mode to establish retention times and verify the identity of the compound. For Method O-4437 the SIM windows were established using the ions listed in table 6. Other SIM ions may be selected if interference is present. Any changes to the operating conditions must be documented in the method standard operating procedure and comparable quality obtained. The data reduction method was created using the internal standard compound assignments, and quantitation and qualification ions listed in table 6 (Method O-4437) and table 8 (Method O-4436).

9.1.2. Internal Standard and Surrogate Standard Compound Assignments, Method O-4437-Four internal standard compounds and five surrogate standard compounds are selected for Method O-4437. 1-Bromo-3-chloropropane- $d_{6}$ is assigned as the internal standard compound for the fumigant compounds, which are all halogenated alkanes. The corresponding surrogate standard compound is 1,2-DCA- $d_{4}$. 1,4-Dioxane- $d_{8}$ is assigned as the internal standard compound for 1,4-dioxane, N-nitrosodiethylamine, and 2-ethoxyethyl acetate. All three compounds have a similar purge response. Tetrahydrofuran- $d_{8}$ is the corresponding surrogate standard compound. Tetrahydrofuran- $d_{8}$ was added to the method in January 2013, after the initial method validation (bias and variability, MDLs, and holding time study) had been completed, but before the preliminary implementation of the method began. Additional data have been collected from laboratory QC samples and matrix spikes (MSPKs) during implementation to assess the performance of tetrahydrofuran- $d_{8}$ in VBW and multiple groundwater and surface water samples. tert-Butyl alcohol- $d_{10}$ is assigned as the internal standard compound for all alcohols, ketones, and aldehydes, with isobutyl alcohol- $d_{6}$ as the corresponding surrogate standard compound. The remaining compounds are assigned to internal standard compound fluorobenzene. Toluene- $d_{8}$ and BFB are the corresponding surrogate standard compounds. $\mathrm{BFB}$ is included to evaluate the mass spectrometer performance.

9.1.3. Internal Standard and Surrogate Standard Compound Assignments, Method O-4436-All four surrogate and internal standard compounds from Method O-4217-96 (NWQL LS 2020) are retained for Method O-4436. The function of 1,2-DCA- $d_{4}$ was changed from a surrogate standard compound in Method O-4127 to an internal standard compound in Method O-4436. All halogenated alkanes and vinyl chloride are assigned to 1,2-DCA- $d_{4}$. 1-Bromo-3-chloropropane- $d_{6}$ is selected as the new surrogate standard assigned to $1,2-\mathrm{DCA}-d_{4}$. The halogenated alkanes exhibit behavior more similar to 1,2DCA- $d_{4}$ than to fluorobenzene, which makes it a better choice as an internal standard compound. All remaining compounds are assigned to internal standard compound fluorobenzene. Toluene- $d_{8}$ and BFB are the corresponding surrogate standard compounds. BFB is included to evaluate the mass spectrometer performance.

9.1.4. Compound Retention Times-Establish compound retentions times by analyzing a calibration standard. Retention times are provided in table 6 for Method O-4437 and table 8 for Method O-4436. Use single component standards to verify retention times for isomers and (or) closely eluting and co-eluting compounds. It is critical to verify compound retention times on each analytical system as there are variances among operating conditions, columns, and instrumentation.

An example of a typical selected ion chromatogram is shown in figure 1 for a calibration standard for Method O-4437. An example of a total ion chromatogram is shown in figure 2 for a calibration standard for Method O-4436. A compound number listed in tables 6 and 8 corresponds to the number shown above a peak in figures 1 and 2. Surrogate and internal standard compounds are also included in both figures.

9.1.5. Mass Spectrometry Tune Check - Generate a tune report for the MS prior to an instrument batch run. Determine if the air background is acceptable by checking the ratios of nitrogen, oxygen, and water. Determine if the MS needs to be retuned by checking the ratios of masses of perfluoro-tert-butylamine. Retune the MS if necessary using the automated tuning software provided by the instrument vendor or an alternative tuning method. After retuning, inject a solution of BFB. The abundances of the relative mass fragments should be within ranges specified by EPA in Method 524.3 (Prakash and others, 2009), listed in table 9. BFB criteria are also verified after instrument maintenance and prior to calibration.

9.1.6. Initial Instrument Check - Prior to starting an instrument batch run, bake the trap for at least 10-15 minutes at the temperature recommended by the manufacturer. Analyze a test blank to determine if the system is free of gross contamination. Analyze a continuing calibration verification (CCV) standard. If the CCV standard is within QC specifications, then a new initial calibration curve is not needed. If the CCV standard fails QC specifications, prepare a new calibration curve. 


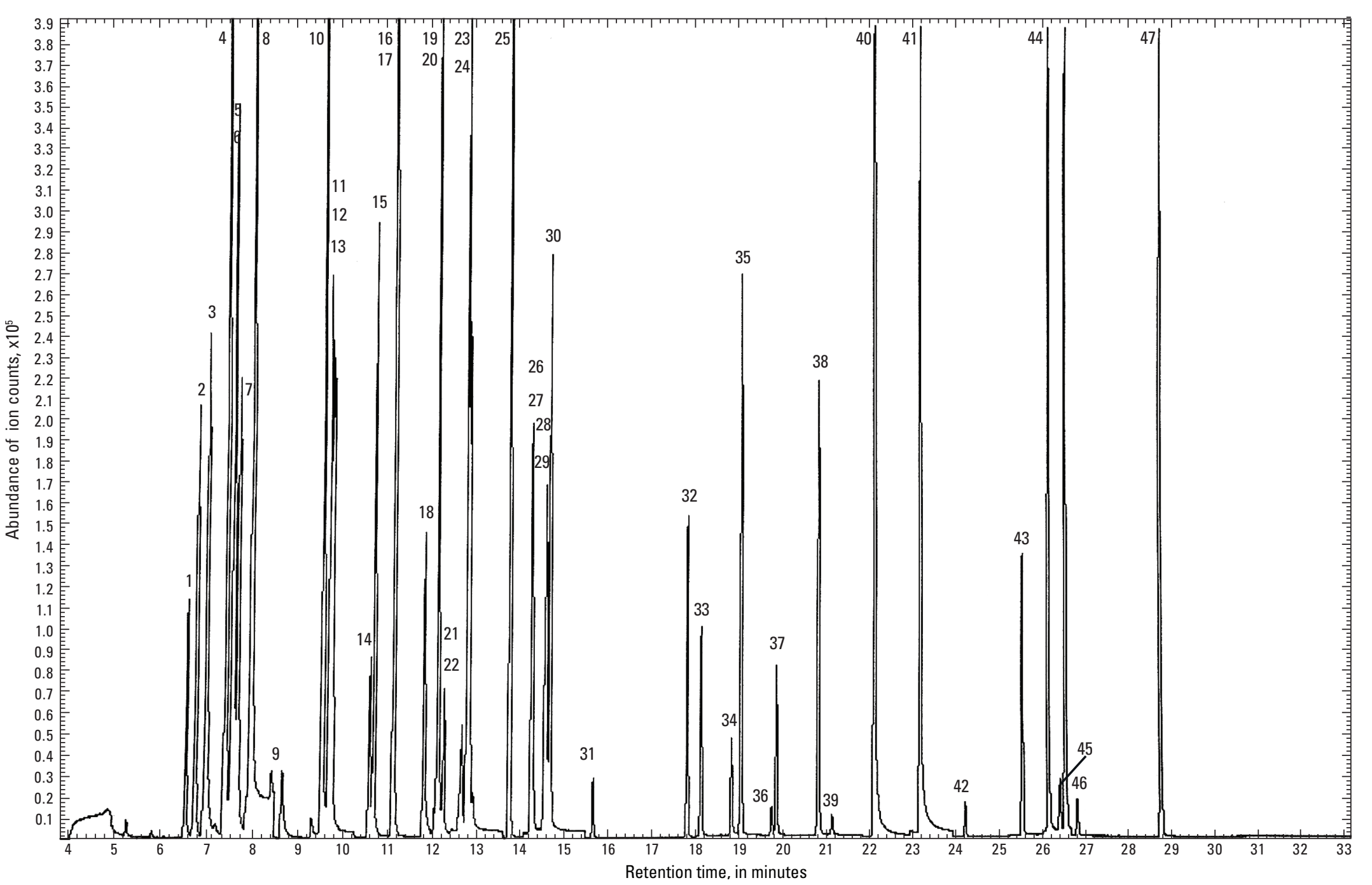

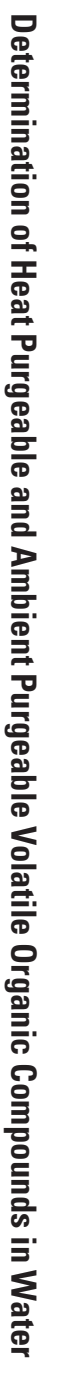

Figure 1. Selected ion chromatogram of a calibration standard for heat purgeable volatile organic compounds. Numbers shown above each peak correspond to compounds listed in table 6 . The unnumbered peak was not retained in the method. Peaks 13, 14, 15, 25, and 35 are surrogate standard compounds. Peaks 5, 17, 21, and 32 are internal standard compounds. 


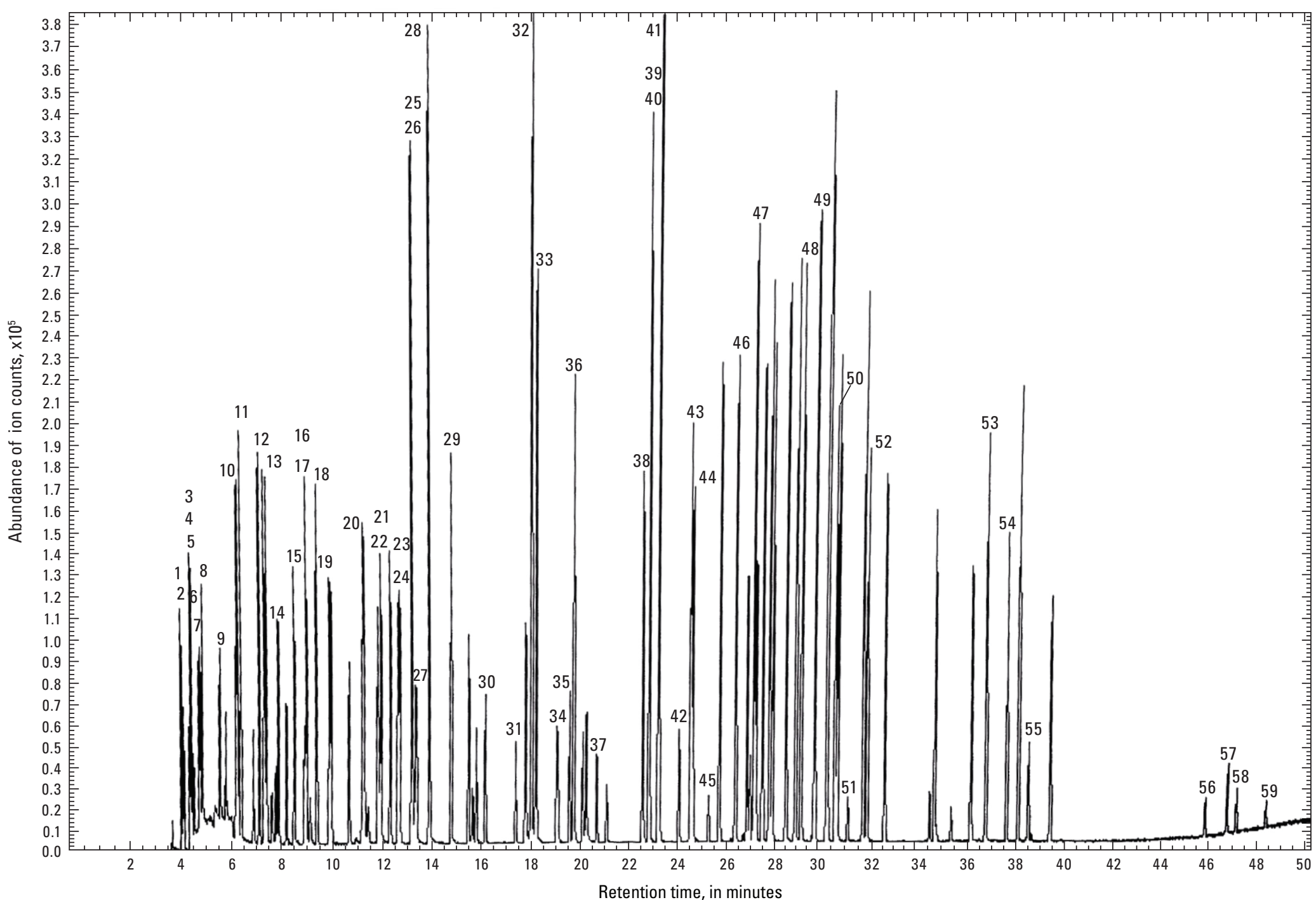

Figure 2. Total ion chromatogram of a calibration standard for ambient purgeable volatile organic compounds. Numbers shown above each peak correspond to compounds listed in table 8. Peaks not numbered are compounds on other National Water Quality Laboratory schedules. Peaks 32, 42 , and 46 are surrogate standard compounds. Peaks 26 and 28 are internal standard compounds. 


\subsection{Sample Preparation}

Choose samples in a chronological sequence, with the oldest samples at the beginning of the instrument batch run. Prepare a dilution based on previous history or current information from the site if a sample is suspected of being highly contaminated to prevent gross contamination of the purge-andtrap system. Initially dilute samples containing oil field brines, landfill leachates, or high conductivities because these samples are often foamy, which can cause interference during analysis. Place known, highly contaminated samples toward the end of the run with additional carryover blanks to prevent carryover contamination into the next sample. The suggested instrument batch run sequence is listed in table 10 . For any sample, use a note on the sample preparation sheet to comment on the presence of bubbles, sediment, or anything else unusual about the sample. When the instrument batch run has completed, use the remaining sample in the vial to check preservation, using a $\mathrm{pH}$ strip. Record the results of the preservation check on the sample preparation sheet. Submit an improper preservation report for samples that were not preserved properly.

\subsection{Preparation of Standards and Blanks}

Prepare the following standards and blanks in VBW on the day of analysis. Minimally, prepare the quantities listed in the instrument batch run sequence (table 10). All standards are prepared by spiking an aliquot of the secondary calibration standard or the secondary third party check standard into $50 \mathrm{~mL}$ of VBW in a glass syringe, and immediately transferring into a 40-mL VOC vial, filling without headspace. The VOC vial contains one drop of the $\mathrm{HCl}(1: 1)$ to attain a $\mathrm{pH}$ of 2 in the VBW. All standards and blanks are preserved, except for the carryover blank. The standards and blanks are transferred to a chilled autosampler or stored at $4{ }^{\circ} \mathrm{C} \pm 2{ }^{\circ} \mathrm{C}$ in a refrigerator until the instrument is ready, but no longer than $24 \mathrm{hr}$.

9.3.1. Calibration Standards-Prepare fresh calibration standards prior to starting an instrument run by spiking $1-20 \mu \mathrm{L}$ of the appropriate secondary calibration standard solution into $50 \mathrm{~mL}$ of VBW. Refer to table 3 for Method O-4437 and table 4 for Method O-4436 for the calibration range for each compound in water. Prepare new calibration curves after instrument maintenance or when the continuing calibration verification standards indicate the instrument needs to be recalibrated.

9.3.2. Continuing Calibration Verification (CCV) StandardSpike 5-20 $\mu \mathrm{L}$ of the secondary calibration standard into $50 \mathrm{~mL}$ of VBW. CCV standards are analyzed before and after every block of 10 environmental samples.

9.3.3. Third Party Check (TPC) Standard-The TPC standard solution is prepared from a different source than the calibration standards. Prepare a fresh TPC standard by spiking $25 \mu \mathrm{L}$ of the secondary TPC standard into $50 \mathrm{~mL}$ of VBW. The TPC standard validates the accuracy of the calibration curve. Prepare one TPC standard when new calibration standards are analyzed.

9.3.4. Laboratory Reagent-Water Spike (SPK) StandardThe SPK standard measures bias and variability over time. The SPK standard solution is prepared by spiking $10-25 \mu \mathrm{L}$ of the secondary TPC standard or the calibration standard into $50 \mathrm{~mL}$ of VBW. Prepare one SPK with every instrument batch run. If the TPC standard is used to prepare the SPK standard, the calibration curve is verified in each instrument run.

9.3.5. Limit of Quantitation (LOQ) Standard-Prepare an LOQ standard by spiking $1-5 \mu \mathrm{L}$ of the secondary TPC standard solution or the secondary calibration standard into $50 \mathrm{~mL}$ of VBW. An LOQ standard is analyzed at the beginning and at the end of an instrument run to verify that the reporting level (RL) can be detected, and validates the performance at the low end of the calibration curve. The LOQ standard is ideally prepared at concentrations that are within two to five times the MDL.

9.3.6. Laboratory Matrix Spike (MSPK) — One or two additional environmental samples (additional triplicate VOC vials for each spike) are provided to be spiked at the laboratory as a QC check of matrix interferences. Upon sample receipt, remove the sample cap and quickly inject $20 \mu \mathrm{L}$ of the secondary calibration standard into the middle of the vial and recap. Gently invert the vial three to five times to mix, storing in the refrigerator until analysis. Alternatively, the sample may be spiked with $20 \mu \mathrm{L}$ of the TPC standard.

9.3.7. Laboratory Reagent-Water Blank (LRB)-Prepare by filling a $40-\mathrm{mL}$ VOC vial containing one drop of $\mathrm{HCl}$ (1:1) with VBW without headspace. LRBs are analyzed after every group of 10 samples to assess laboratory background concentrations of VOCs.

9.3.8. Carryover Blank (COB)_-Prepared by filling a 40-mL VOC vial with VBW, without headspace. The carryover blank is not preserved since its main function is to remove residual VOCs in the sample path. COBs are analyzed after CCV standards, higher concentration calibration standards, or highly contaminated samples. COBs are not used as QC samples.

\subsection{Calibration}

9.4.1. Prepare an Initial Calibration Curve-Depending on the specific compound chemical characteristics and method, prepare 5 to 10 calibration standards, defining the expected concentration ranges for each quantitated 
compound, in VBW to create individual compound concentrations ranging from 0.005 to $200 \mu \mathrm{g} / \mathrm{L}$. The suggested calibration range for each compound is listed in table 3 for Method O-4437 and table 4 for Method O-4436.

9.4.2. Calculate the Response Factor $(R F)$-Use equation 1 for each compound and surrogate standard compound.

where

$$
R F=\frac{C_{I S} \times A_{i}}{C_{i} \times A_{I S}}
$$

$$
\begin{gathered}
C_{I S}=\begin{array}{c}
\text { concentration of the internal standard } \\
\text { solution, in } \mu \mathrm{g} / \mathrm{L} ;
\end{array} \\
A_{i}=\text { GC peak area of the quantitation ion for } \\
\text { the selected compound or surrogate } \\
\text { standard compound; } \\
C_{i}=\text { concentration of the selected compound or } \\
\text { surrogate standard compound, in } \mu \mathrm{g} / \mathrm{L} ; \\
\text { and } \\
A_{I S}=\text { GC peak area of the quantitation ion for the } \\
\text { internal standard compound. }
\end{gathered}
$$

The quantitation ions used in these calculations are listed in table 6 for Method O-4437 and table 8 for Method O-4436.

\subsubsection{Evaluate the Calibration Curve for Each Compound:}

- Use of the mean RF is acceptable if the relative standard deviation (RSD) for each compound is less than or equal to 20 percent.

- Curve-fitting routines provided by the instrument manufacturer must have a minimum curve-fitting value $\left(R^{2}\right)$ of 0.995 .

- The initial calibration standards are checked for accuracy by requantitating the calibration standards against the new calibration curve.

- Observed concentrations should be within \pm 20 percent of the expected concentrations.

- Points may be deleted from the low end or high end of the calibration curve if there are detections in the LRB, saturation of the detector, water vapor interferences, or failure to meet identification criteria, as long as a minimum of five calibration standards are retained.

- Removal of an individual point from the interior of the calibration curve is not permitted.

- An entire calibration level may be removed from the interior of the calibration curve if there is a documented valid reason (bad injection, all compounds show a bias in the same direction).
- If an entire calibration level is removed from the high end or low end of the calibration curve, the calibration range is adjusted accordingly.

- Adjustments to the reporting levels may be necessary and reported data may be qualified.

- A calibration level may be replaced if there is a documented valid reason. The replacement level is analyzed within $24 \mathrm{hr}$ of the initial level and before sample analysis. Only one level may be replaced from the low end or high end of the calibration curve. Only one level may be replaced in the interior of the calibration curve.

- Replacement of individual compounds in the interior of the calibration curve is not permitted.

\subsection{Identification and Quantitation}

Qualitative Identification Criteria-Initially identify a selected compound by comparing the GC retention time (RT) of the compound to the RT of the standard solution. The RT of the sample needs to be within \pm 0.1 minute of the expected RT for the compound in question. Verify the mass spectrum for each selected compound by comparing the mass spectrum with a reference spectrum obtained from standards analyzed on the GC/MS system. For a compound to be considered detected, all qualification ions and the quantitation ion should be present within \pm 25 percent of the ratios of the in-house reference standards. The total ion chromatogram and the extracted ion peaks should be Gaussian in shape. The extracted ion peaks should be at least two to three times the instrument noise. It is often beneficial to compare the extracted ion profiles of important ions (or suspected interfering ions) to determine whether they maximize at the expected retention time with intensities consistent with the reference mass spectrum.

Quantitation - Calculate the concentration in the sample using equation 2 and the mean RF (eq. 1). The terms in equation 2 and 3 are defined in the section 9.4.2.

$$
C_{i}=\frac{C_{I S} \times A_{i}}{R F \times A_{I S}}
$$

Percent recovery of the surrogate standard compound is calculated as follows:

$$
\text { Percent Recovery }=\frac{C_{I S} \times A_{i}}{R F \times A_{I S} \times C_{i}} \times 100
$$

where

Percent Recovery $=$ percent recovery of the surrogate standard compound. 


\section{Quality Assurance and Quality Control Samples and Criteria}

Each instrument run batch consists of the following quality assurance and quality control (QC) checks.

10.1. Continuing Calibration Verification (CCV) StandardAnalyze a CCV standard at the beginning of an instrument batch. To confirm the calibration curve is consistent throughout the entire instrument batch, analyze additional CCV standards after each block of 10 samples or less. Analyze midlevel CCV standards after every 10 samples and place a higher-concentration CCV standard at the end of the batch. Alternatively, the CCV standard concentration might be varied during the analysis to collect QC information at different concentrations. Determine statistical control limits after $30 \mathrm{CCV}$ standards have been collected or use static limits of 70-130.

10.2. Third Party Check (TCP) Standard - The TPC standard is prepared from a separate source than the calibration standards. The TPC is used to access the validity of the calibration curve. Determine statistical control limits when 30 or more TCP standards have been analyzed. Alternatively, static limits of 70-130 percent can be used until enough data are collected.

10.3. Laboratory Reagent-Water Spike Standard (SPK) - The SPK standard is prepared from either the TPC standard or the calibration standard. The SPK standard is used to evaluate overall performance. Determine statistical control limits when 30 or more SPKs have been analyzed. Alternatively, static limits of $70-130$ percent can be used until enough data are collected.

10.4. Laboratory Reagent-Water Blanks (LRB)_LRBs are analyzed before and after every block of 10 samples. An LRB contains the same preservatives as the samples. Detections in an LRB can occur because of laboratory background, contaminants in the preservative, and (or) carryover contamination from samples with high concentrations. Concentrations of VOCs in an LRB should be less than the reporting level.

10.5. Limit of Quantitation Standard (LOQ)—An LOQ standard is used to determine if the instrument sensitivity is sufficient to meet all identification criteria at the reporting level. The LOQ standard is analyzed at the beginning of the run, before the CCV standard, and again after the ending LRB. There are no initial acceptance criteria for an LOQ standard. Statistical control limits may be determined when 30 or more LOQ standards have been analyzed. The recovery for this standard indicates the bias at the low end of the curve. An LOQ standard is prepared from the TPC standard solution, a diluted calibration standard, or SPK standard solution.

10.6. Surrogate Recoveries - The surrogate standard compounds are added to every QC sample and environmental sample by the autosampler before purging begins. The surrogate standard compounds are used to assess the overall performance of each sample. Determine statistical control limits when a total of at least 30 LRBs and 30 SPKs have been analyzed. An instrument run generally has two or more LRBs and one SPK. Alternatively, $70-130$ percent can be used as static control limits until enough data are collected to calculate statistical control limits.

10.7. Internal Standard Compound Areas - The internal standard compounds are added to every QC sample and environmental sample by the autosampler before purging begins. The area of the quantitation ion of the internal standard compound in the first CCV standard is compared to the internal standard compound areas of all remaining QC samples and environmental samples in the instrument run batch. The internal standard compound area should be within \pm 50 percent of its area in the first CCV standard (Munch, 1995).

\section{Reporting Results}

Compound concentrations in each sample are determined using the calibration curves after the chromatogram has been reviewed to confirm the qualitative identification of the compound. All concentrations that meet identification criteria are reported in $\mu \mathrm{g} / \mathrm{L}$ for Method O-4437 and Method O-4436.

Samples with concentrations that exceed the highest calibration standard are diluted and reanalyzed. Samples with concentrations below the MDL are reported with a National Water Information System (NWIS) value-qualifier code of "b" (value was extrapolated at the low end). Results for compounds not detected in the samples are reported as less than the RL.

Results for some compounds are reported with a NWIS water-quality-database qualifier remark code of " $E$ " to indicate estimated concentrations. Hexachlorocylcopentadiene in Method O-4437 is reported with an "E" remark code due to high variability. Bromomethane, carbon disulfide, and chloromethane in Method O-4436 are reported with an "E" remark code because of potentially high variability due to sulfur. Hexane is reported with an "E" remark code because of losses seen in the holding time study.

2-Ethyl-1-hexanol, chloromethane, methylene chloride, and toluene are blank-limited compounds. These four compounds are reported to NWIS using the minimum reporting 
level (MRL) convention (Childress and others, 1999). The MRL is the smallest measured concentration of a compound that may be reliably reported by using a given analytical method. No data are reported at concentrations less than the MRL

\section{Results and Discussion of Method Development and Validation Data}

The initial method development approach included experiments that determined which compounds would be suitable for purge-and-trap techniques. After the NTAS team ranked the prioritized compounds and subdivided them further into candidates for the heat purgeable and ambient purgeable methods, individual reference standards were obtained for each compound. Each compound was tested initially by analyzing a direct injection into the gas chromatograph for each analytical method to determine if the compound were suited for gas chromatographic analysis. A standard of each compound was prepared in VBW, ranging in concentration from 20 to $100 \mu \mathrm{g} / \mathrm{L}$, and purged using the current conditions for the heat purgeable and ambient purgeable methods. Several compounds detectable by direct injection were undetectable using either purgeable method. For the compounds that were detectable after purging, a response factor was determined, relative to internal standard compound fluorobenzene. Response factors were reviewed to determine which method would be optimal for each compound. Compounds with response factors less than 0.001 on the ambient purgeable method were assigned to the heat purgeable method.

A graph of water solubility compared to Henry's Law Constants is shown in figure 3 for all compounds tested. The graph illustrates the experimental results showing that compounds with Henry's Law Constants of $10^{-6}$ atmosphere-meter cubed $/ \mathrm{mole}\left(\mathrm{atm}-\mathrm{m}^{3} / \mathrm{mole}\right)$ or lower were not detected using purge-and-trap techniques. These compounds included cumene hydroperoxide, urethane, and 2-ethoxyethanol. Many of the higher molecular weight compounds containing nitrogen were not detected or performed poorly, including N-nitrosodimethyl amine, benzamine, o-toludine, quinoline, pyridine, and nitrapyrin. Lower molecular weight, nitrogen-containing compounds performed well, including acetonitrile, chloropicrin, nitrobenzene, 2-nitropropane, and $\mathrm{N}$-nitrosodiethylamine.

A few compounds tested were found to be unstable. The half-life for chloromethyl methyl ether in water at $20^{\circ} \mathrm{C}$ is estimated to be less than 1 second because of rapid hydrolysis (Tou and Kallos, 1974). Cyanogen bromide was detected in a single component standard using the heated purge method; however, it reacted with other compounds when added to a multicomponent standard.

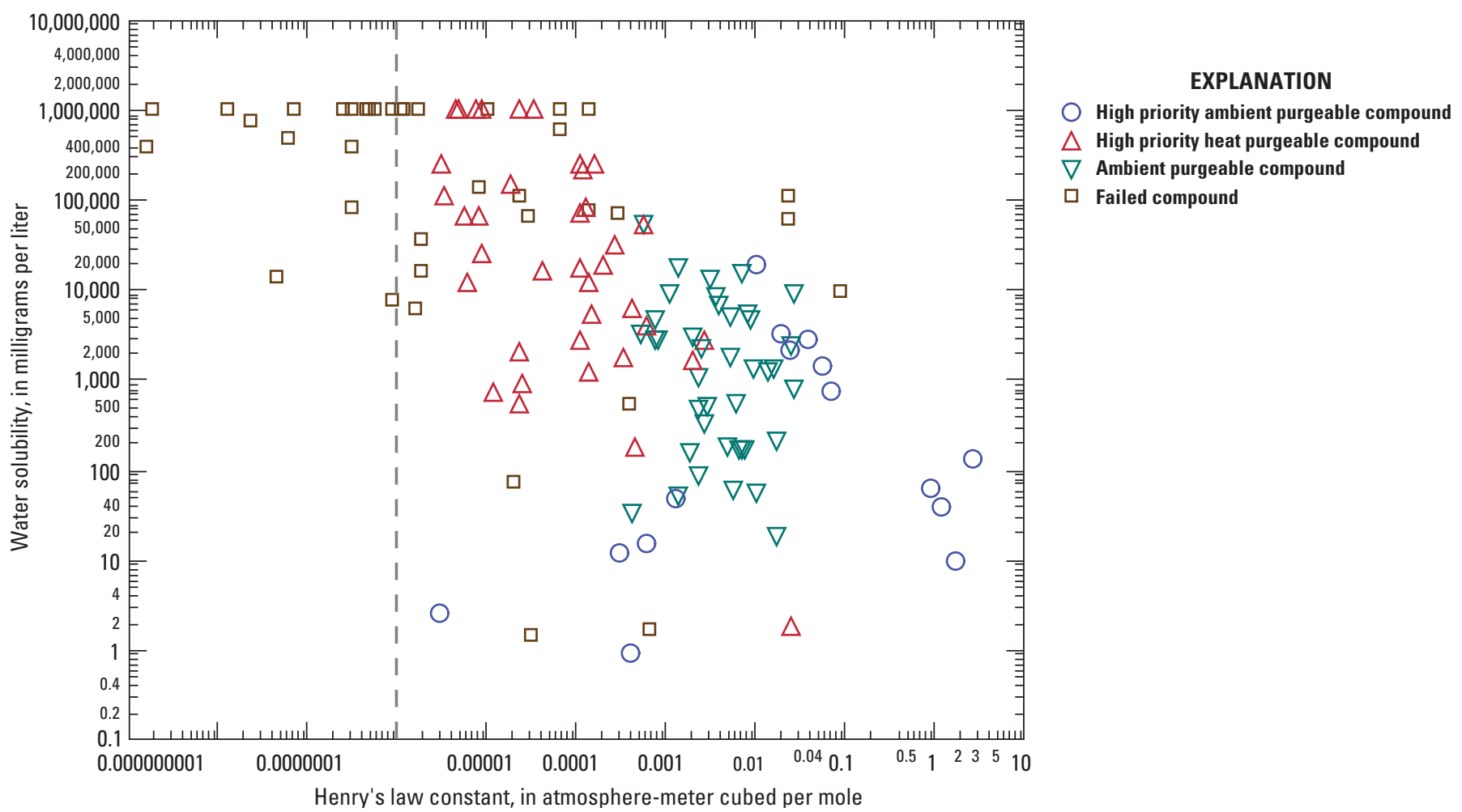

Figure 3. Water solubility compared to Henry's Law Constant for priority compounds selected for testing using purge-and-trap technology. The line at $10^{-6}$ atmosphere-meter cubed per mole shows compounds that could not be purged from samples under conditions tested. 
The fumigant compounds, including 1,2-dibromo3-chloropropane (DBCP), 1,2-dibromoethane (EDB), 1,2-dichloropropane (1,2-DCP), and 1,2,3-trichloropropane (1,2,3-TCP), determined by Method O-4127 (NWQL LS 2020), were added to Method O-4437 to achieve lower MDLs.

Refer to the Appendix table 1-2 for physical properties for all compounds tested with these methods.

Method validation experiments for those compounds consisted of determining MDLs and RLs, bias and variability at different concentration levels and in different matrices, and stability of samples from sample collection to analysis. Laboratory QC samples and field-submitted QC samples were evaluated to determine long-term performance and interferences. The data quality objectives for the method validation studies were that the bias should not be more than \pm 30 percent ( $70-130$ percent), and the RSD should not exceed 30 percent. Compounds not meeting more than one of the objectives in more than one matrix or holding time study were removed from the method. Compounds not meeting one objective were reported as an estimated concentration with an NWIS water-quality-database remark code of "E."

\subsection{Method Detection Limits and Reporting Levels}

MDLs were determined for the compounds on Methods O-4437 and O-4436, according to the EPA procedure (U.S. Environmental Protection Agency, 2002). A minimum of six replicates was spiked in VBW at concentrations ranging from one to five times the estimated MDLs and analyzed over a minimum of three separate instrument runs. MDLs are calculated using equation 4.

$$
\mathrm{MDL}=s \times t(n-1,1-\alpha=0.99)
$$

where

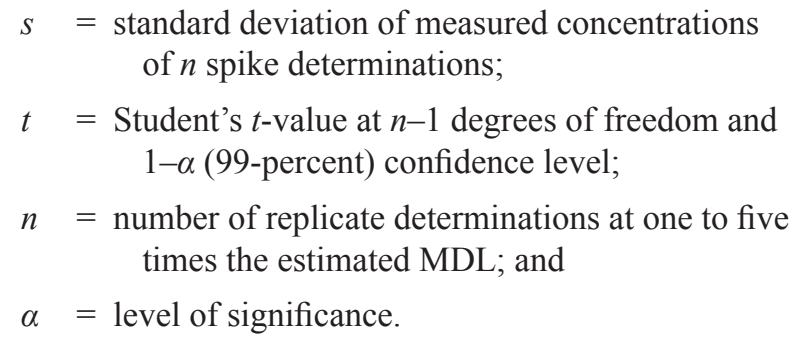

The Student's $t$-value defines a 1 percent chance of false positives. The MDL then is defined as the minimum concentration of a compound that can be identified and reported with 99-percent confidence that the compound concentration is greater than zero (U.S. Environmental Protection Agency, 2002). The RL is defined as two times the MDL (Childress and others, 1999).

12.1.1. Method Detection Limits and Reporting Levels for Method O-4437_-The heat purgeable method encompasses a variety of chemical structures, including alcohols, aldehydes, alkyl and cyclic ethers, fumigant compounds, ketones, and nitrogen containing compounds. Henry's Law Constants range from $10^{-6}$ to $10^{-2} \mathrm{~atm}-\mathrm{m}^{3} / \mathrm{mole}$. The calculated MDLs range from 0.002 to $3 \mu \mathrm{g} / \mathrm{L}$. Four heat purgeable VOCs have MDLs greater than $1 \mu \mathrm{g} / \mathrm{L}$, including 2-ethoxyethyl acetate, alpha-terpineol, isophorone, and N-nitrosodiethylamine. The MDLs for the fumigant compounds EDB, 1,2-DCP, 1,2,3-TCP, chloropicrin, and DBCP range $0.002-0.010 \mu \mathrm{g} / \mathrm{L}$. Of the remaining VOCs, 12 have MDLs $\leq 0.1 \mu \mathrm{g} / \mathrm{L}$ and 17 have MDLs between 0.11 and $1 \mu \mathrm{g} / \mathrm{L}$. RLs were established at two times the MDL, with the exception of 2-ethyl-1-hexanol.

12.1.2. Blank-Limited Compounds and Minimum Reporting Levels, Method O-4437-2-Ethyl-1-hexanol was detected with 100 percent frequency in LRBs, and with 99 percent frequency in FSBs (tables 20, and 21). Data for 2-ethyl-1-hexanol are reported to NWIS database using the MRL convention (Childress, and others, 1999). An MRL of $<2 \mu \mathrm{g} / \mathrm{L}$ was established based on statistical analysis of 84 LRBs (table 20) from January 2013 through August 2013. The 99th percentile concentration for 2-ethyl-1-hexanol was $2.1 \mu \mathrm{g} / \mathrm{L}$. Sample concentrations are censored below the MRL.

12.1.3. Discussion of Selected Heat Purgeable Volatile Organice Compounds-1,2,3-TCP is on the EPA's UCMR 3 list with a MRL of $0.03 \mu \mathrm{g} / \mathrm{L}$ (U.S. Environmental Protection Agency, 2012). The California Environmental Protection Agency's Office of Environmental Health Hazard Assessment is requesting the public health goal be set at $0.0007 \mu \mathrm{g} / \mathrm{L}$ for 1,2,3-TCP in drinking water (California Environmental Protection Agency, Office of Environmental Health Hazard Assessment, 2009). Under ambient purge conditions, Method O-4127 (NWQL LS 2020) (Connor and others, 1998) has a RL of $0.12 \mu \mathrm{g} / \mathrm{L}$ for 1,2,3-TCP. Method O-4437 has a MDL of $0.003 \mu \mathrm{g} / \mathrm{L}$, with a RL of $0.006 \mu \mathrm{g} / \mathrm{L}$. Method O-4437 easily achieves the EPA's UCMR $3 \mathrm{MRL}$, but will fall short of the California public health goal.

$\mathrm{DBCP}$ is a regulated drinking water contaminant with a promulgated MCL of $0.2 \mu \mathrm{g} / \mathrm{L}$. EDB is also a regulated drinking water contaminant with a promulgated MCL of $0.05 \mu \mathrm{g} / \mathrm{L}$. Method O-4127 (NWQL LS 2020) (Connor and others, 1998) has a $\mathrm{RL}$ of $0.4 \mu \mathrm{g} / \mathrm{L}$ for DBCP and $0.028 \mu \mathrm{g} / \mathrm{L}$ for EDB. A lower-level method has been available for determination of DBCP and EDB using a hexane extraction with gas chromatography and electron capture detection (EPA Method 504.1 [U.S. Environmental Protection Agency, 1995], NWQL LS 1306), with 
reporting levels for DBCP of $0.03 \mu \mathrm{g} / \mathrm{L}$ and $\mathrm{EDB}$ of $0.018 \mu \mathrm{g} / \mathrm{L}$. Method O-4437 has a MDL for DBCP of $0.010 \mu \mathrm{g} / \mathrm{L}$, with a RL of $0.020 \mu \mathrm{g} / \mathrm{L}$ and a MDL of $0.002 \mu \mathrm{g} / \mathrm{L}$ for EDB, with a RL of $0.004 \mu \mathrm{g} / \mathrm{L}$.

Method O-4437 will easily achieve reporting levels below the EPA MCL requirements.

The California Department of Public Health established a notification level for 1,4-dioxane of $1.0 \mu \mathrm{g} / \mathrm{L}$ in drinking water in 2011 , based upon a revised EPA risk assessment update in 2010 (U.S. Environmental Protection Agency, 2014). Method O-4437 has a MDL of $0.35 \mu \mathrm{g} / \mathrm{L}$, with a RL of $0.7 \mu \mathrm{g} / \mathrm{L}$. 1,4-Dioxane is on the EPA's UCMR 3 list (U.S. Environmental Protection Agency, 2012) with a MRL of $0.07 \mu \mathrm{g} / \mathrm{L}$ using EPA Method 522 (solid phase extraction with GC/MS in SIM mode) (Munch and Grimmett, 2008). Method O-4437 will reliably detect 1,4 -dioxane at $0.7 \mu \mathrm{g} / \mathrm{L}$, but will not achieve the reporting levels of EPA Method 522.

12.1.4. Method Detection Limits and Reporting Levels for Method O-4436-MDLs for the high priority ambient purgeable VOCs for Method O-4436 ranged from $0.007 \mu \mathrm{g} / \mathrm{L}$ for 2,2-dichloro-1,1,1-trifluoroethane to $0.04 \mu \mathrm{g} / \mathrm{L}$ for 1,2,3,4-tetrahydronaphthalene, and 1,3-butadiene using the EPA procedure (U.S. Environmental Protection Agency, 2002). Four semivolatile compounds (2-chloronaphthalene, 1,2-dimethylnaphthalene, 1,6-dimethylnaphalene, and 2,6-di-tert-butyl phenol) had initial MDLs ranging from 0.08 to $0.1 \mu \mathrm{g} / \mathrm{L}$. After acquiring data for 18 months, a more realistic MDL for the dimethylnaphthalene isomers would be 0.5 to $1 \mu \mathrm{g} / \mathrm{L}$. All four semivolatile compounds were deleted from the method because of poor performance and carryover issues. The reporting level is defined as two times the MDL (table 12) for the high priority ambient purgeable VOCs.

MDLs for the ambient purgeable VOCs retained from O-4127 (NWQL LS 2020) ranged from $0.005 \mu \mathrm{g} / \mathrm{L}$ for 1,1,2-trichloroethane to $0.06 \mu \mathrm{g} / \mathrm{L}$ for bromoform using the EPA procedure. MDLs for the ambient purgeable VOCs in Method O-4127 (NWQL LS 2020) (Connor and others, 1998) have been collected since 1998, using a modified EPA procedure (Childress and others, 1999). Method O-4127 MDLs will be referred to as historical MDLs. The historical MDLs ranged from $0.009 \mu \mathrm{g} / \mathrm{L}$ for trans1,2-dichloroethene to $0.1 \mu \mathrm{g} / \mathrm{L}$ for bromomethane.

A 1:1 comparison of Method O-4436 MDLs and Method O-4127 historical MDLs in figure 4 (table 12, data) shows that the majority of the compounds (29 of 35) have lower MDLs using Method O-4436. Some of the difference in MDLs may be because of the internal standard compound change. However, it may

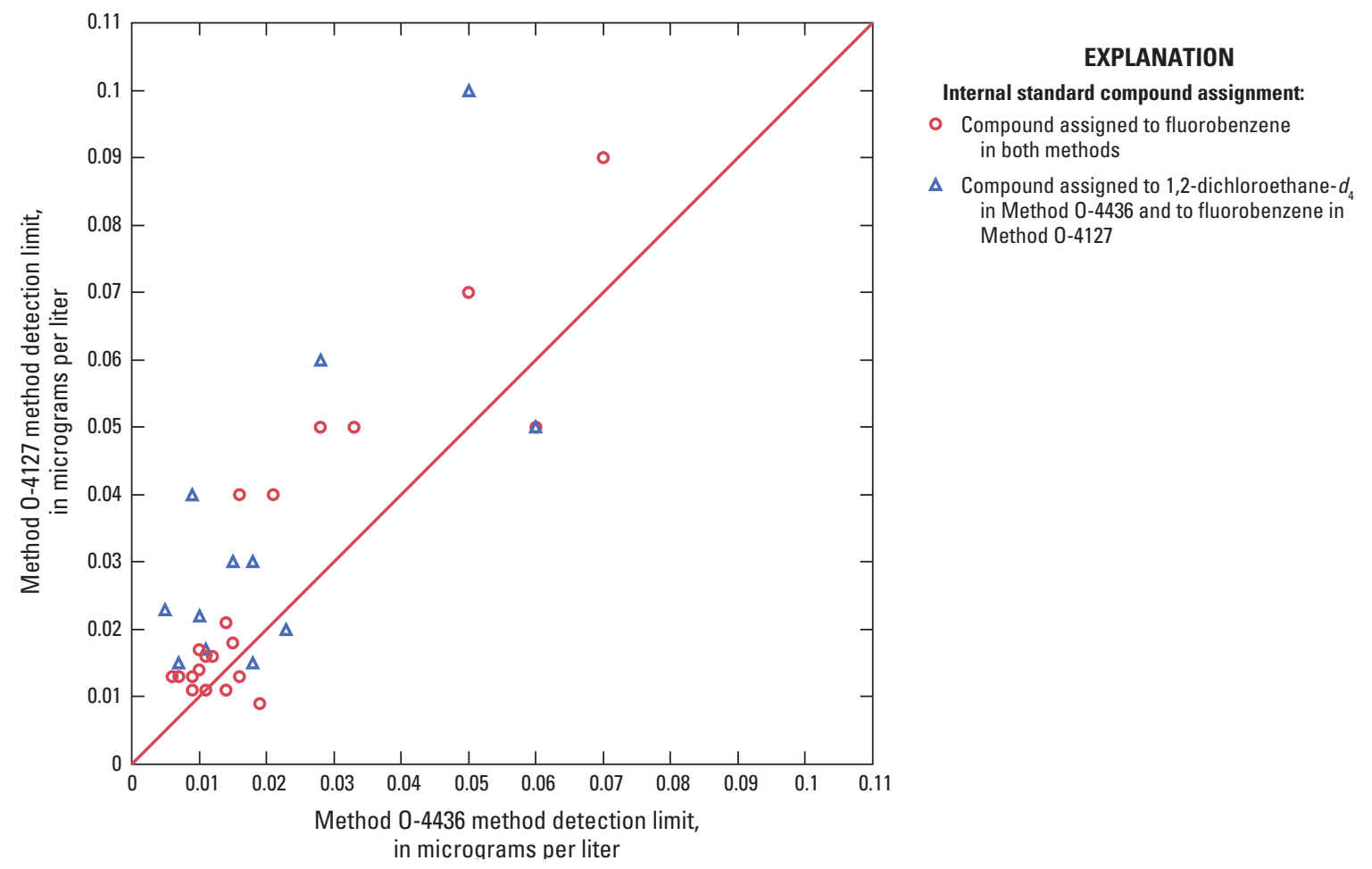

Figure 4. Comparison of method detection limits for ambient purgeable Methods 0-4436 and 0-4127. 
be that more of the difference in MDLs between the methods is attributable to the difference in the collection of data used to calculate the MDLs. The MDLs for Method O-4127 were calculated based on replicate spikes analyzed over 12 months by multiple operators and multiple instruments. The procedure was repeated and re-evaluated on a yearly basis. The MDLs for Method O-4436 were collected on one instrument, by one analyst over 2 weeks. The historical MDLs were selected for the ambient purgeable VOCs that were retained from Method $\mathrm{O}-4127$. The reporting level is defined as two times the historical MDL for the ambient purgeable VOCs retained from Method O-4127 (table 12).

12.1.5. Blank-Limited Compounds and Minimum Reporting Levels, Method 0-4436 - Three of the compounds from Method O-4127 (NWQL LS 2020) (Connor and others, 1998) have been historically designated as blank-limited compounds and will be reported as blank-limited compounds on Method O-4436. Methylene chloride, chloromethane, and toluene are reported with MRLs of $<0.04 \mu \mathrm{g} / \mathrm{L},<0.1 \mu \mathrm{g} / \mathrm{L}$, and $<0.02 \mu \mathrm{g} / \mathrm{L}$, respectively. MDLs are not calculated for these compounds. Methylene chloride is a common laboratory solvent and is often detected at low concentrations in laboratory environments. Methylene chloride and chloromethane have been detected in the concentrated $\mathrm{HCl}$ purchased from the vendor at low concentrations. Toluene is also a common solvent and a component of gasoline.

\subsection{Bias and Variability}

Bias and variability were evaluated by analyzing a minimum of seven fortified replicates in VBW, groundwater samples, and surface water samples. Samples at two concentrations were analyzed for each compound. The groundwater sample was from an urban production well (USAWR-12) in Corona, Calif., sampled June 15, 2011, at 09:30 am, with a dissolved organic carbon content of 1.41 milligram per liter $(\mathrm{mg} / \mathrm{L})$. The surface water sample was from Hillsboro Canal, sampled June 21, 2011, at 12:00 pm in Shawano, Fla., with a dissolved organic carbon content of $19.8 \mathrm{mg} / \mathrm{L}$. The groundwater and surface water samples were collected in 1-L amber bottles and stored at $4 \pm 2{ }^{\circ} \mathrm{C}$. Then, $50 \mathrm{~mL}$ of water was fortified with method compounds, preserved to $\mathrm{pH} 2$ with $\mathrm{HCl}$ (1:1), and transferred to a 40-mL VOC vial for analysis. An unfortified sample of each water matrix was also analyzed to determine if any method compounds were detectable. Two or three fortified replicates were prepared in each matrix, at two concentration levels, and analyzed. This procedure was repeated on two other occasions over 2 weeks until a minimum of seven fortified replicates was obtained for each matrix and concentration.
12.2.1. Result of Bias and Variability for Method O-4437Methyl oxirane and oxirane degraded quickly in the presence of acidic aqueous solutions, and were subsequently eliminated from the method because of incompatibility with the $\mathrm{HCl}(1: 1)$ preservative. Mean recoveries in VBW, groundwater, and surface water for methyl oxirane range from 2 to 46 percent, with RSDs from 123 to 250 percent. Mean recoveries for oxirane range from 35 to 55 percent, with RSDs from 28 to 78 percent.

Mean recoveries for remaining heat purgeable VOCs in all three matrices ranged from 79 to 122 percent with RSDs $\leq 30$ percent, except for hexachlorocyclopentadiene and 1-octanol (table 13). Mean recoveries for hexachlorocyclopentadiene in all three matrices ranged from 122 to 152 percent with RSDs from 16 to 42 percent. Mean recoveries for 1-octanol ranged from 86 to 133 percent, with RSDs $\leq 16$ percent. The 133 percent recovery was in the surface water for the high-level spike. The recoveries in the other two matrices ranged from 86 to 120 percent (table 13).

12.2.2. Results of Bias and Variability for Method O-4436Mean recoveries for the ambient purgeable VOCs met data quality objectives for all compounds, with the exception of 2,6-di-tert-butyl phenol and chloromethane. Mean percent recovery for 2,6-di-tert-butyl phenol was 136 percent and chloromethane was 138 percent in the $5 \mu \mathrm{g} / \mathrm{L}$ surface water spike (table 14). Because of poor performance, 2,6-di-tert-butyl phenol was removed from the method in May 2014. Chloromethane, however, was not eliminated from the method, but is reported with an "E" remark code, indicating an estimated concentration.

\subsection{Holding Time Study Description}

A holding time study was conducted for 28 days for each method to determine estimated holding times for both heat and ambient purgeable VOCs. Five replicate samples were prepared in VBW; adjusted to $\mathrm{pH} 2$ with $\mathrm{HCl}$ (1:1); fortified at concentrations ranging from 0.064 to $10 \mu \mathrm{g} / \mathrm{L}$, depending on the compound; transferred to $40-\mathrm{mL}$ VOC vials; and stored at $4 \pm 2{ }^{\circ} \mathrm{C}$ for $0,7,14$, and 28 days. An unspiked sample was prepared, stored, and analyzed with the fortified samples. A fresh calibration curve was prepared each day.

Preservation with $\mathrm{HCl}(1: 1)$ has been used for the O-4127 (NWQL LS 2020) ambient purgeable VOCs since 1998 (Connor and others, 1998). O-4024 (NWQL LS 4024) has not required sample preservation for determination of four gasoline oxygenates and four degradation products (Rose and Sandstrom, 2003).

A 14-day holding time has been used by EPA for many years and allows adequate time for laboratory processing. Estimated holding times were calculated, based on the ASTM procedure D-4841-88 (ASTM International, 2001). The ASTM 
procedure estimates a holding time based on a tolerable range of deviation. The holding time study results are considered acceptable if the mean recovery is within 70-130 percent on day 14 . The actual data for day 14 will be used to override the ASTM estimated holding time when the ASTM data predict unacceptable holding times and actual results show acceptable recovery and variability on day 14 . Preservation studies using these methods reveal that some VOCs are stable for much longer periods. Love and others (1998) provide stability data for a 216-day study for the VOCs in Method O-4127 (NWQL LS 2020).

12.3.1. Discussion and Results of Holding Time Study, Method O-4437-During the initial method development for the heat purgeable method, the protocol for Method O-4024 (NWQL LS 4024) (Rose and Sandstrom, 2003) was followed. It was not known if sample preservation would be required or not for Method O-4437 (NWQL LS 4437). The holding time study in Rose and Sandstrom (2003) prepared fortified sample replicates in surface water with no preservative and in VBW adjusted to $\mathrm{pH} 2$ with $\mathrm{HCl}(1: 1)$. The same procedure was followed in this study. Surface water from Boulder Creek at 75th Street, Boulder, Colo., was sampled and fortified with method compounds without preservative. VBW fortified samples were adjusted to $\mathrm{pH} 2$ with $\mathrm{HCl}$ (1:1). The calibration standards were not adjusted to $\mathrm{pH} 2$ since preservation was not required for the samples in Method O-4024. Analyses of the day zero fortified samples demonstrated that the unpreserved samples in the Boulder Creek surface water were quickly degrading during the course of the instrument batch run for several of the compounds. To be consistent with the day zero analyses, calibration curves for days 7, 14, and 28 were prepared in VBW without the $\mathrm{HCl}(1: 1)$ added.

Mean percent recoveries were calculated for day zero analyses based on the fortified amount added to the samples. 2,6-Dimethyl-4-heptanone had a mean recovery on day zero of 73 percent, quantitated against the calibration standards made without the preservative. Mean recovery on day 14 for 2,6-dimethyl4-heptanone decreased to 67 percent. The lower recoveries for 2,6-dimethyl-4-heptanone are considered a result of the absence of the $\mathrm{HCl}(1: 1)$ in the calibration standards rather than because of losses.

Methyl oxirane degraded immediately in the VBW samples preserved with $\mathrm{HCl}(1: 1)$ to $\mathrm{pH} 2$. The mean recovery for oxirane on day zero was 22 percent in the preserved VBW samples. By day 7 oxirane was not detected in any of the preserved VBW samples. 2-Chloromethyl oxirane had a mean recovery of 82 percent on day zero in the preserved VBW samples. By day 7 mean recovery was 50 percent; by day 14 , recovery was 44 percent. Methyl oxirane, oxirane, and 2-chloromethyl oxirane were deleted from the method because of incompatibility with the $\mathrm{HCl}$ (1:1) preservative (fig. 5).
The remaining heat purgeable VOCs for Method O-4437 performed within data quality objectives without significant losses. Mean recoveries on day zero ranged from 78 to 125 percent, declining slightly from 73 to 115 percent by day 14 . Percent recovery of hexachlorocyclopentadiene increased from 86 percent on day zero to 112 percent on day 14 . This compound has displayed considerable variability. Hexachlorocyclopentadiene has a complex degradation profile, undergoing photolysis, hydrolysis, and volatilization. The variability exhibited for this compound is probably attributable to more than merely method variation.

Table 15 presents a summary of recovery and variability. Figure 5 shows a graphical summary of the holding time study for each compound.

The calculated holding time using the ASTM procedure exceeded 14 days for 36 of the 38 heat purgeable VOCs tested (table 16). The calculated ASTM holding time for acrolein was 7 days at $\mathrm{pH}$ 2. EPA Method 603 (U.S. Environmental Protection Agency, 1984) requires a sample $\mathrm{pH}$ of $4-5$ for analysis of acrolein. Recovery for acrolein was within the data quality objectives of 70-130 percent, decreasing from 125 percent on day zero to 114 percent on day 14 . Acrolein was retained throughout the method validation and initial implementation. Upon review of long-term data, however, acrolein was subsequently eliminated from the method on April 1, 2016 because of instability and inconsistent performance. The calculated ASTM holding time for propyl acetate was 10 days. Recovery for propyl acetate decreased from 92 percent on day zero to 82 percent by day 14 (table 15; fig. 5). Based upon the recoveries on day 14, propyl acetate will be assigned a 14-day holding time.

Sample preservation to $\mathrm{pH} 2$ with $\mathrm{HCl}(1: 1)$ and a maximum 14-day holding time is recommended to preserve the compounds for the heat purgeable method.

12.3.2. Discussion and Results of Holding Time Study, Method O-4436-With the exception of hexane and benzyl chloride, all ambient purgeable VOCs in Method O-4436 (NWQL LS 4436) performed within data quality objectives without significant losses. Mean recoveries for the ambient purgeable VOCs on day 14 ranged from 77 to 105 percent, with standard deviations of $\leq 7$ percent. Mean recovery for hexane was 53 percent on day 14 with a standard deviation of 2 percent; mean recovery for benzyl chloride was 33 percent on day 14 with a standard deviation of 3 percent.

Table 17 presents a summary of recovery and variability. Figure 6 shows a graphical summary of the holding time study for each compound. 

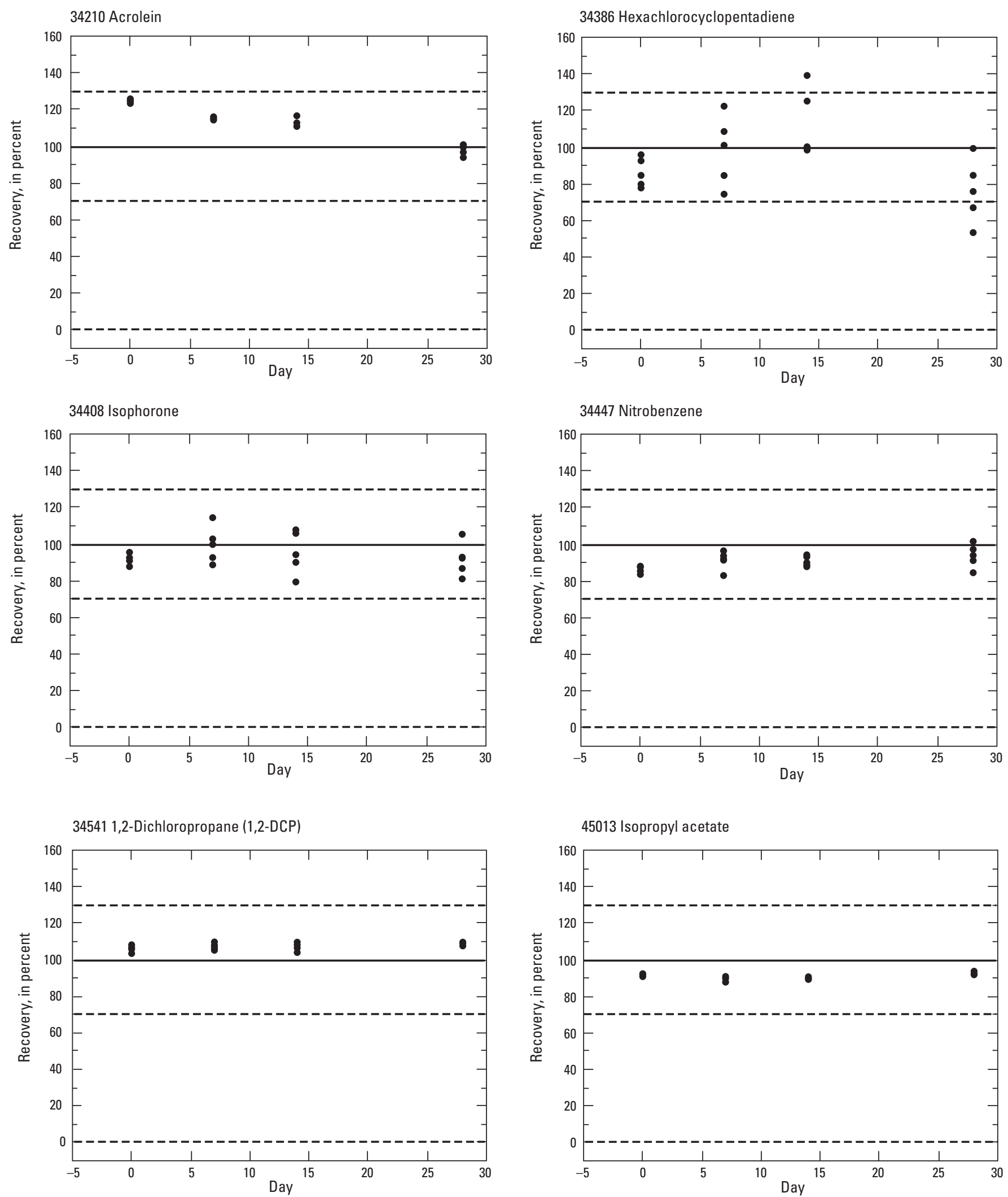

Figure 5. Summary of a 28-day holding time study for heat purgeable volatile organic compounds. Twenty fortified replicate spikes were prepared in volatile blank water, preserved to $\mathrm{pH} 2$ with hydrochloric acid (1:1), and stored at 4 plus or minus 2 degrees Celsius. Five replicate spikes were determined by Method 0-4437 on days 0, 7, 14, and 28. The dashed lines at 70 and 130 percent represent the acceptable limits used to evaluate stability over time. Graphs are sorted in parameter code order. 

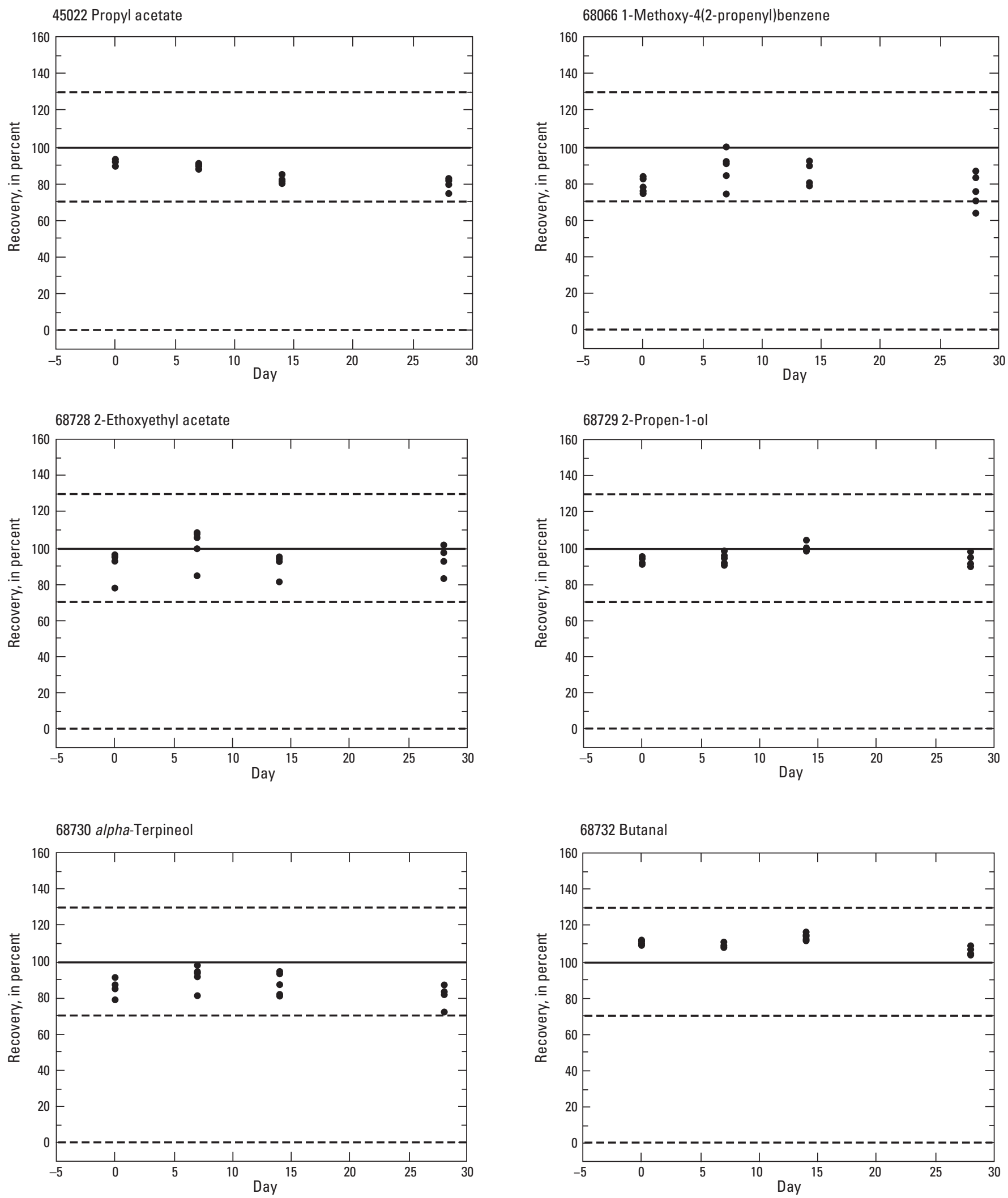

Figure 5. Summary of a 28-day holding time study for heat purgeable volatile organic compounds. Twenty fortified replicate spikes were prepared in volatile blank water, preserved to $\mathrm{pH} 2$ with hydrochloric acid (1:1), and stored at 4 plus or minus 2 degrees Celsius. Five replicate spikes were determined by Method 0-4437 on days 0, 7, 14, and 28. The dashed lines at 70 and 130 percent represent the acceptable limits used to evaluate stability over time. Graphs are sorted in parameter code order.-Continued 

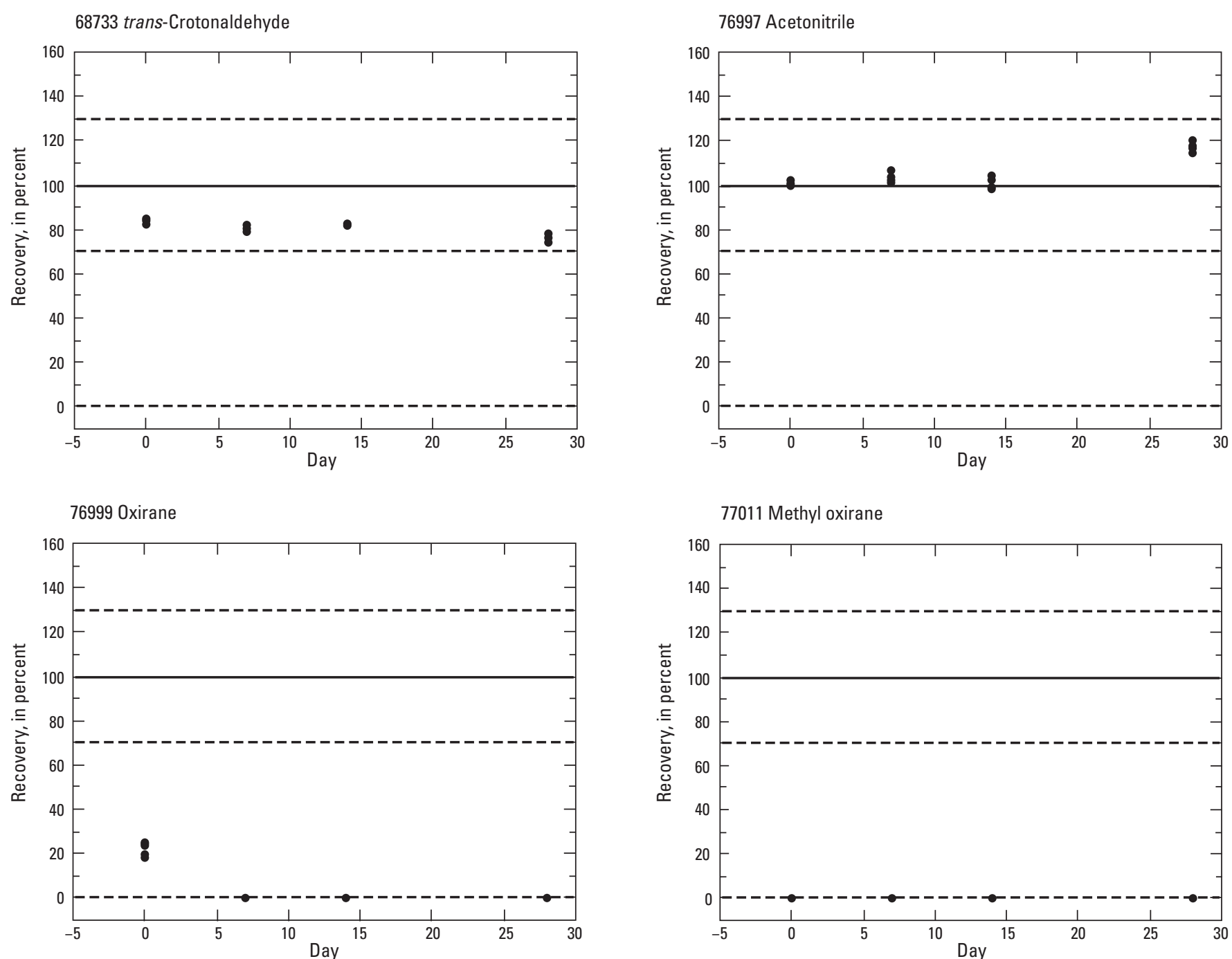

77011 Methyl oxirane

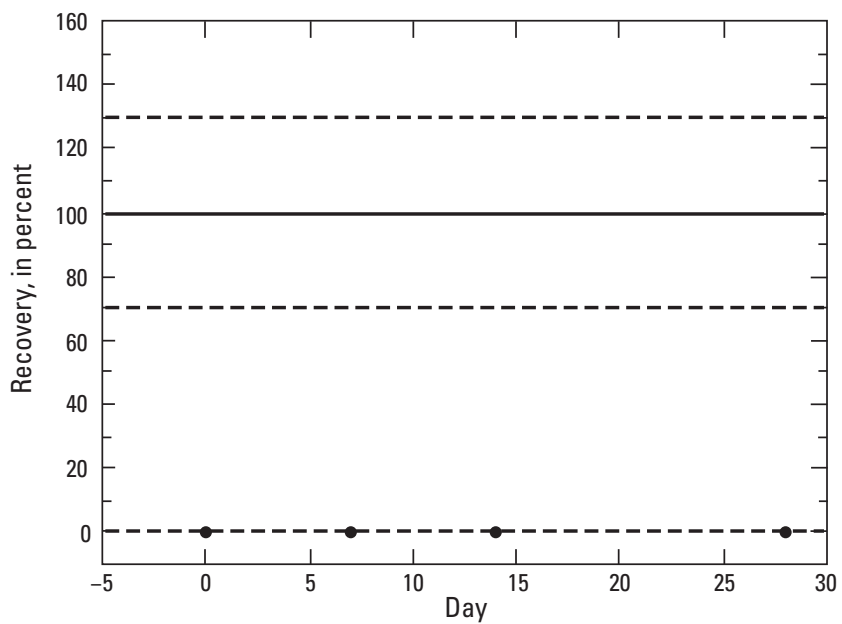

77015 Isopropyl alcohol

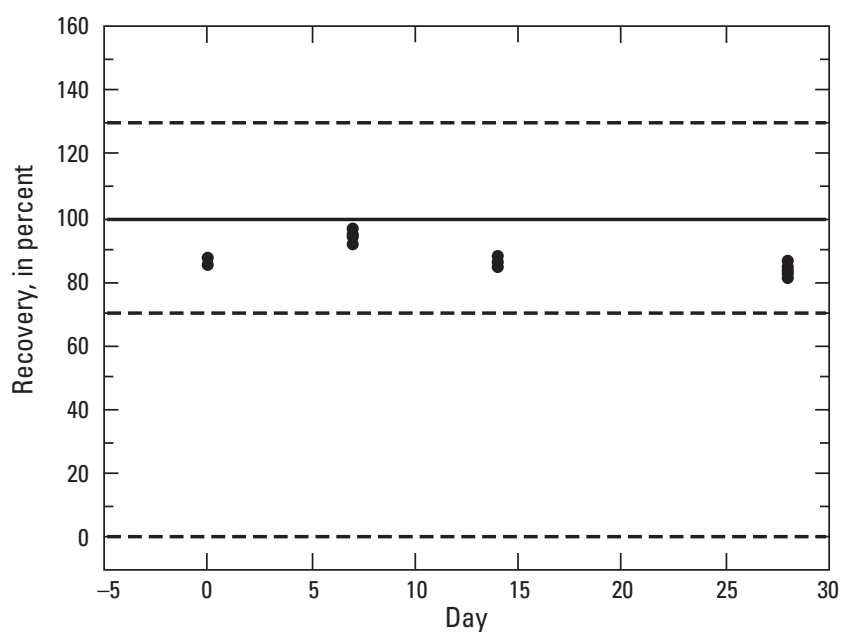

77032 Methyl acetate

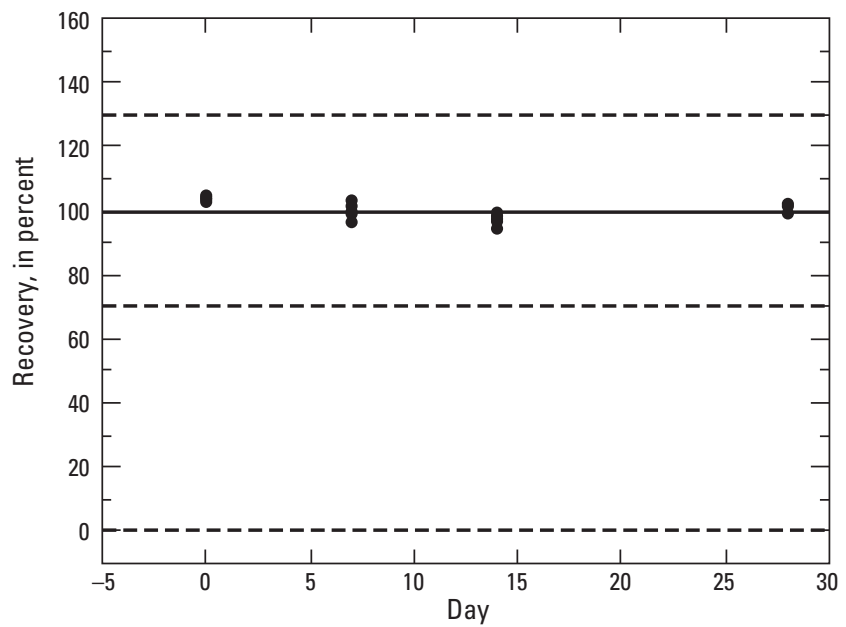

Figure 5. Summary of a 28-day holding time study for heat purgeable volatile organic compounds. Twenty fortified replicate spikes were prepared in volatile blank water, preserved to $\mathrm{pH} 2$ with hydrochloric acid (1:1), and stored at 4 plus or minus 2 degrees Celsius. Five replicate spikes were determined by Method 0-4437 on days 0, 7, 14, and 28. The dashed lines at 70 and 130 percent represent the acceptable limits used to evaluate stability over time. Graphs are sorted in parameter code order.-Continued 

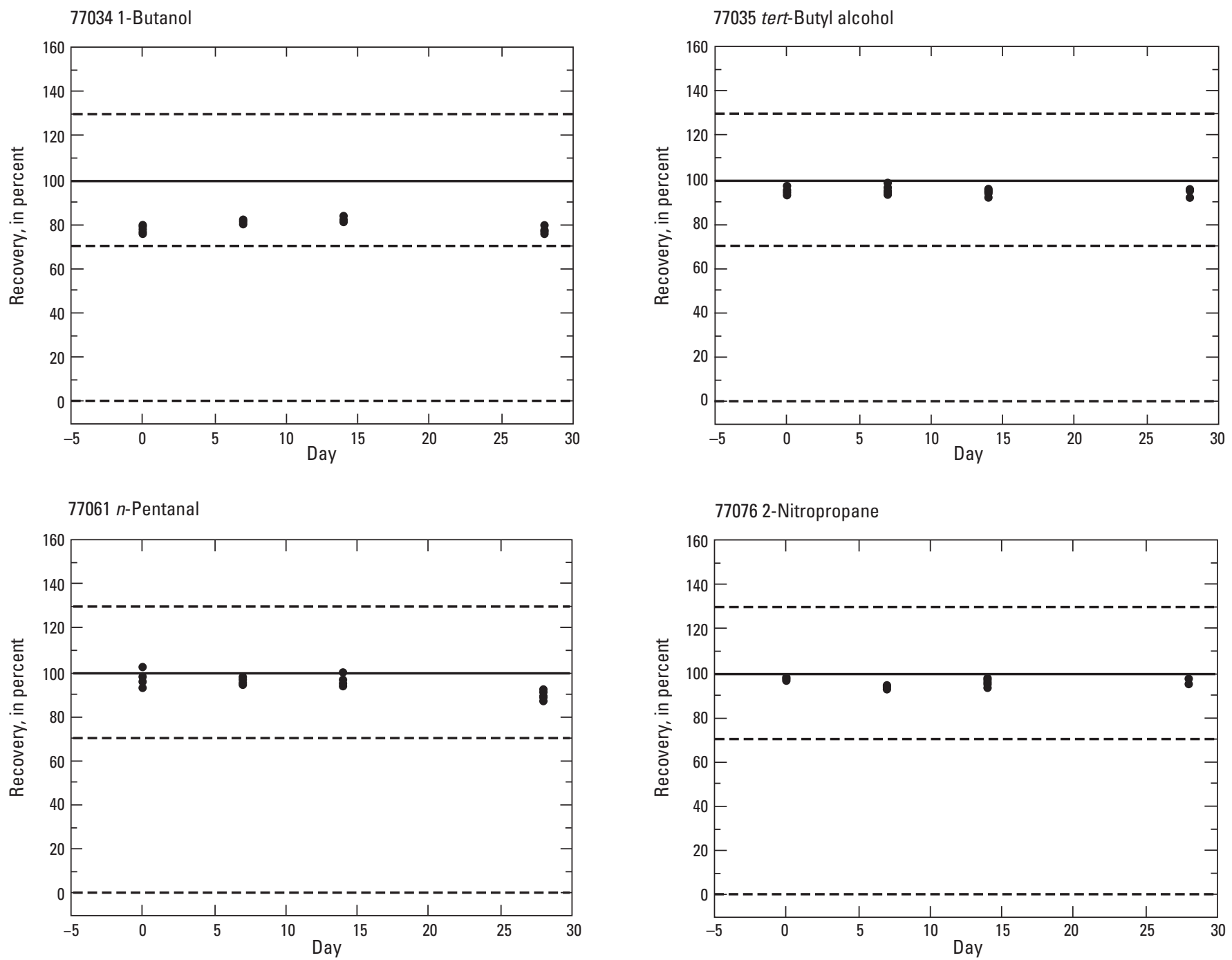

77084 2-Chloromethyloxirane
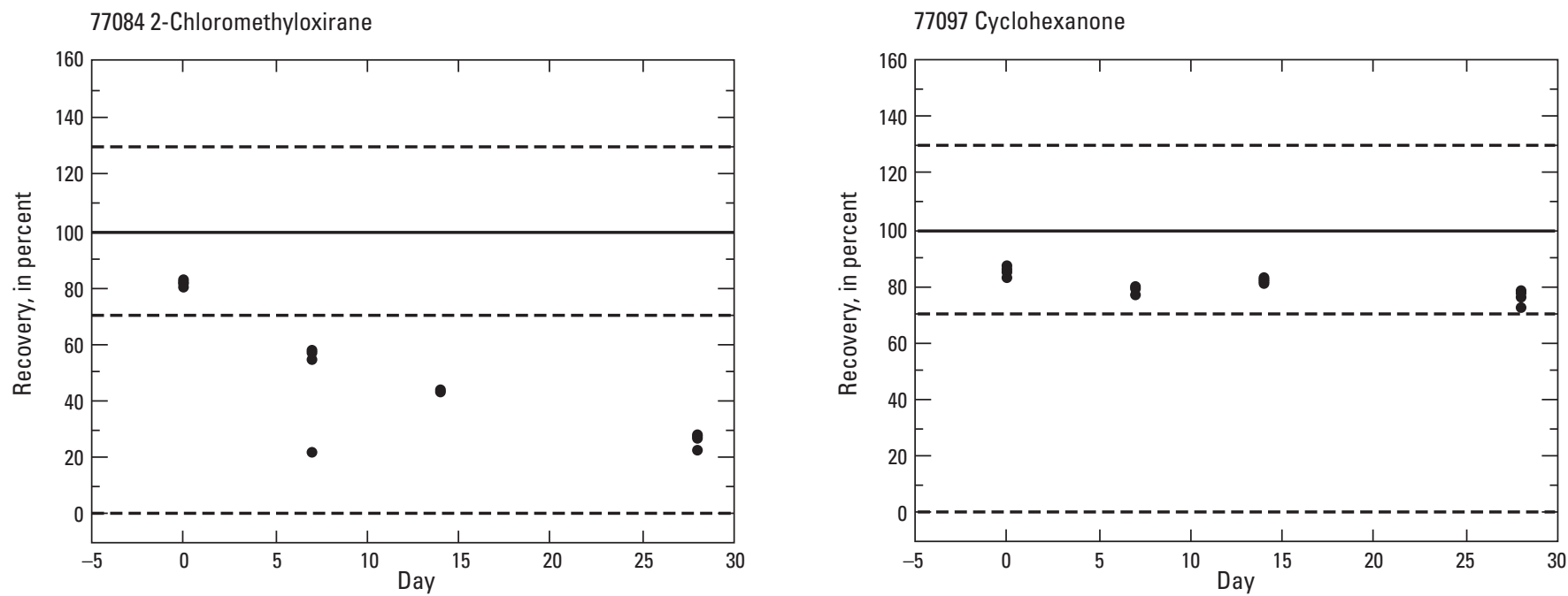

Figure 5. Summary of a 28-day holding time study for heat purgeable volatile organic compounds. Twenty fortified replicate spikes were prepared in volatile blank water, preserved to $\mathrm{pH} 2$ with hydrochloric acid (1:1), and stored at 4 plus or minus 2 degrees Celsius. Five replicate spikes were determined by Method 0-4437 on days 0, 7, 14, and 28. The dashed lines at 70 and 130 percent represent the acceptable limits used to evaluate stability over time. Graphs are sorted in parameter code order.-Continued 
77113 4-Methyl-2-pentanol

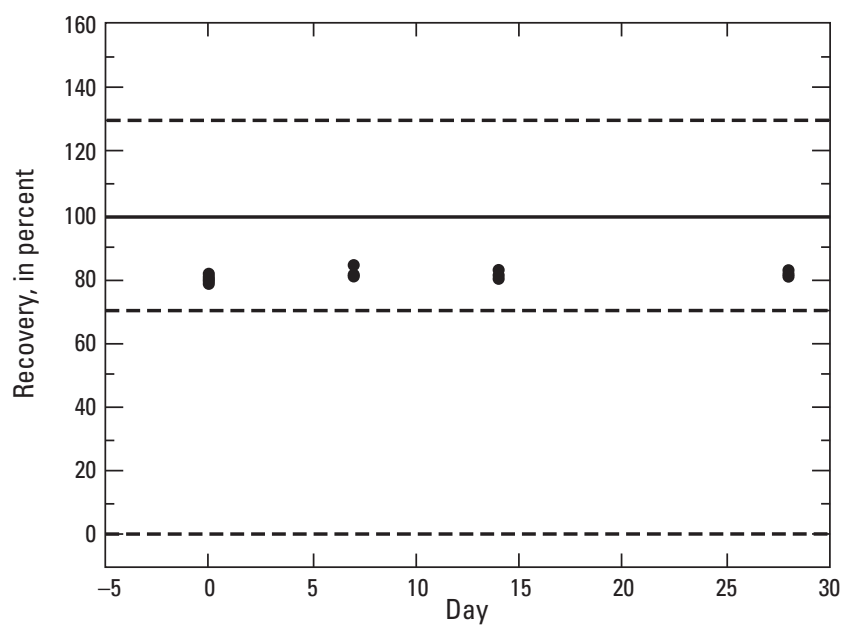

77201 2-Methylpropyl acetate

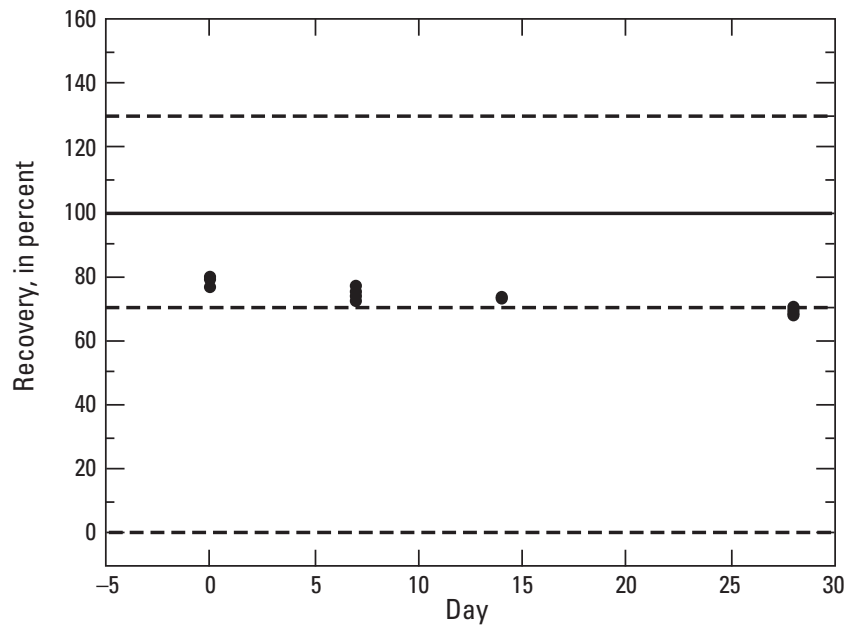

77311 2-Ethyl-1-hexanol

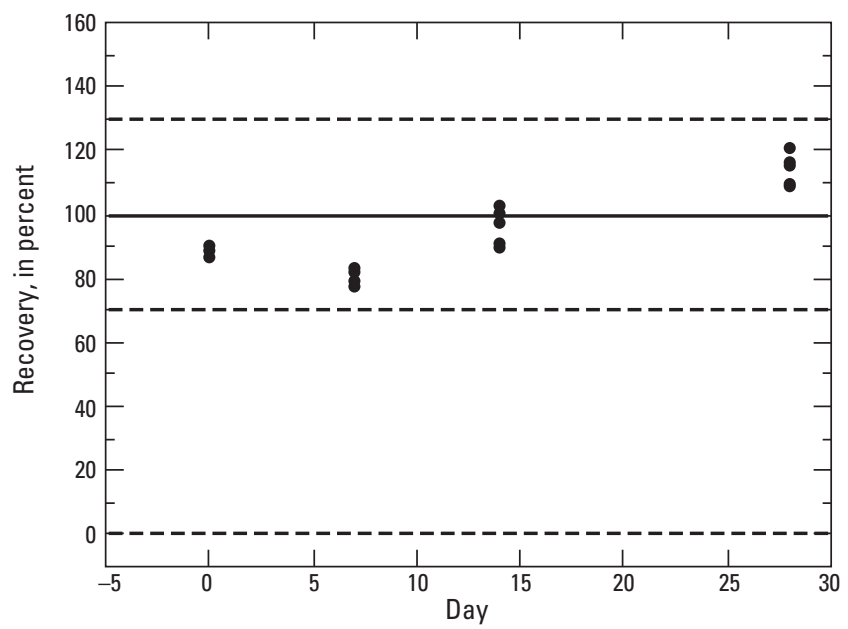

77179 5-Methyl-2-hexanone

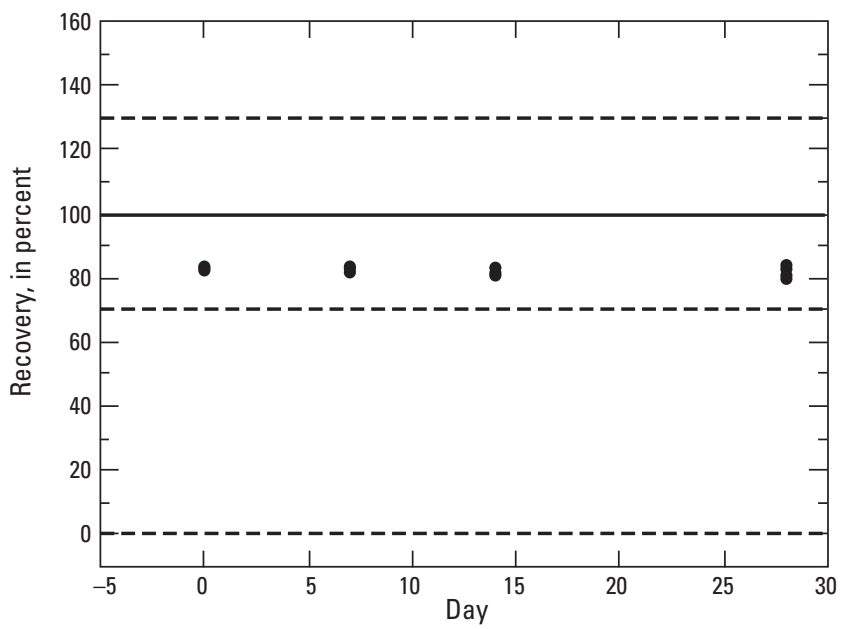

77310 1-0ctanol

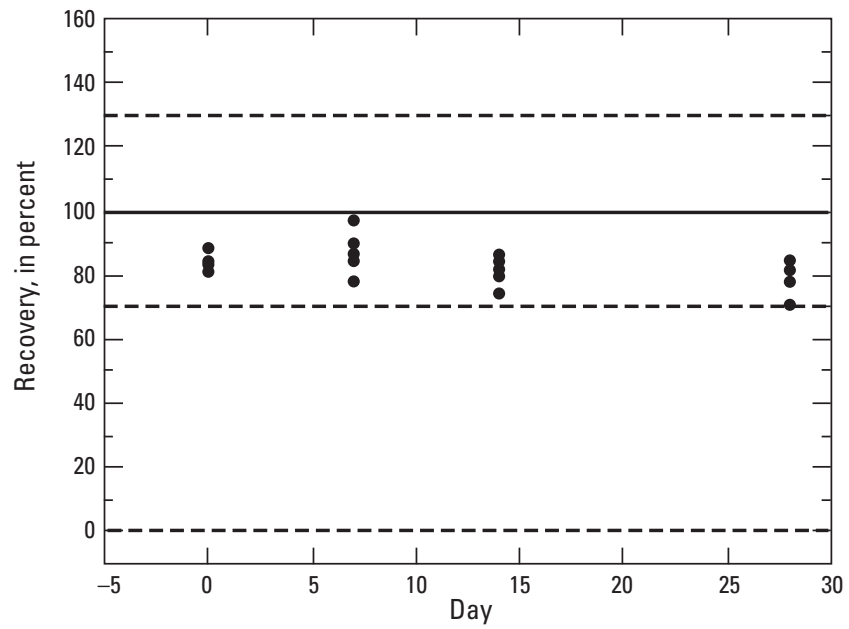

77419 2,6-Dimethyl-4-heptanone

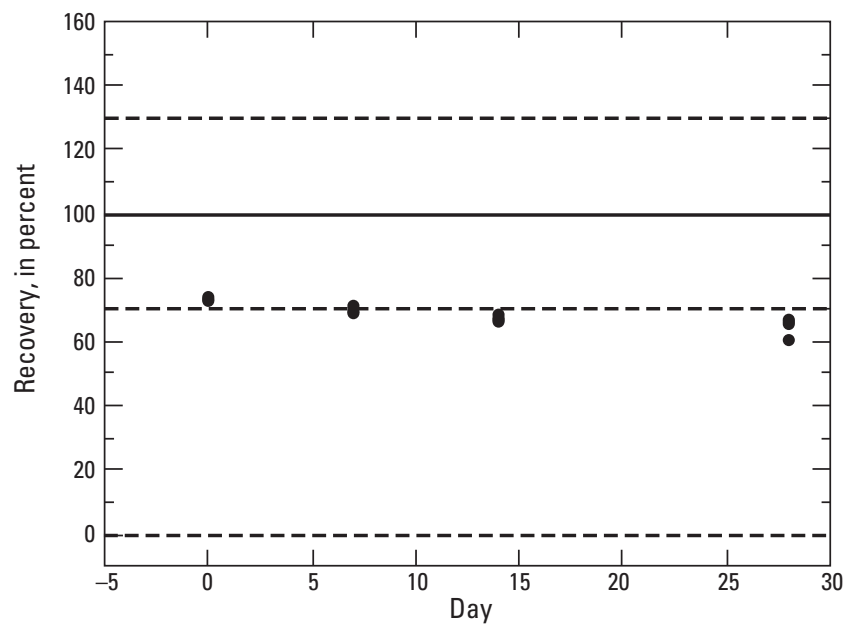

Figure 5. Summary of a 28-day holding time study for heat purgeable volatile organic compounds. Twenty fortified replicate spikes were prepared in volatile blank water, preserved to $\mathrm{pH} 2$ with hydrochloric acid (1:1), and stored at 4 plus or minus 2 degrees Celsius. Five replicate spikes were determined by Method 0-4437 on days 0, 7, 14, and 28. The dashed lines at 70 and 130 percent represent the acceptable limits used to evaluate stability over time. Graphs are sorted in parameter code order.-Continued 
77443 1,2,3-Trichloropropane (1,2,3-TCP)

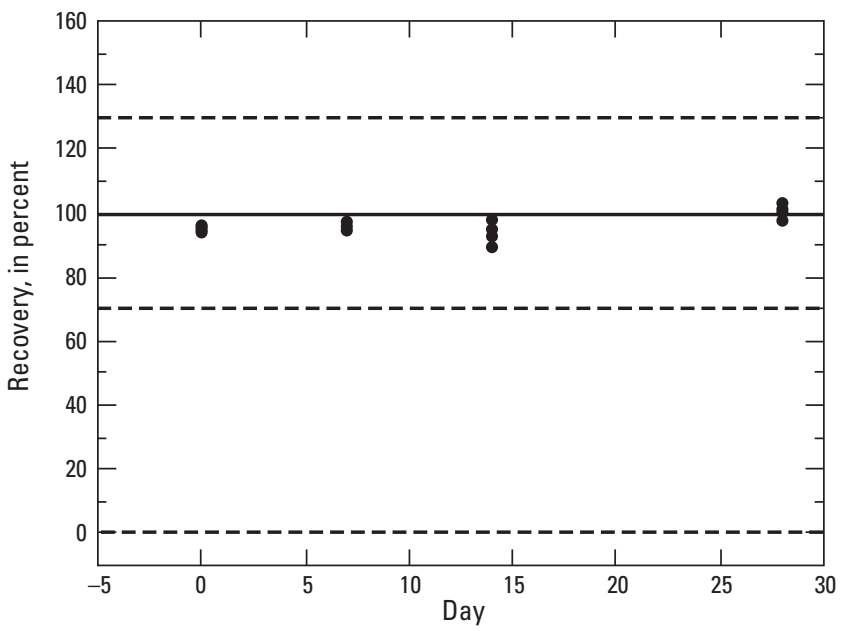

77651 1,2-Dibromoethane (EDB)

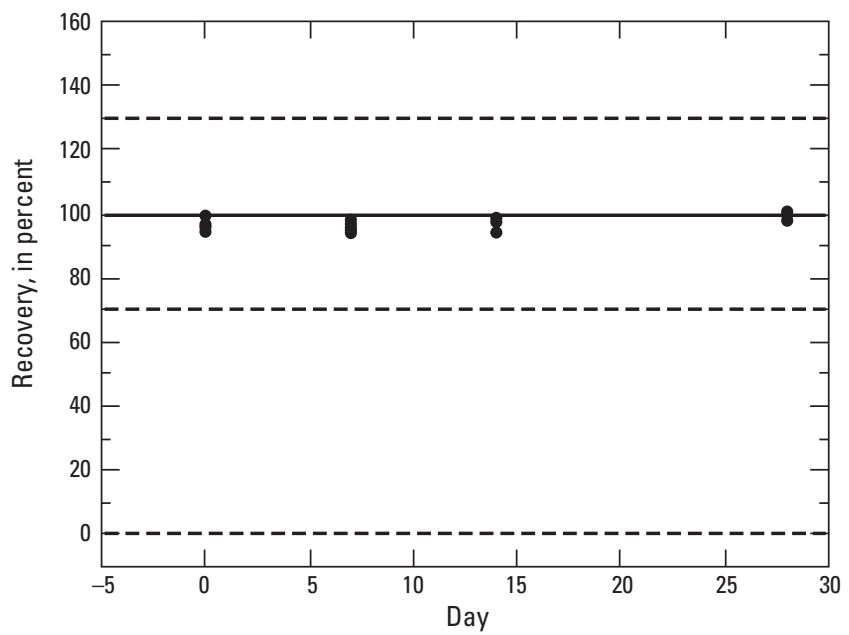

$78200 \mathrm{~N}-$ Nitrosodiethylamine

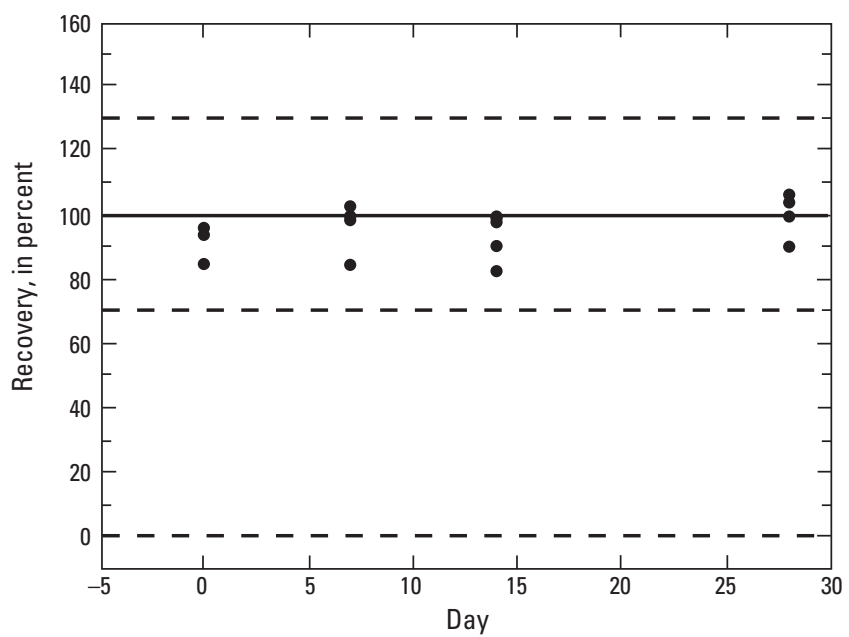

77548 Chloropicrin

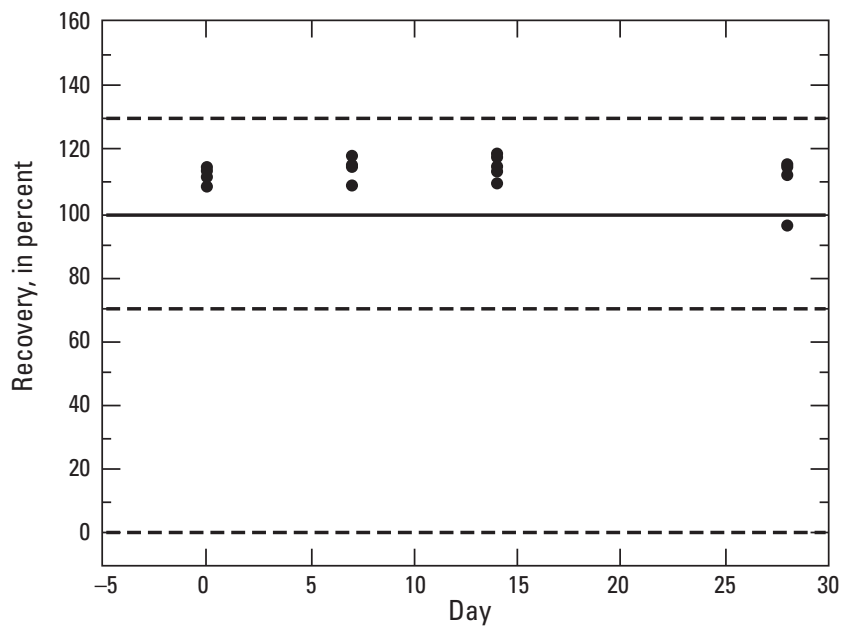

78032 tert-Butyl methyl ether (MTBE)

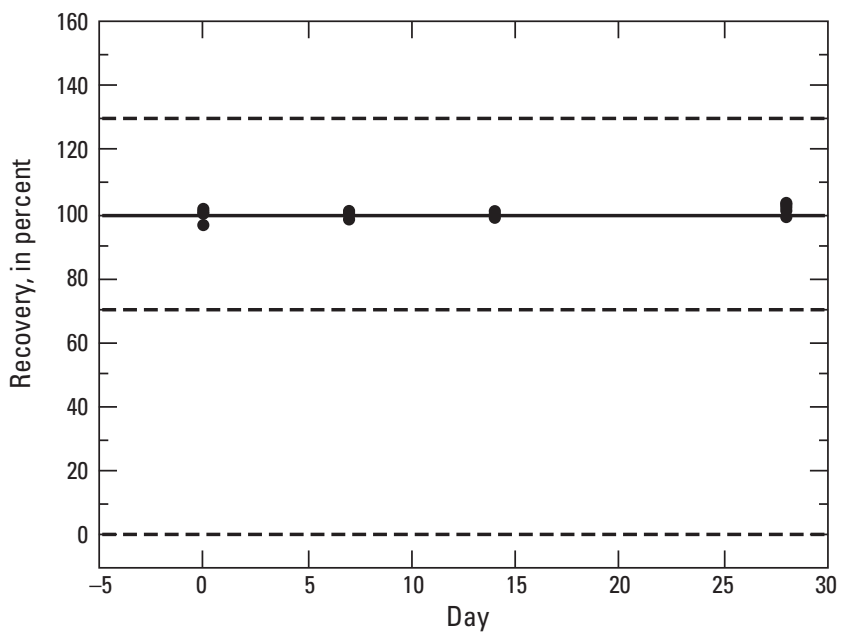

80336 1,1-Dichloro-2-propanone

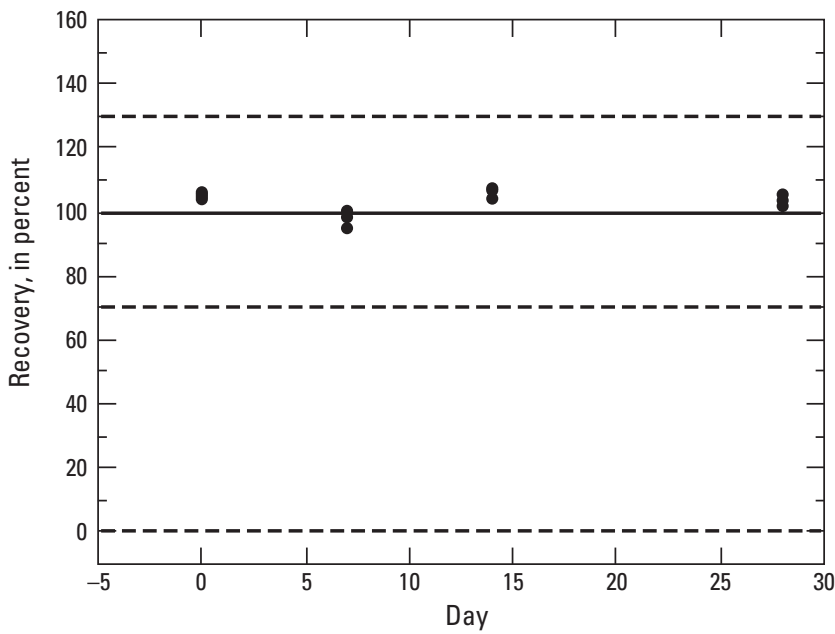

Figure 5. Summary of a 28-day holding time study for heat purgeable volatile organic compounds. Twenty fortified replicate spikes were prepared in volatile blank water, preserved to $\mathrm{pH} 2$ with hydrochloric acid (1:1), and stored at 4 plus or minus 2 degrees Celsius. Five replicate spikes were determined by Method 0-4437 on days 0, 7, 14, and 28. The dashed lines at 70 and 130 percent represent the acceptable limits used to evaluate stability over time. Graphs are sorted in parameter code order.-Continued 

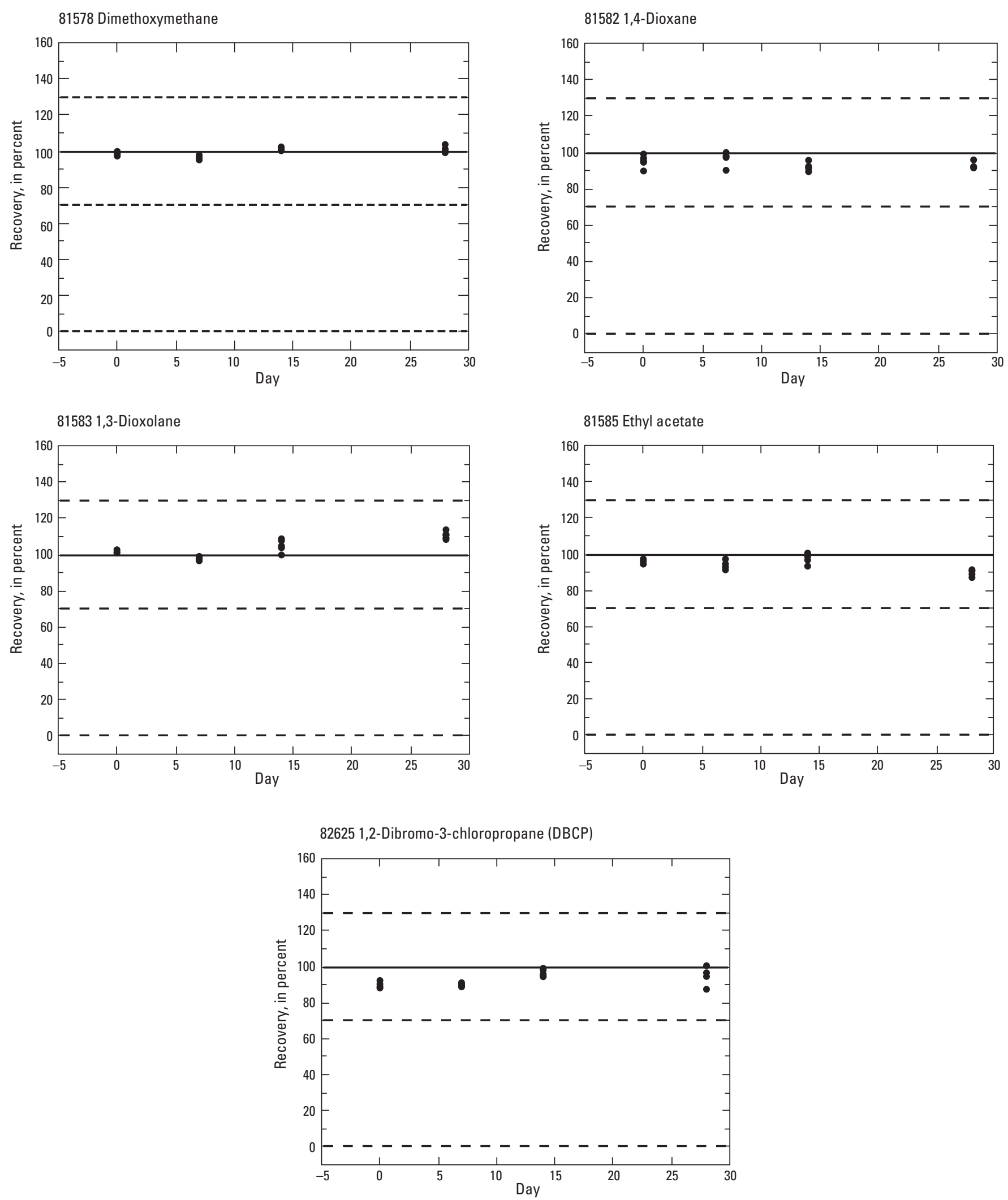

Figure 5. Summary of a 28-day holding time study for heat purgeable volatile organic compounds. Twenty fortified replicate spikes were prepared in volatile blank water, preserved to $\mathrm{pH} 2$ with hydrochloric acid (1:1), and stored at 4 plus or minus 2 degrees Celsius. Five replicate spikes were determined by Method 0-4437 on days 0, 7, 14, and 28. The dashed lines at 70 and 130 percent represent the acceptable limits used to evaluate stability over time. Graphs are sorted in parameter code order.-Continued 
32101 Bromodichloromethane

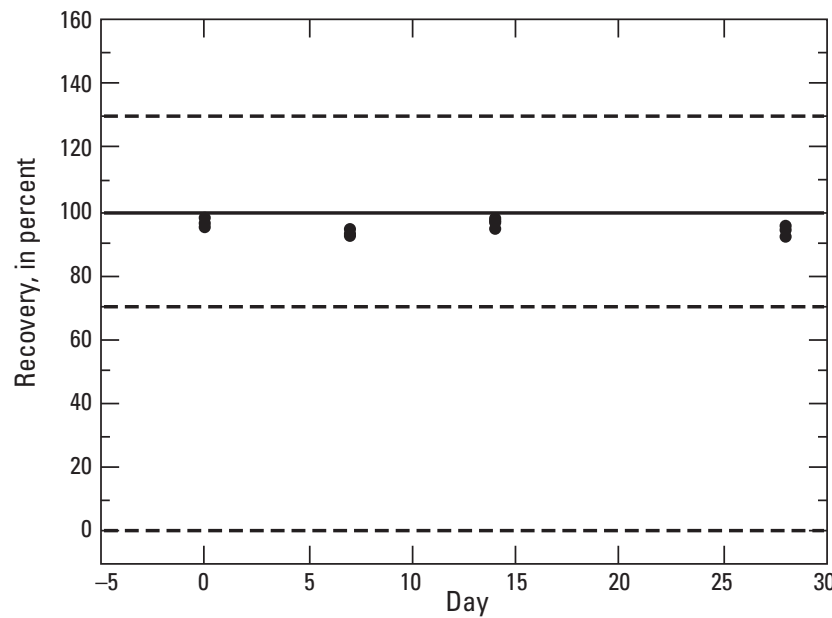

32103 1,2-Dichloroethane

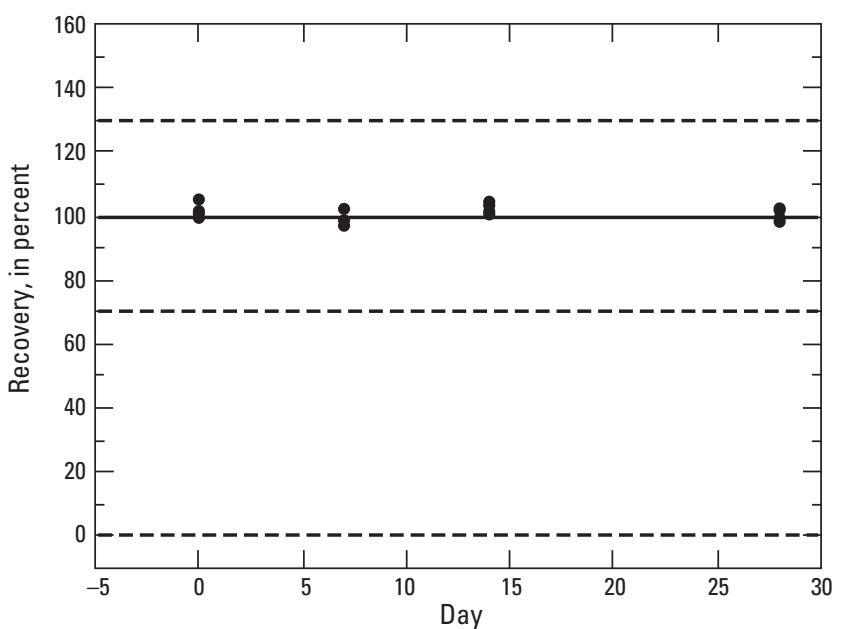

32105 Dibromochloromethane

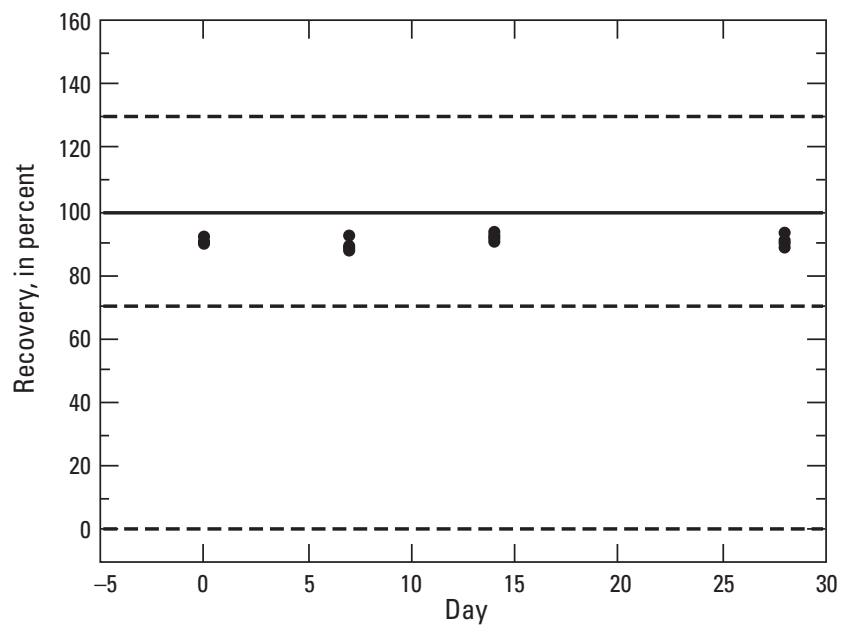

32102 Tetrachloromethane

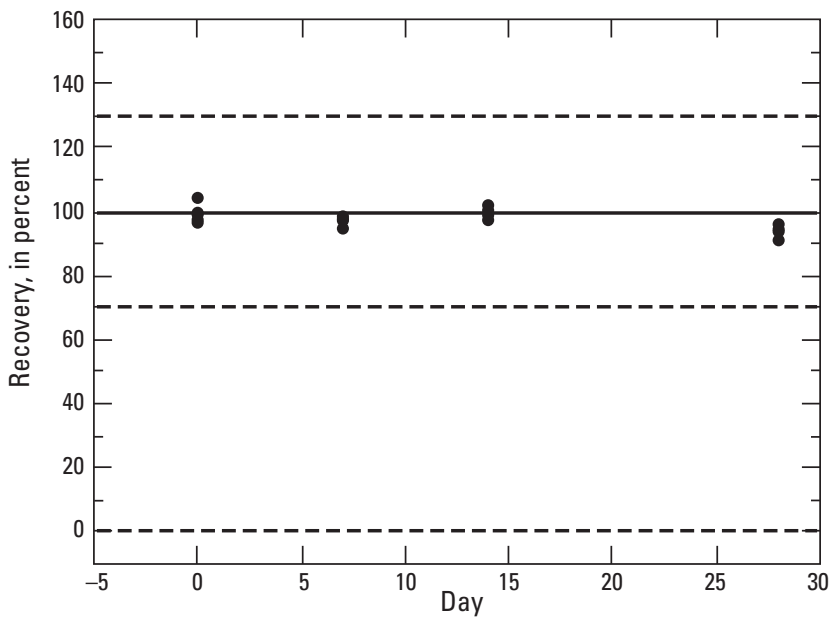

32104 Bromoform

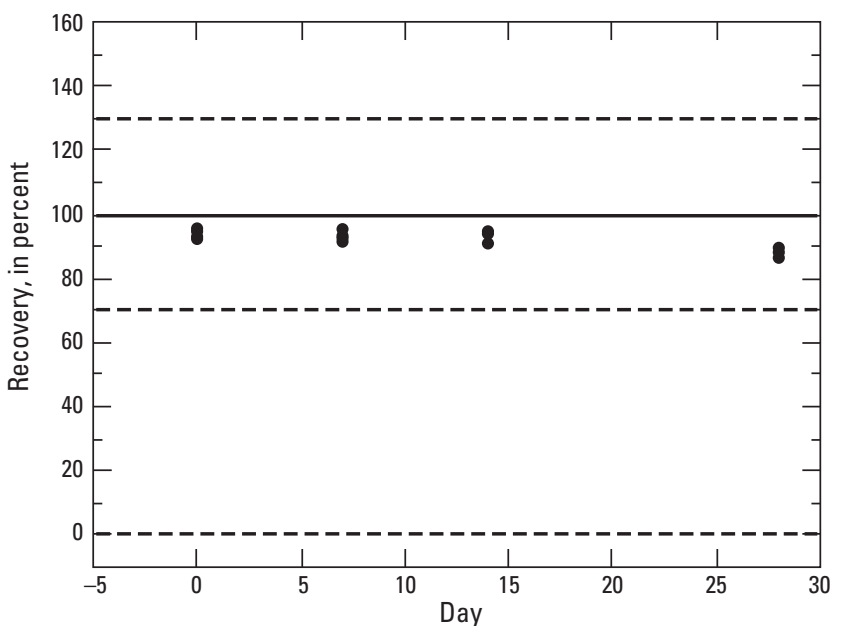

32106 Chloroform

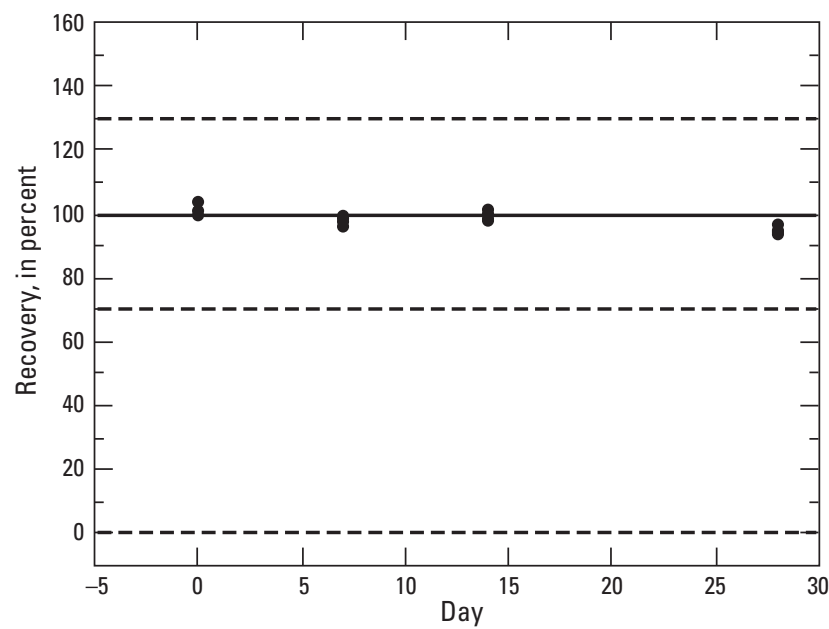

Figure 6. Summary of a 28-day holding time study for ambient purgeable volatile organic compounds. Twenty fortified replicate spikes were prepared in volatile blank water, preserved to $\mathrm{pH} 2$ with hydrochloric acid (1:1), and stored at 4 plus or minus 2 degrees Celsius. Five replicate spikes were determined by Method 0-4436 on days 0, 7, 14, and 28. The dashed lines at 70 and 130 percent represent the acceptable limits used to evaluate stability over time. Graphs are sorted in parameter code order. 

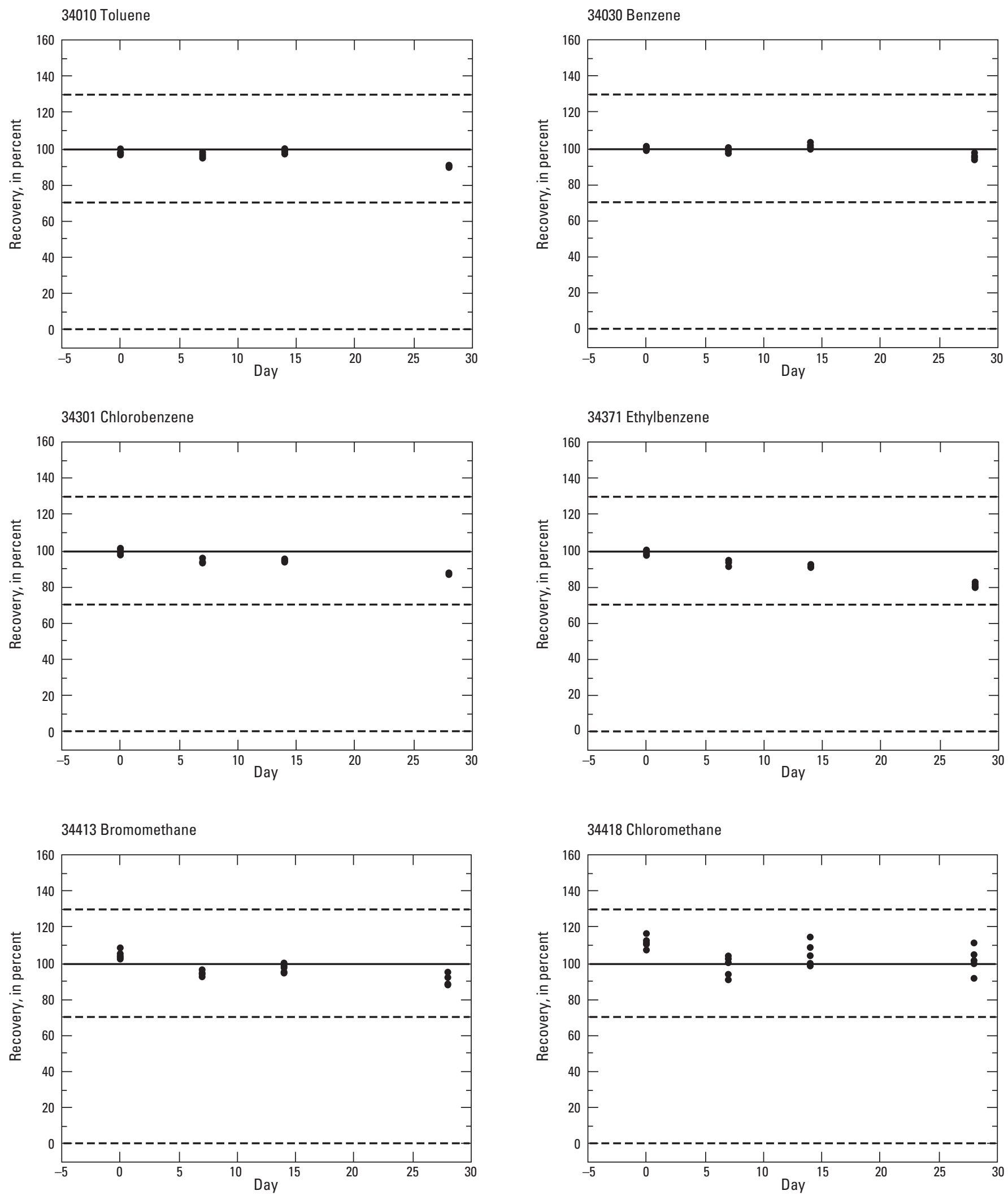

Figure 6. Summary of a 28-day holding time study for ambient purgeable volatile organic compounds. Twenty fortified replicate spikes were prepared in volatile blank water, preserved to $\mathrm{pH} 2$ with hydrochloric acid (1:1), and stored at 4 plus or minus 2 degrees Celsius. Five replicate spikes were determined by Method 0-4436 on days 0, 7, 14, and 28. The dashed lines at 70 and 130 percent represent the acceptable limits used to evaluate stability over time. Graphs are sorted in parameter code order.-Continued 

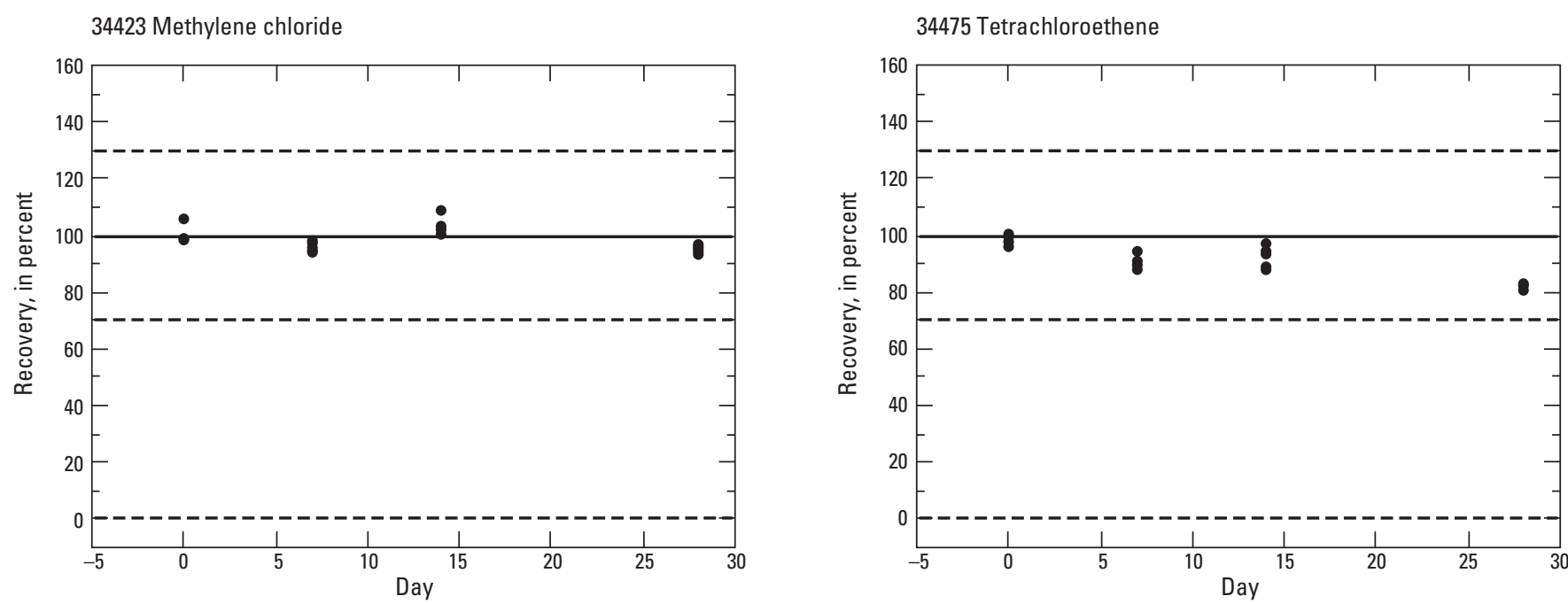

34496 1,1-Dichloroethane

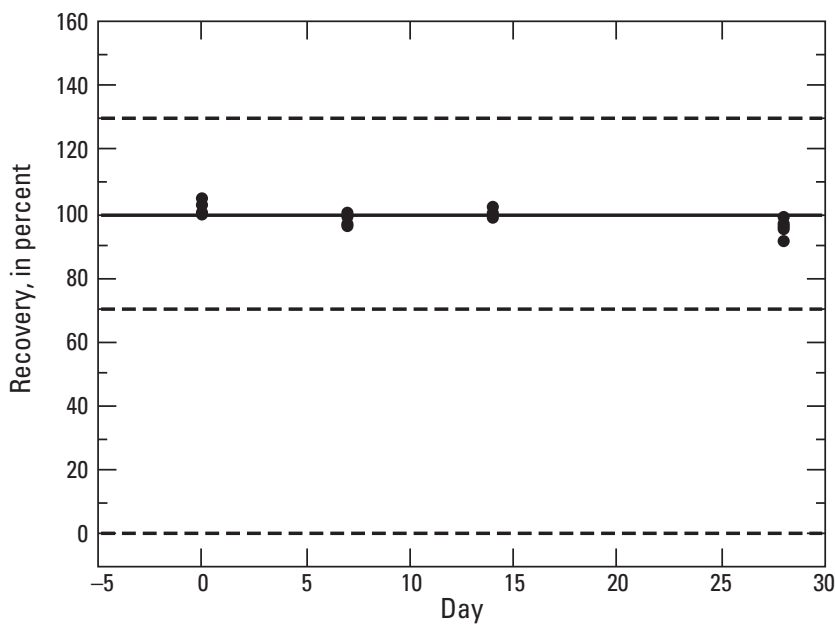

34501 1,1-Dichloroethene

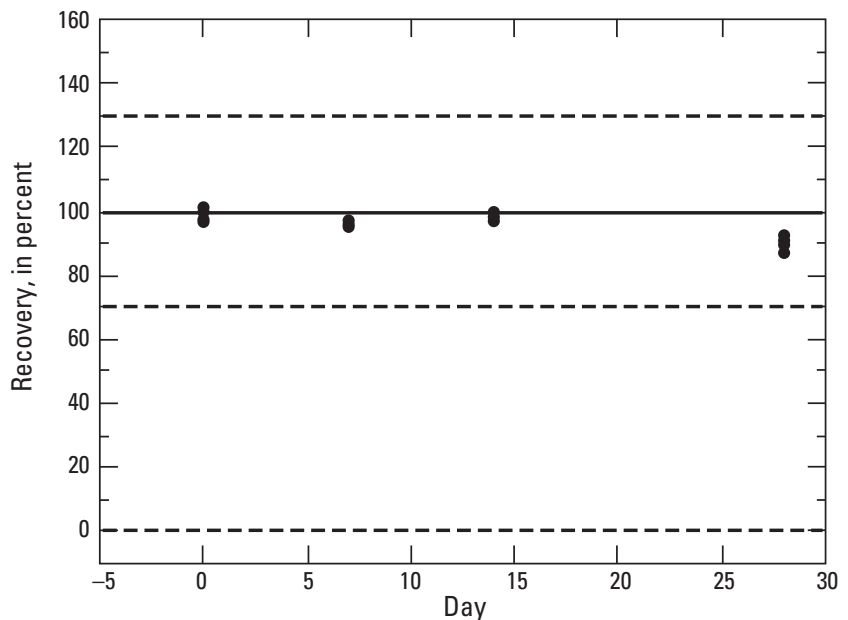

34506 1,1,1-Trichloroethane

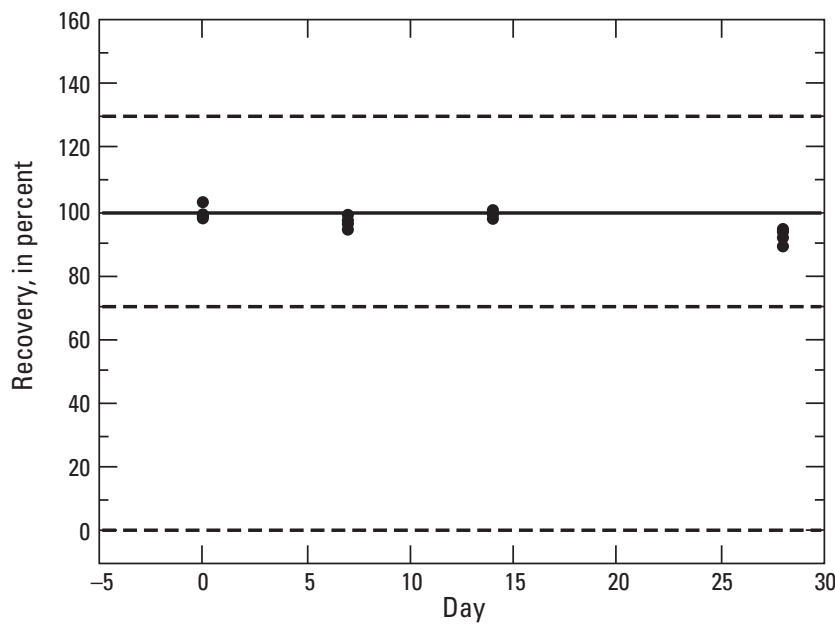

34511 1,1,2-Trichloroethane

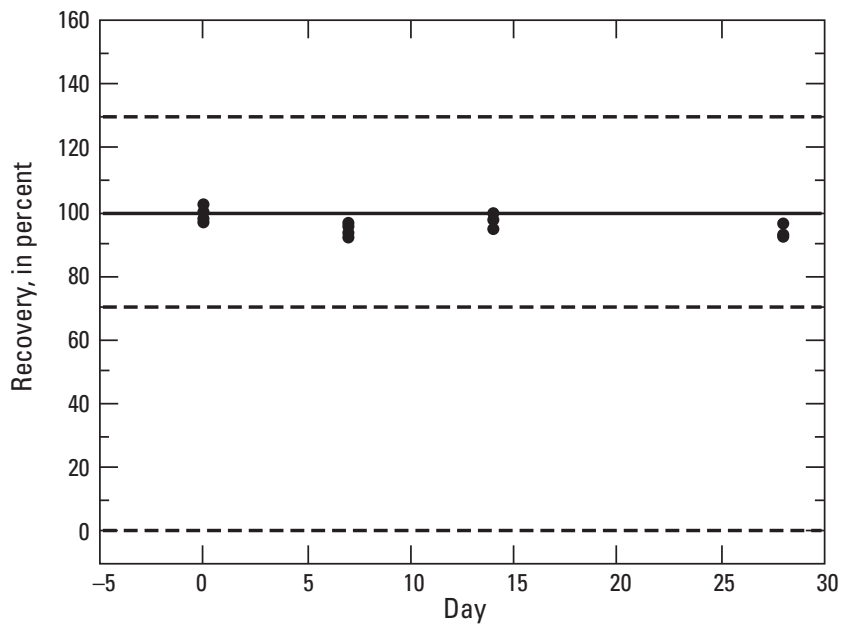

Figure 6. Summary of a 28-day holding time study for ambient purgeable volatile organic compounds. Twenty fortified replicate spikes were prepared in volatile blank water, preserved to $\mathrm{pH} 2$ with hydrochloric acid (1:1), and stored at 4 plus or minus 2 degrees Celsius. Five replicate spikes were determined by Method 0-4436 on days $0,7,14$, and 28 . The dashed lines at 70 and 130 percent represent the acceptable limits used to evaluate stability over time. Graphs are sorted in parameter code order.-Continued 
34536 1,2-Dichlorobenzene

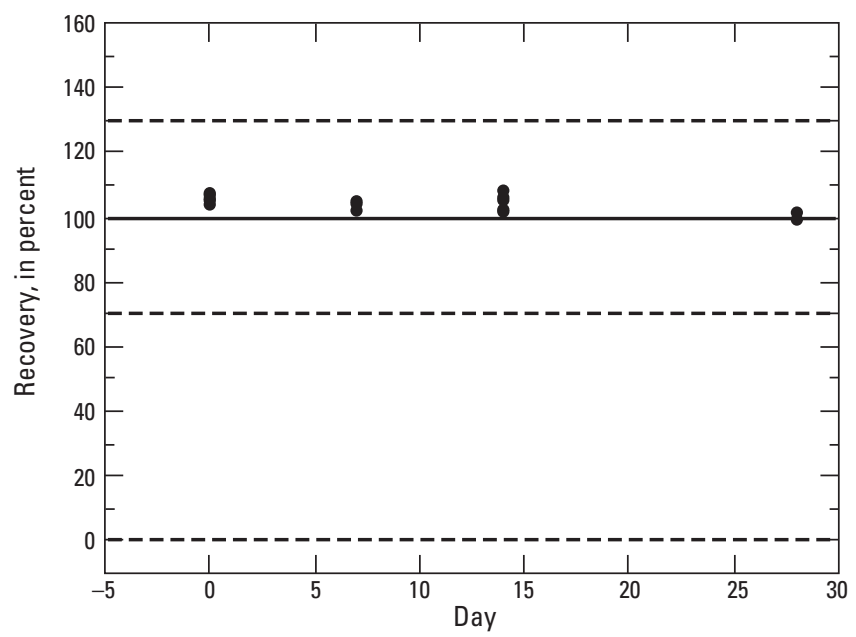

34551 1,2,4-Trichlorobenzene

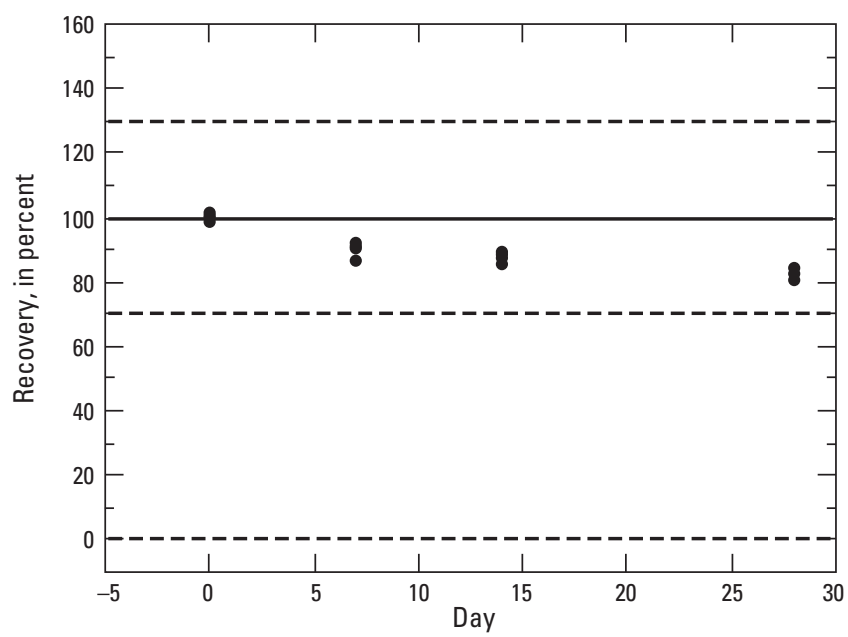

34581 2-Chloronaphthalene

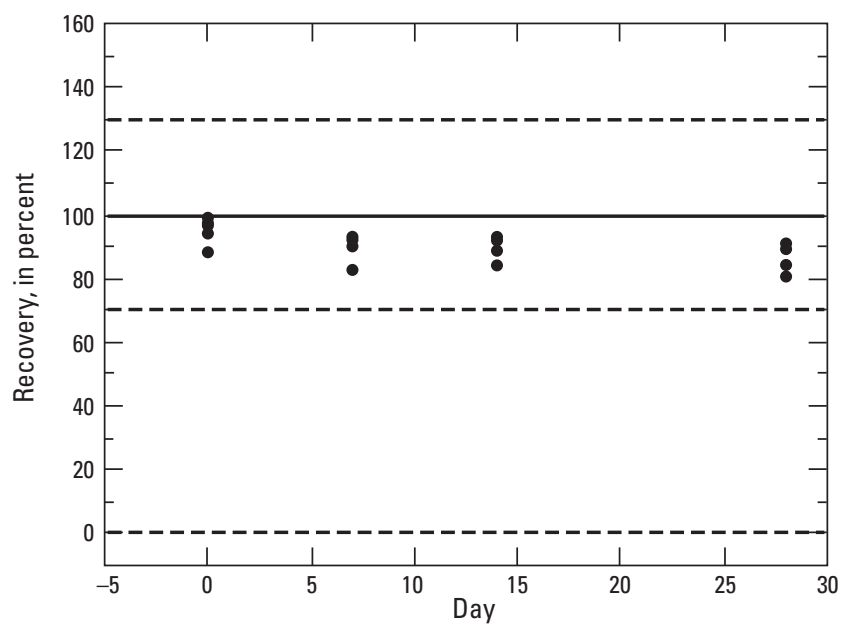

34546 trans-1,2-Dichloroethene

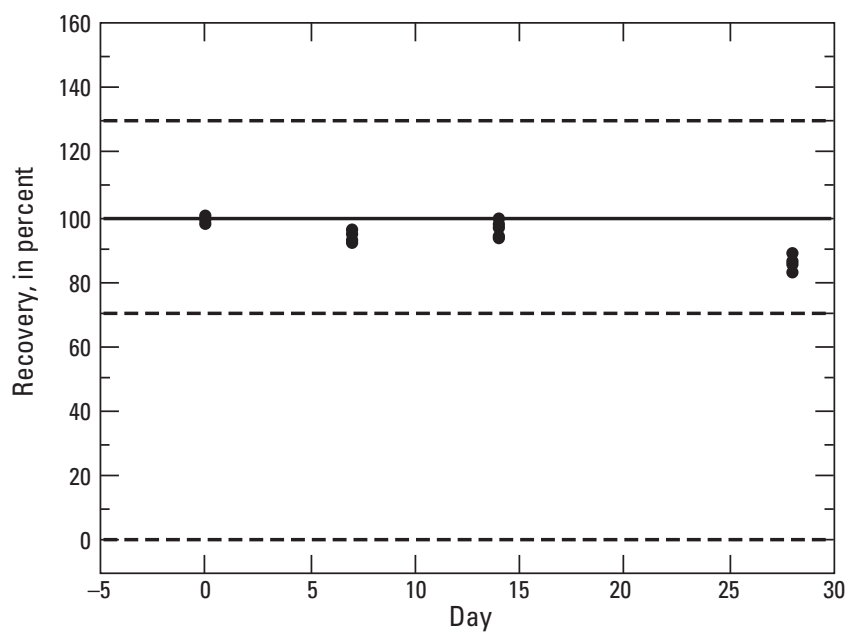

34571 1,4-Dichlorobenzene

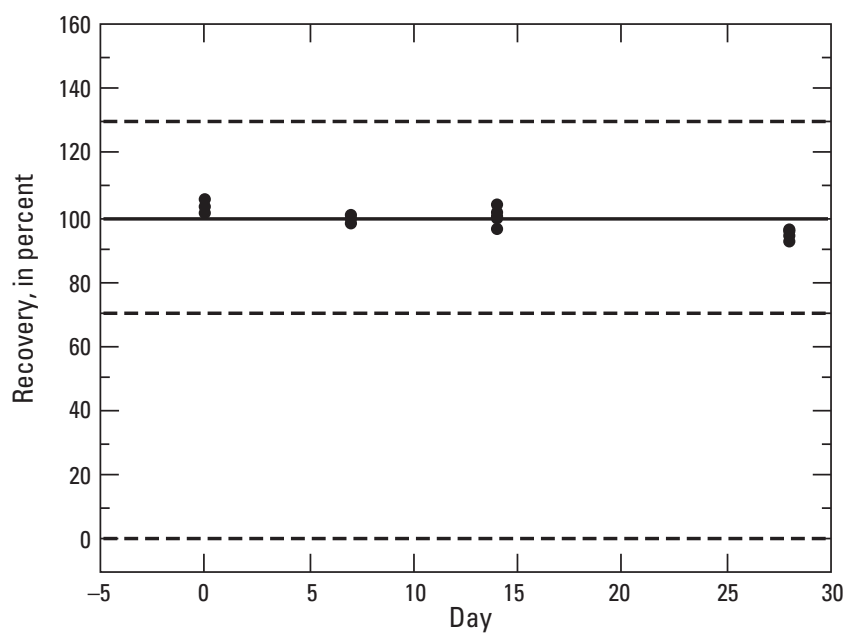

34696 Naphthalene

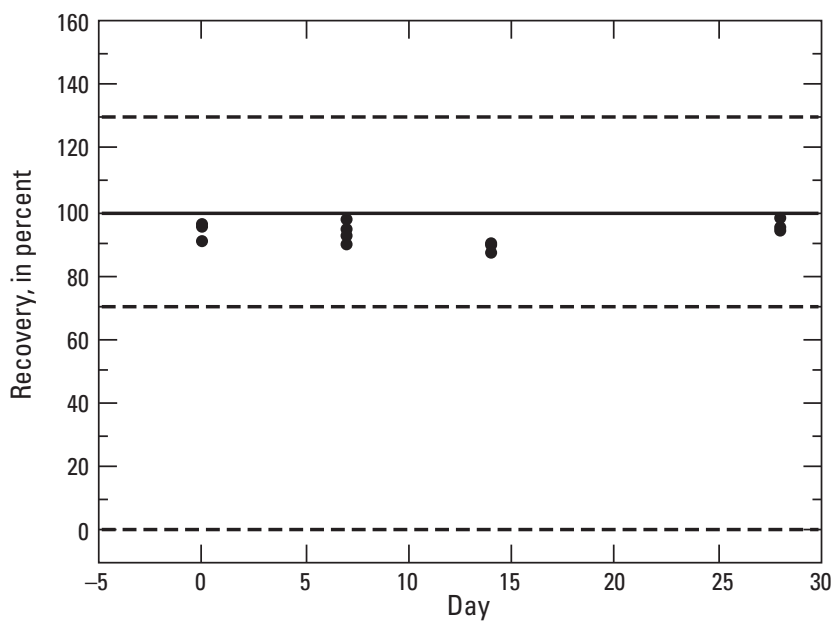

Figure 6. Summary of a 28-day holding time study for ambient purgeable volatile organic compounds. Twenty fortified replicate spikes were prepared in volatile blank water, preserved to $\mathrm{pH} 2$ with hydrochloric acid (1:1), and stored at 4 plus or minus 2 degrees Celsius. Five replicate spikes were determined by Method 0-4436 on days 0, 7, 14, and 28. The dashed lines at 70 and 130 percent represent the acceptable limits used to evaluate stability over time. Graphs are sorted in parameter code order.-Continued 
34699 trans-1,3-Dichloropropene

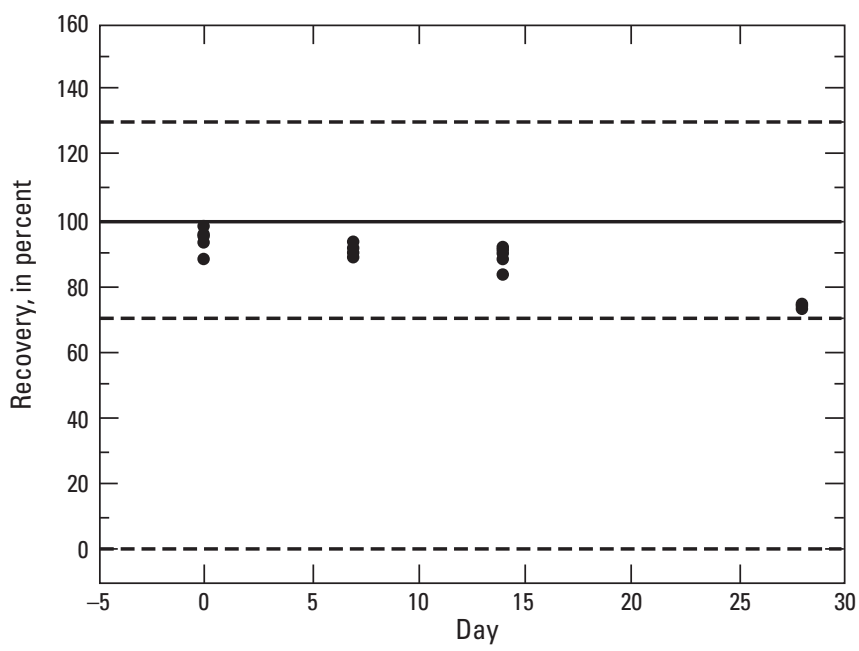

39175 Vinyl chloride

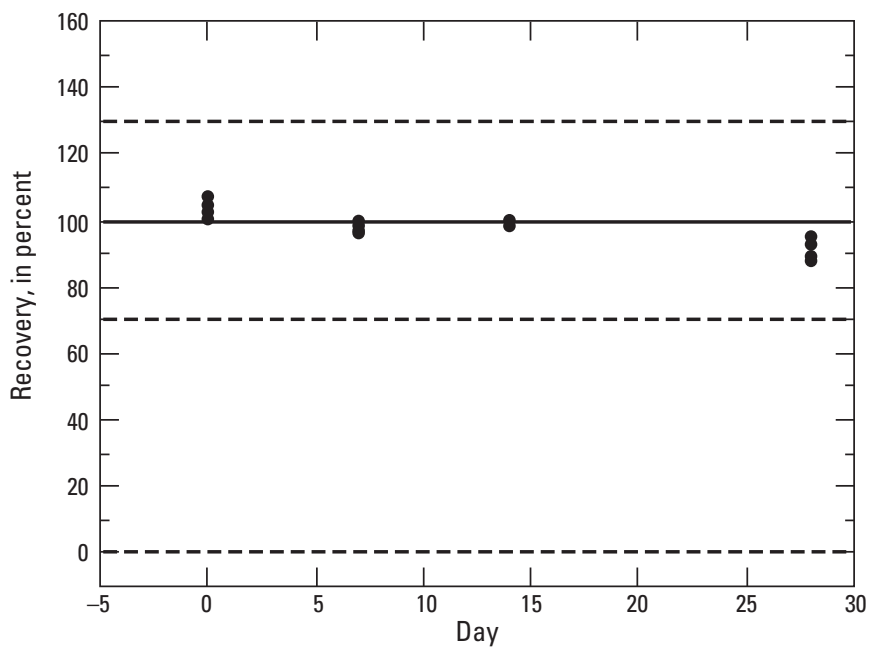

45028 Chlorodifluoromethane

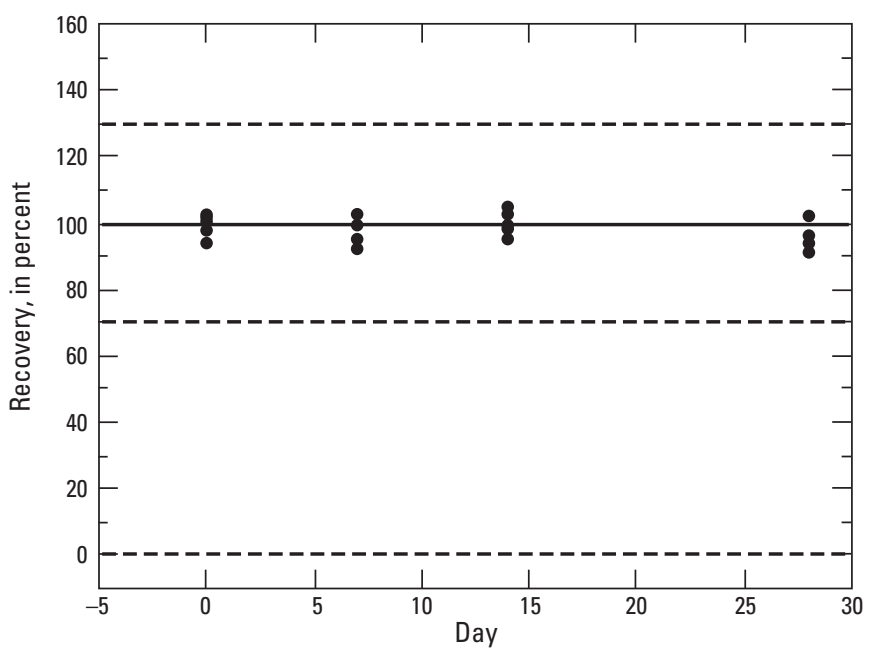

34704 cis-1,3-Dichloropropene

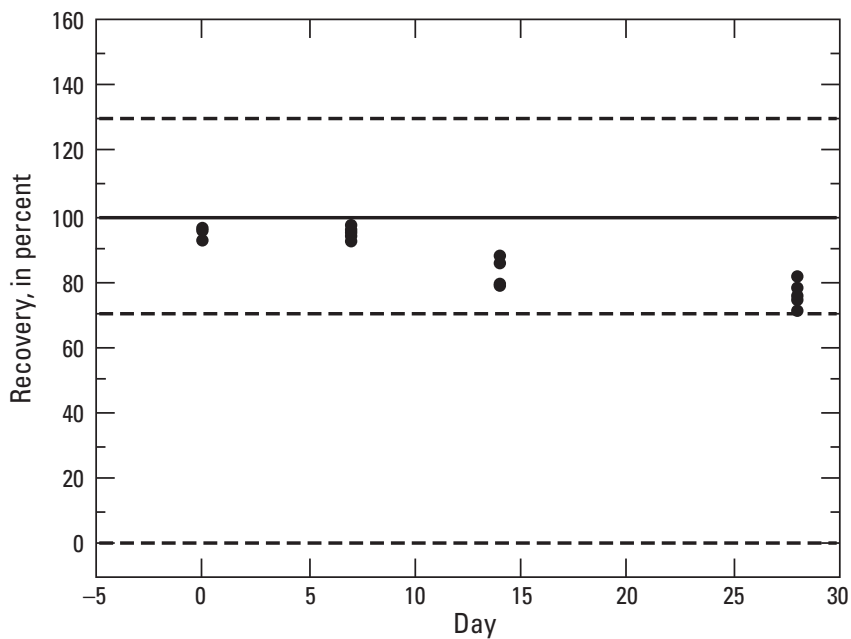

39180 Trichloroethene

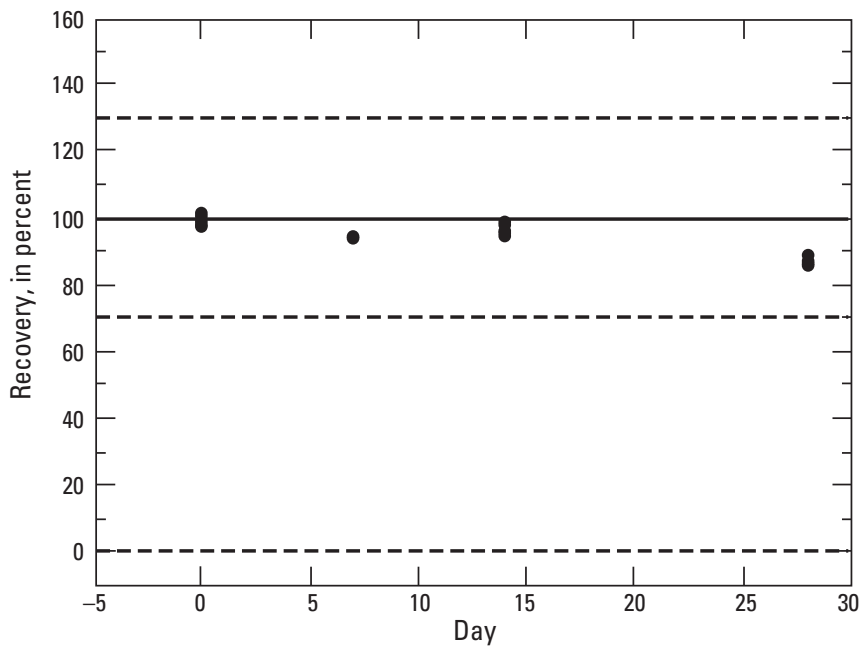

49538 1,1-Difluoroethane

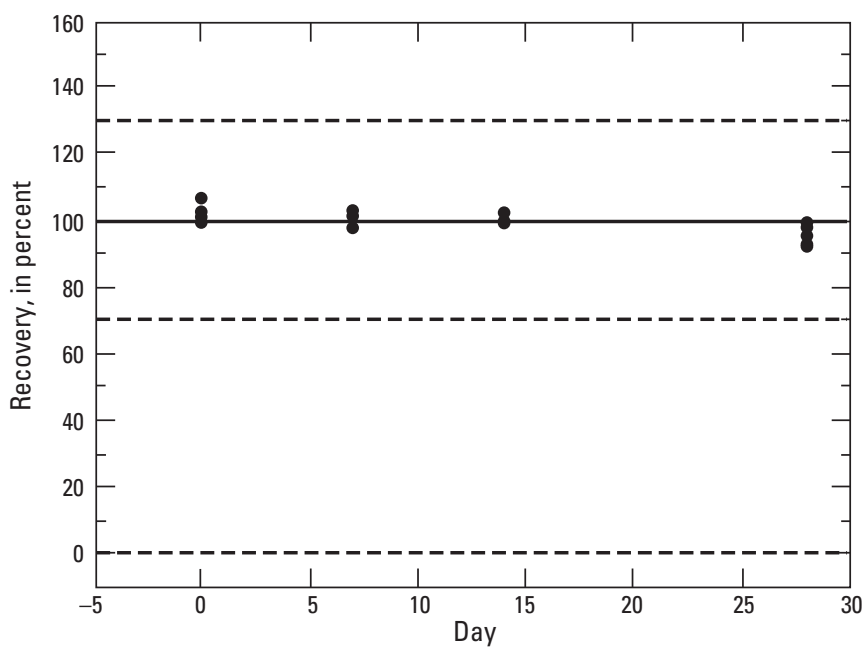

Figure 6. Summary of a 28-day holding time study for ambient purgeable volatile organic compounds. Twenty fortified replicate spikes were prepared in volatile blank water, preserved to $\mathrm{pH} 2$ with hydrochloric acid (1:1), and stored at 4 plus or minus 2 degrees Celsius. Five replicate spikes were determined by Method 0-4436 on days $0,7,14$, and 28 . The dashed lines at 70 and 130 percent represent the acceptable limits used to evaluate stability over time. Graphs are sorted in parameter code order.-Continued 
50985 1,2-Dichloro-1,1,2,2-tetrafluoroethane

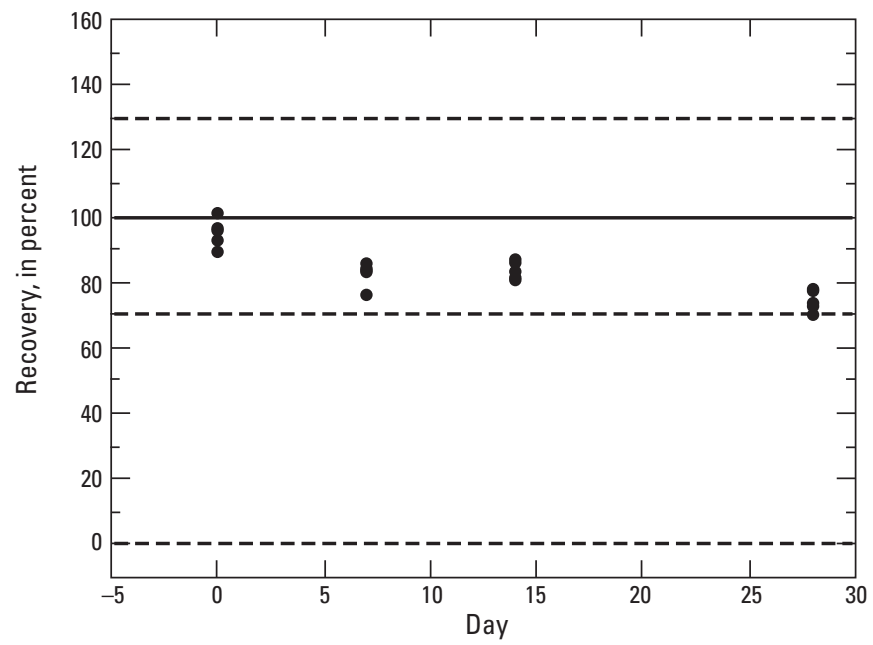

62174 2,2-Dichloro-1,1,1-trifluoroethane

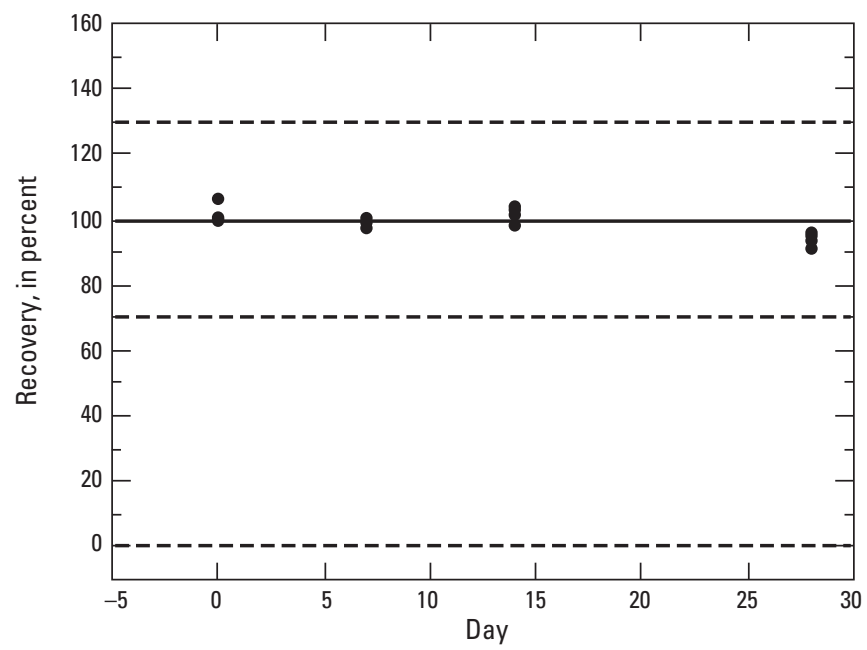

68726 1,3-Butadiene

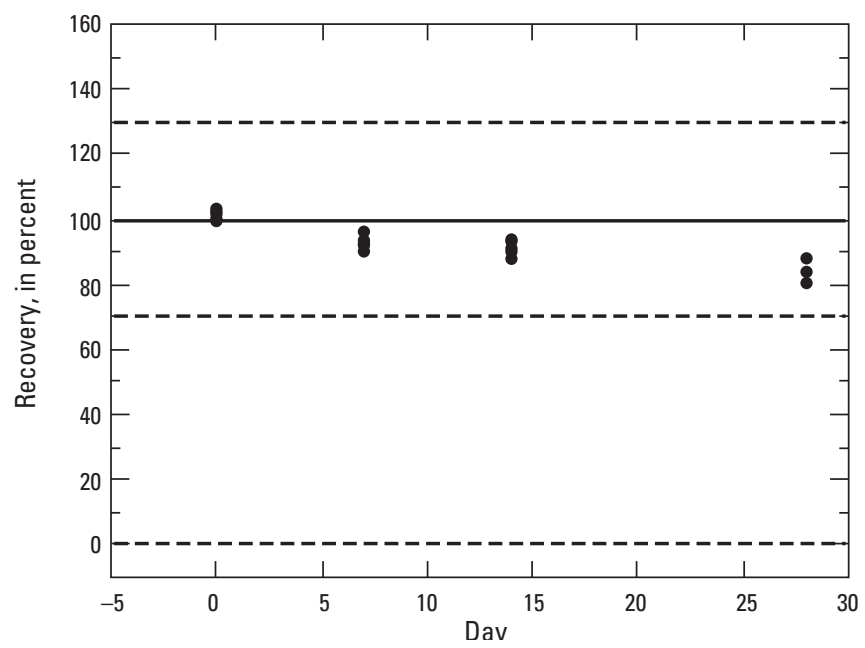

61701 2,6-Di-tert-butyl phenol

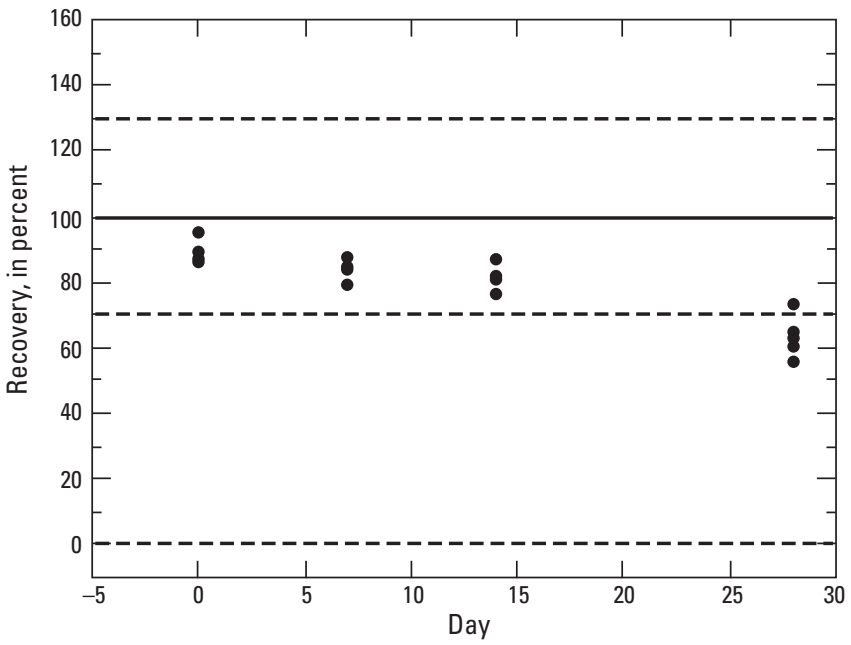

68725 1,2-Dimethylnaphthalene

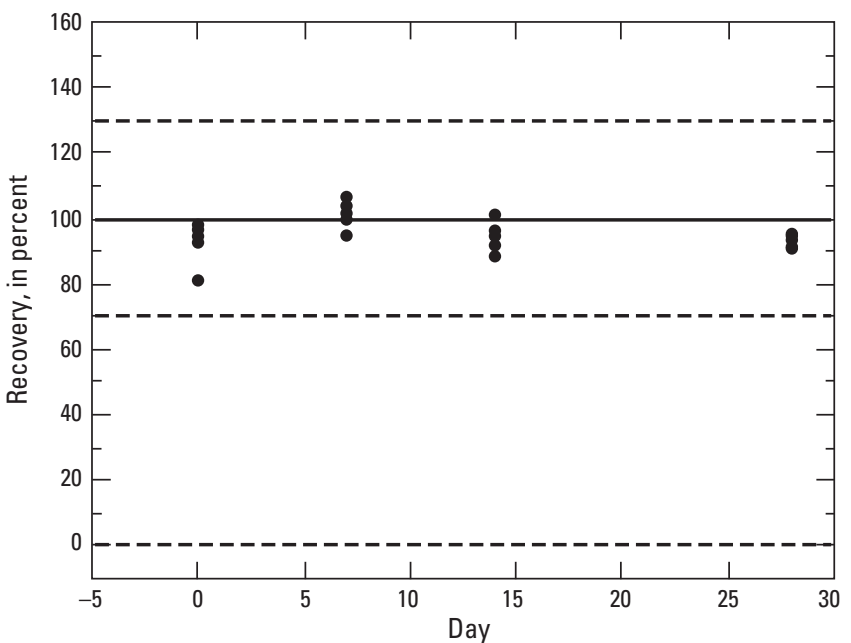

68727 1,6-Dimethylnaphthalene

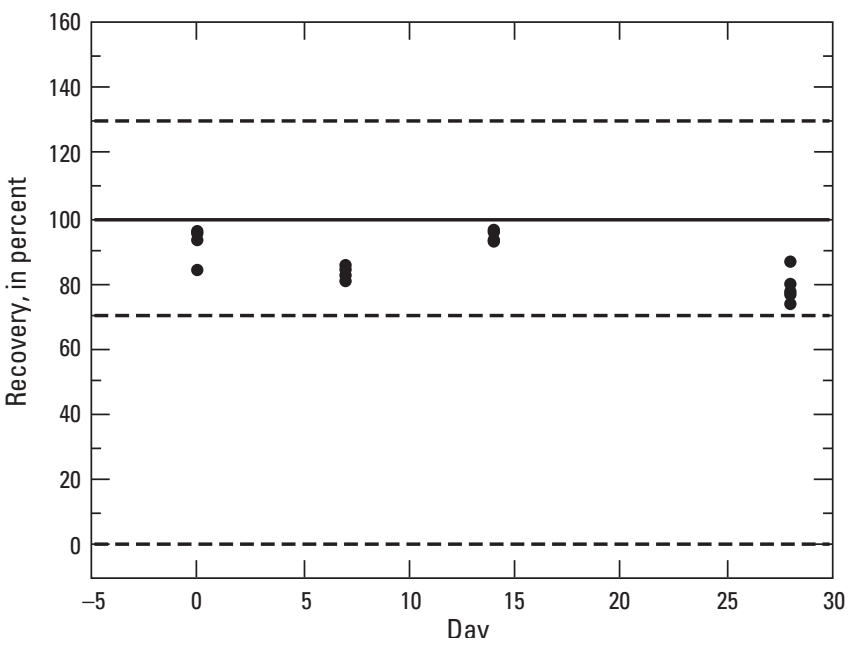

Figure 6. Summary of a 28-day holding time study for ambient purgeable volatile organic compounds. Twenty fortified replicate spikes were prepared in volatile blank water, preserved to $\mathrm{pH} 2$ with hydrochloric acid (1:1), and stored at 4 plus or minus 2 degrees Celsius. Five replicate spikes were determined by Method 0-4436 on days $0,7,14$, and 28 . The dashed lines at 70 and 130 percent represent the acceptable limits used to evaluate stability over time. Graphs are sorted in parameter code order.-Continued 
68731 Benzyl chloride

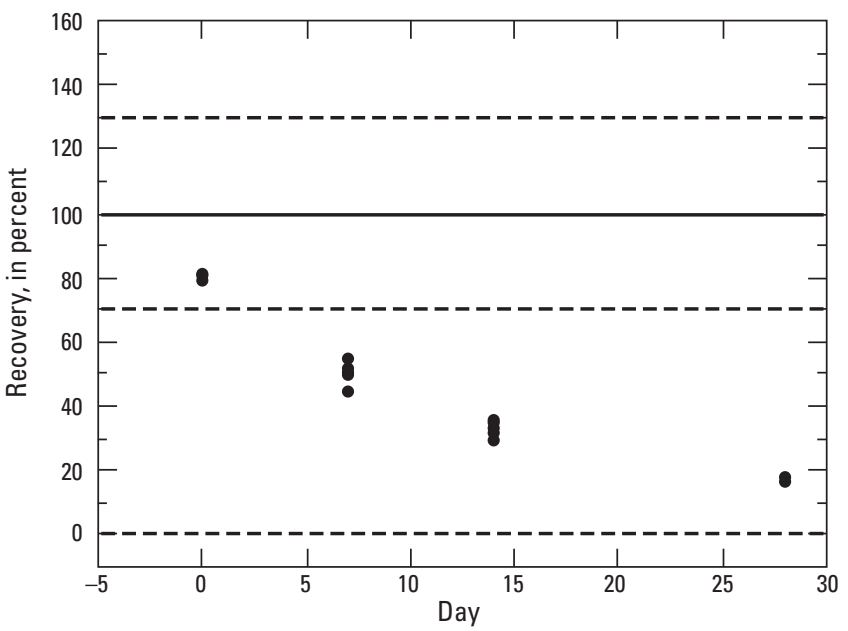

77093 cis-1,2-Dichloroethene

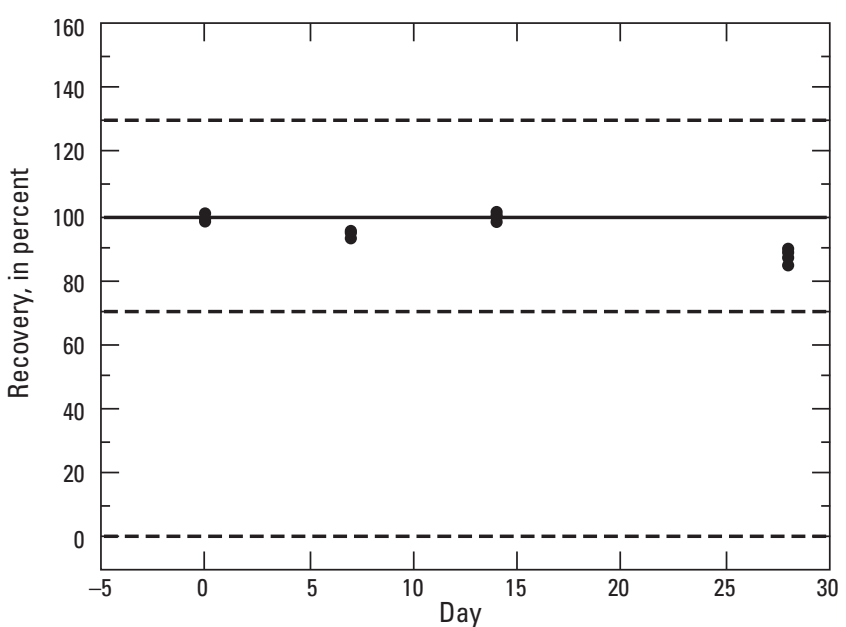

77128 Styrene

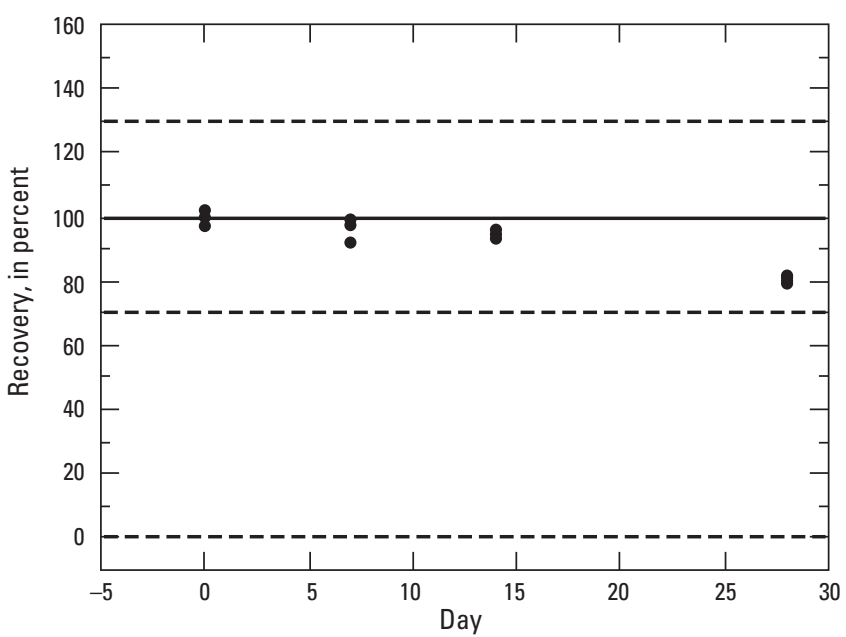

77041 Carbon disulfide

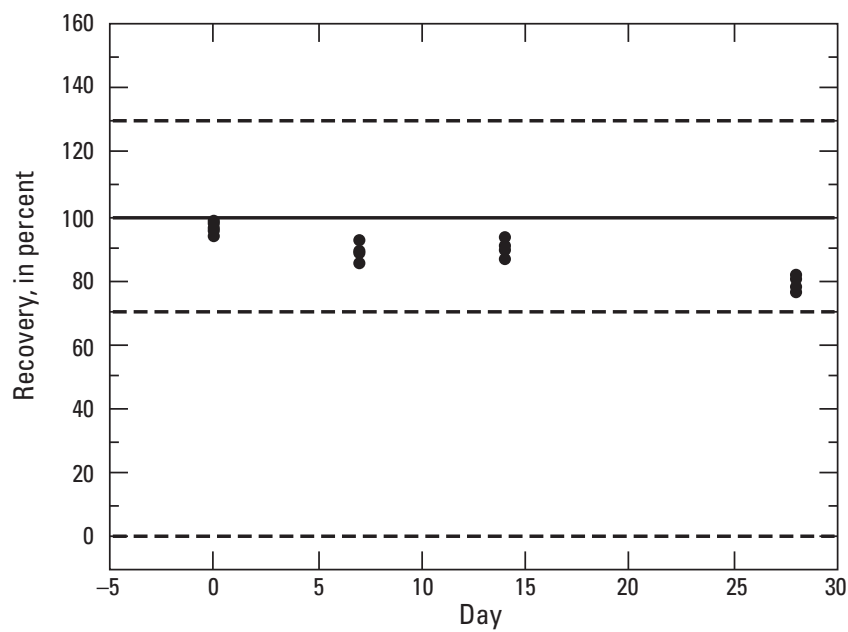

77119 Dichlorofluoromethane

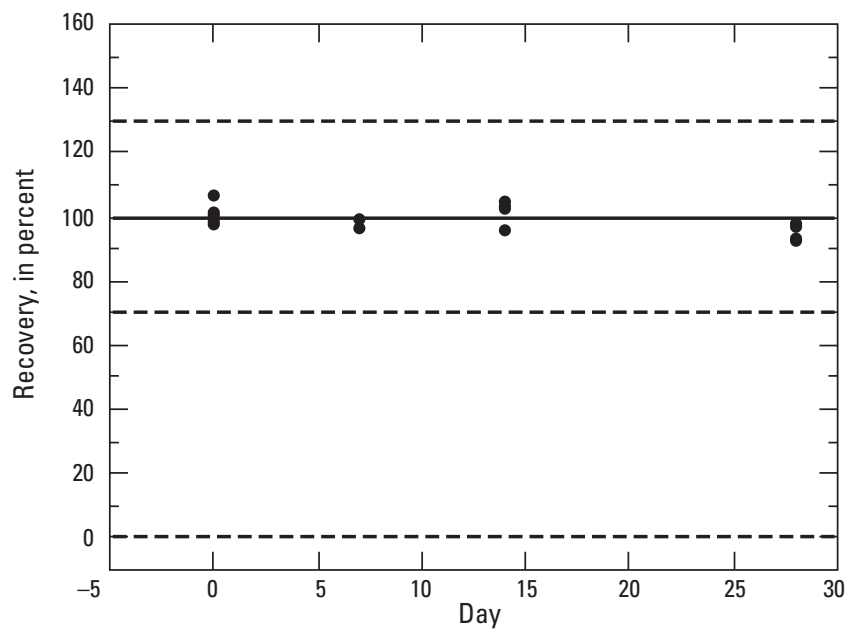

77135 ortho-Xylene

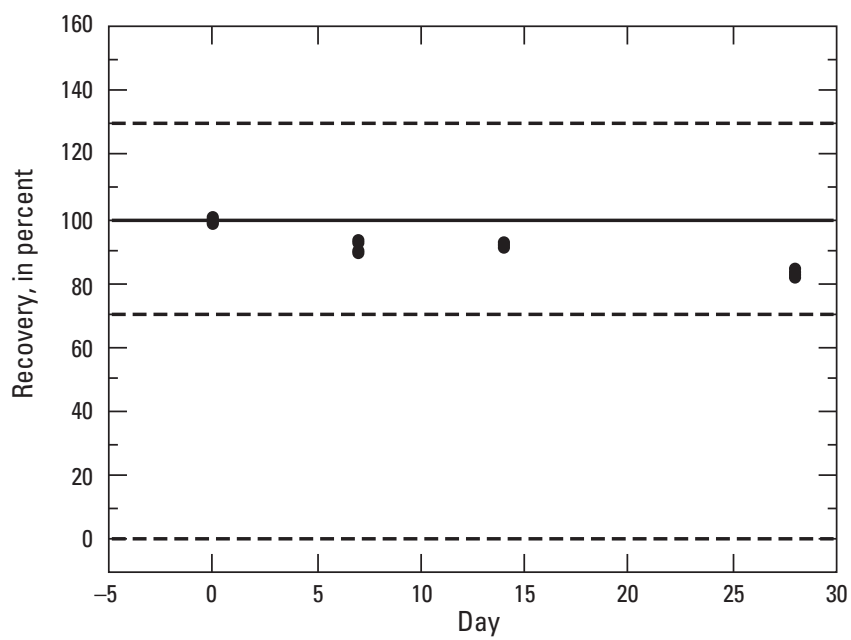

Figure 6. Summary of a 28-day holding time study for ambient purgeable volatile organic compounds. Twenty fortified replicate spikes were prepared in volatile blank water, preserved to $\mathrm{pH} 2$ with hydrochloric acid (1:1), and stored at 4 plus or minus 2 degrees Celsius. Five replicate spikes were determined by Method 0-4436 on days 0, 7, 14, and 28. The dashed lines at 70 and 130 percent represent the acceptable limits used to evaluate stability over time. Graphs are sorted in parameter code order.-Continued 
77222 1,2,4-Trimethylbenzene

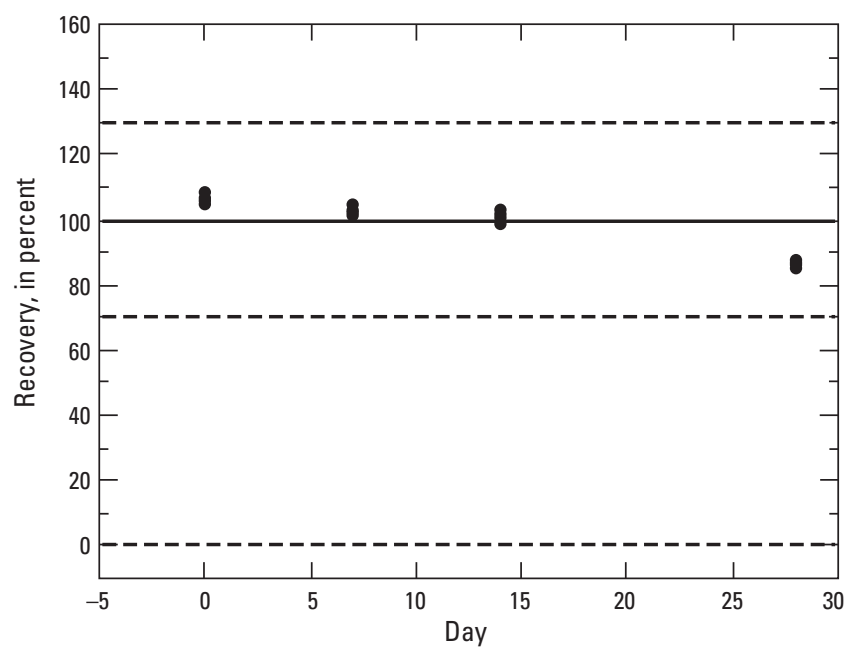

77297 Bromochloromethane

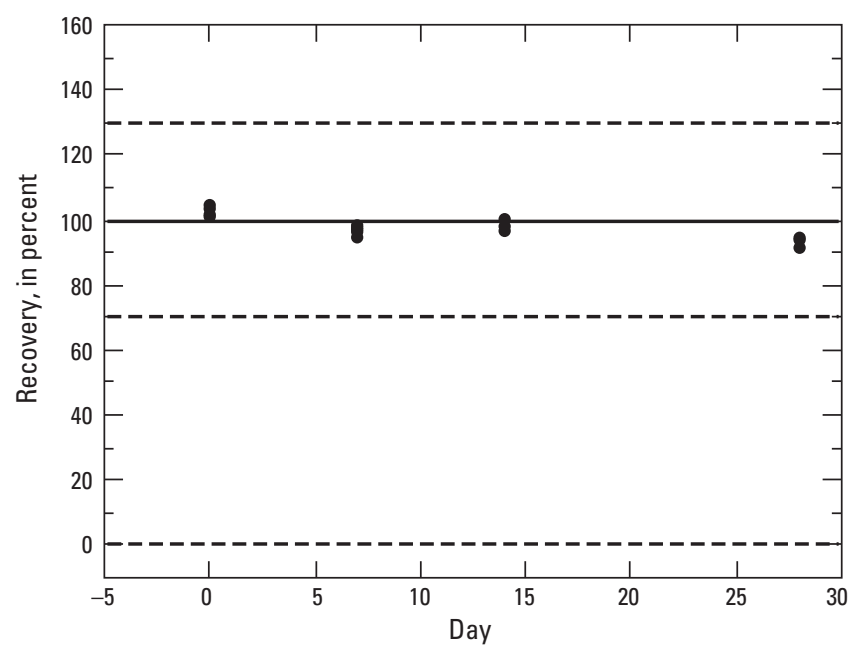

77350 sec-Butylbenzene

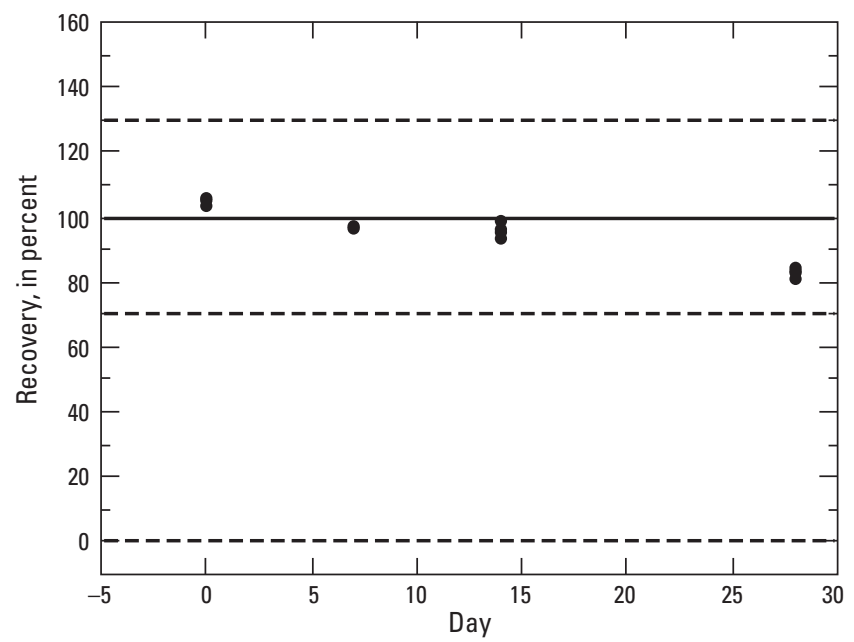

77224 n-Propylbenzene

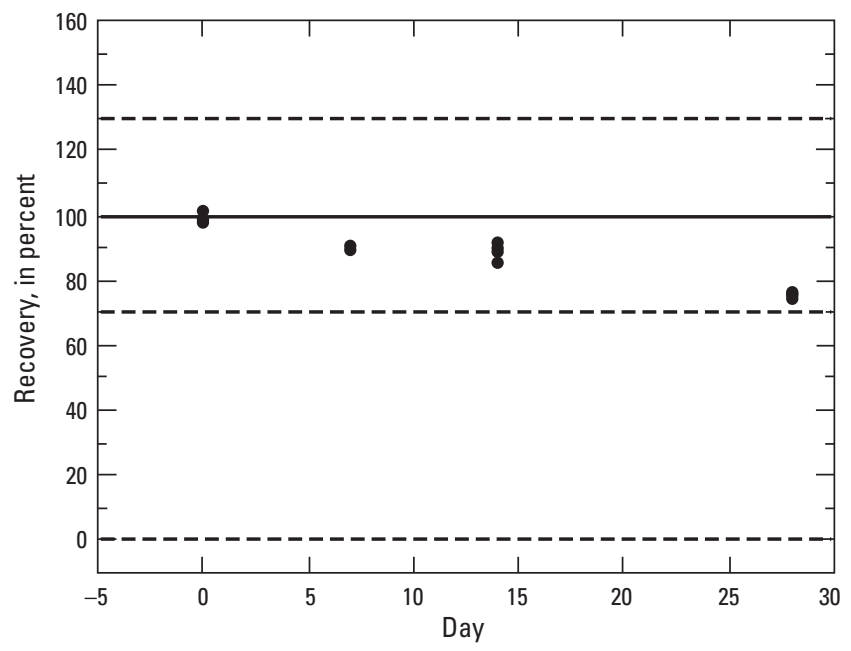

77323 1,2,3,4-Tetrahydronaphthalene

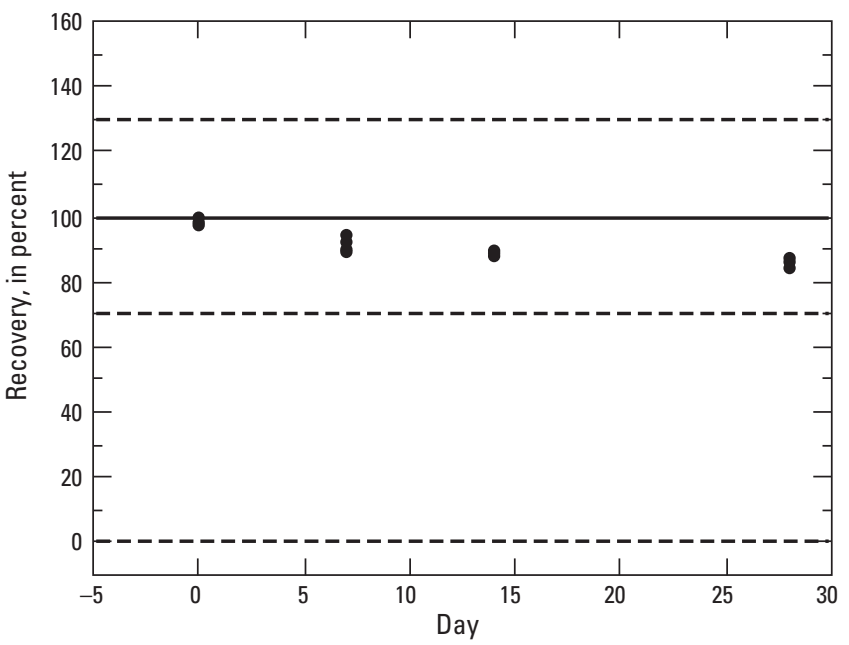

77562 1,1,1,2-Tetrachloroethane

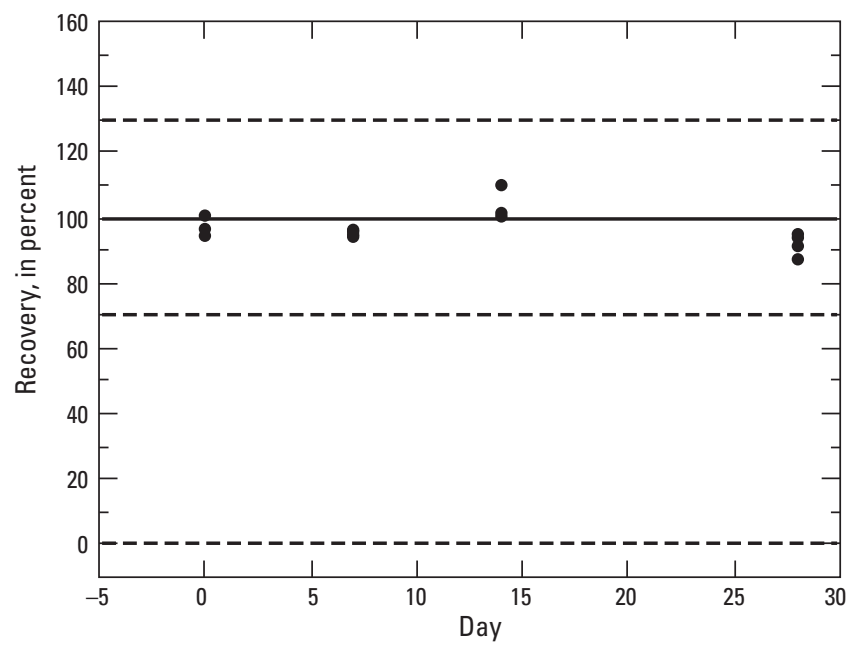

Figure 6. Summary of a 28-day holding time study for ambient purgeable volatile organic compounds. Twenty fortified replicate spikes were prepared in volatile blank water, preserved to $\mathrm{pH} 2$ with hydrochloric acid (1:1), and stored at 4 plus or minus 2 degrees Celsius. Five replicate spikes were determined by Method 0-4436 on days 0, 7, 14, and 28. The dashed lines at 70 and 130 percent represent the acceptable limits used to evaluate stability over time. Graphs are sorted in parameter code order.-Continued 
78032 tert-Butyl methyl ether (MTBE)

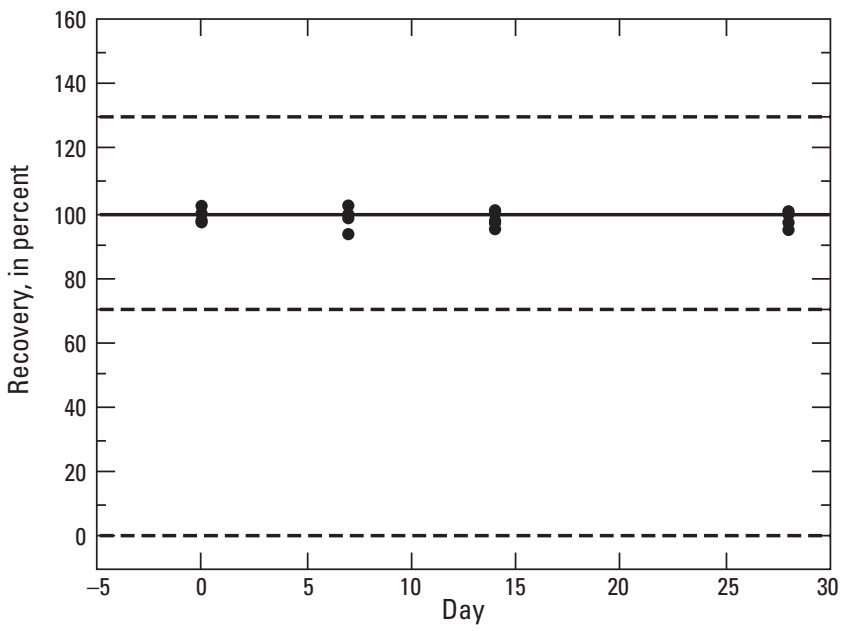

81590 Hexane

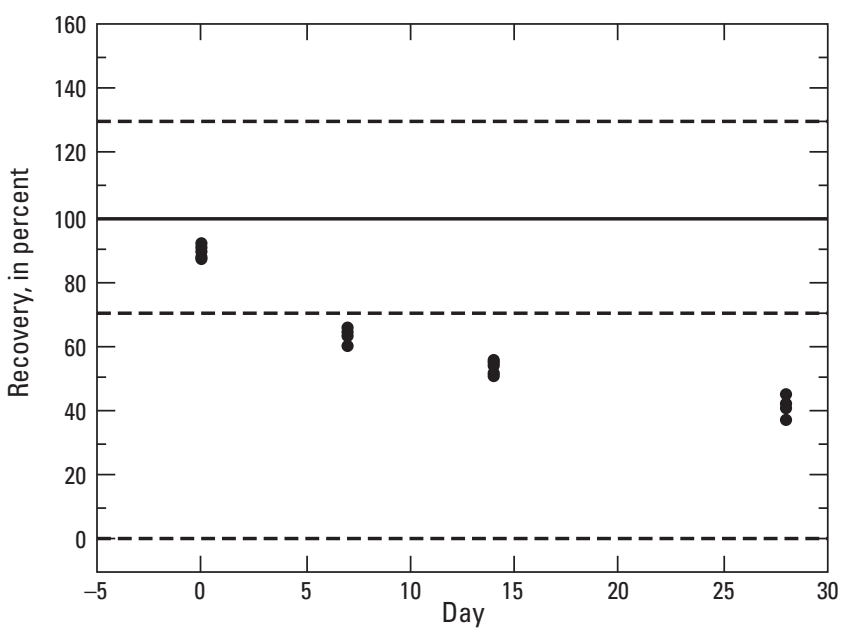

85668 1-Chloro-1,1-difluoroethane

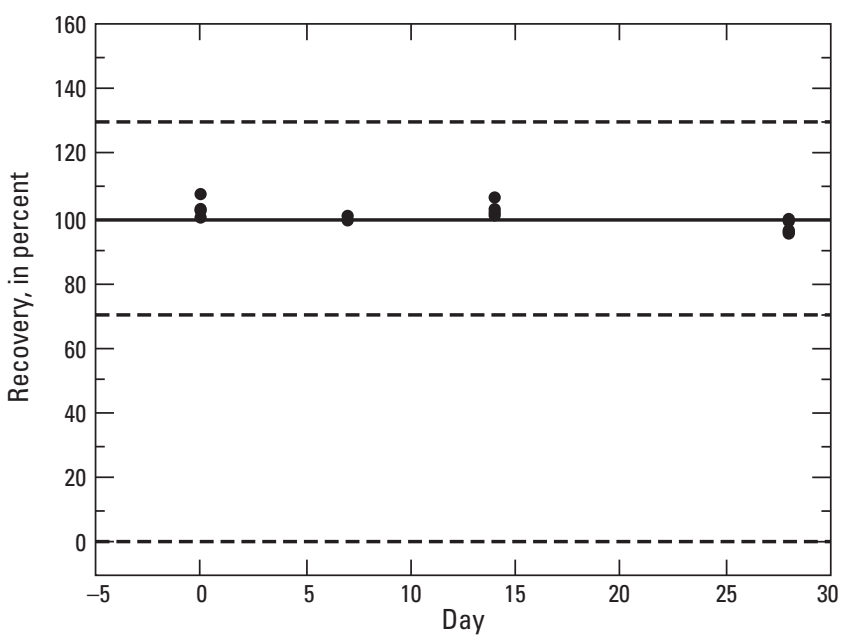

81563 Butane

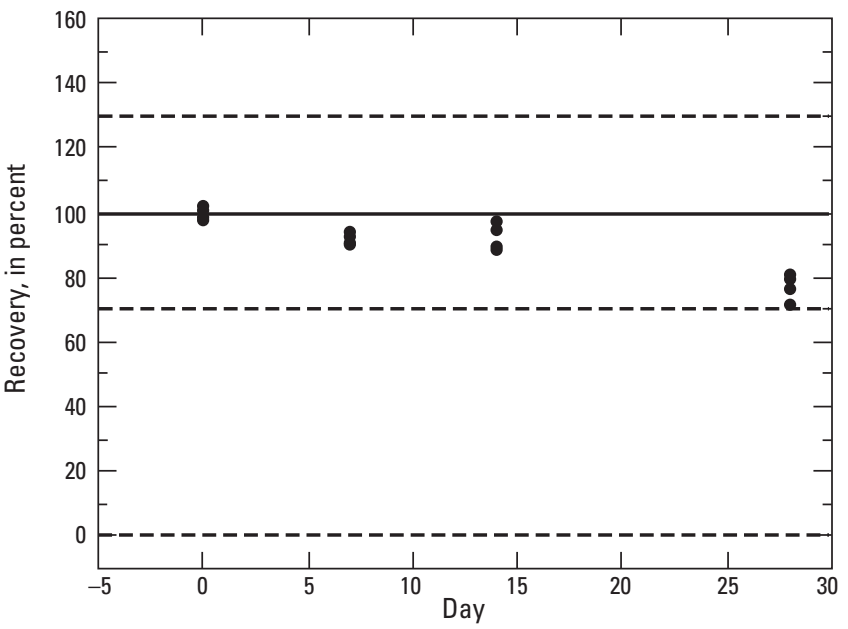

81604 Pentane

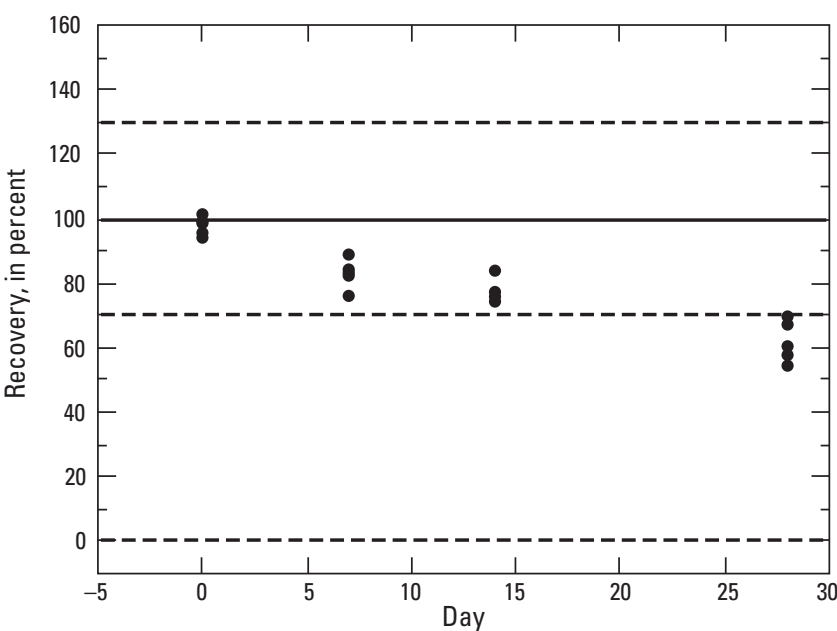

85795 meta- and para-Xylene

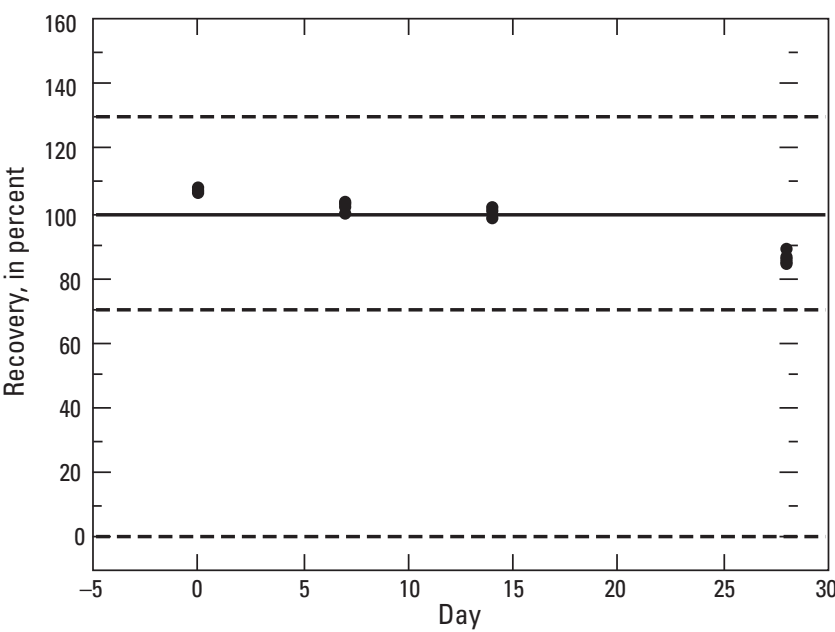

Figure 6. Summary of a 28-day holding time study for ambient purgeable volatile organic compounds. Twenty fortified replicate spikes were prepared in volatile blank water, preserved to $\mathrm{pH} 2$ with hydrochloric acid (1:1), and stored at 4 plus or minus 2 degrees Celsius. Five replicate spikes were determined by Method 0-4436 on days 0, 7, 14, and 28. The dashed lines at 70 and 130 percent represent the acceptable limits used to evaluate stability over time. Graphs are sorted in parameter code order.-Continued 
The majority of ambient purgeable VOCs have calculated holding times using the ASTM procedure of greater than 14 days. Among the ambient purgeable VOCs, only 6 compounds have calculated holding times less than 14 days (table 18). Calculated ASTM holding times were 8 days for 1,2-dichloro1,1,2,2-tetrafluoroethane, 12 days for 1,3-butadiene, 10 days for butane, 8 days for pentane, and 12 days for cis-1,3-dichloropropene. On day 14 mean recoveries for 1,2-dichloro-1,1,2,2-tetrafluoroethane (84 percent), 1,3-butadiene (91 percent), butane (92 percent), pentane (77 percent), and cis-1,3-dichloropropene (83 percent) were within data quality objectives (70-130 percent) (table 17). These compounds will be assigned a 14-day holding time based upon the recoveries on day 14. The calculated ASTM holding time for hexane was 6 days; mean recovery on day 14 was 53 percent. Consequently, hexane will be reported with an "E" remark code to indicate an estimated concentration because the performance falls outside the acceptable range (70-130 percent). However, hexane will be retained in Method O-4436 until more data are available and evaluated. It may be eliminated from the method upon further review. The calculated ASTM holding time for benzyl chloride was 6 days; mean recovery on day 14 was 33 percent. Benzyl chloride was eliminated from the method due to instability.

Preservation with $\mathrm{HCl}(1: 1)$ to $\mathrm{pH} 2$ is recommended for all ambient purgeable VOCs in Method O-4436. A 14-day holding time is supported for most compounds by the ASTM holding time calculations and for all compounds based upon the recovery data generated for day 14 of the holding time study, excluding hexane and benzyl chloride.

\subsection{Results and Discussion of Laboratory and Environmental Samples Determined by Method 0-4437}

12.4.1. Laboratory Continuing Calibration Verification (CCV) Standards-Seventy-five heat purgeable $\mathrm{CCVs}$ were determined from January through August 2013 using Method O-4437. Mean recoveries ranged from 84 to 111 percent, with RSDs $\leq 19$ percent. Compounds with RSDs greater than 10 percent include 1-methoxy-4-(2-propenyl) benzene, alpha-terpineol, hexachlorocyclopentadiene, isophorone, and N-nitrosodiethylamine (table 19). The performance for acrolein was within data quality objectives, with a mean recovery 87 percent, ranging from 69 to 100 percent, and an RSD of 7 percent. However, difficulties were encountered trying to obtain standard solutions that were in agreement with each other. Acrolein was eliminated from the method after further review of data collected from 2013 through 2015.

12.4.2. Laboratory Reagent-Water Blanks (LRB)_Eightyfour LRBs were determined from January through August 2013 using Method O-4437. Eight compounds were detected in the LRBs. 2-Ethyl-1-hexanol had a 100 percent detection frequency in the LRBs. The 99th percentile concentration was $2.1 \mu \mathrm{g} / \mathrm{L}$. 2-Ethyl1-hexanol can be emitted from carpets and some plastics, and is a major metabolite of di-(2-ethylhexyl) phthalate (New Jersey Department of Environmental Protection, 2008). 2-Ethyl-1-hexanol is reported as a blank-limited compound with a MRL of $<2 \mu \mathrm{g} / \mathrm{L}$. 1-Octanol was detected in 69 percent of LRBs at concentrations less than the MDL of $0.9 \mu \mathrm{g} / \mathrm{L}$. The 99th percentile concentration was $0.22 \mu \mathrm{g} / \mathrm{L}$. Isopropyl alcohol was detected in 15 percent of the LRBs. Two detections exceeded the MDL of $0.4 \mu \mathrm{g} / \mathrm{L}$. The 99 th percentile concentration was $0.53 \mu \mathrm{g} / \mathrm{L}$. Hexachlorocyclopentadiene was detected in 12 percent of the LRBs at concentrations less than the MDL of $0.8 \mu \mathrm{g} / \mathrm{L}$. The 99 th percentile concentration was $0.08 \mu \mathrm{g} / \mathrm{L}$. All detections for 1-octanol and hexachlorocyclopentadiene resulted from residual carryover in the instrument after analyzing the higher concentration calibration standards. Cyclohexanone, 1-methoxy-4-(2-propenyl) benzene, acetonitrile, and alpha-terpineol were detected in 5 percent or less of the LRBs. Table 20 summarizes results of LRBs.

In the early method development stages, tert-butyl alcohol was observed to be a contaminant from the $\mathrm{HCl}(1: 1)$ preservative if the stock hydrochloric acid were purchased in a high density polyethylene bottle. Preservative prepared from hydrochloric acid stocks purchased in glass, Teflon ${ }^{\circledR}$, or perfluoroalkyl fluoropolymer bottles were free of tert-butyl alcohol at the RL when six drops of $\mathrm{HCl}(1: 1)$ were added to a 40-mL VOC vial containing VBW.

12.4.3. Field-Submitted Blanks (FSB)_Eighty-six FSBs, collected from diverse groundwater and surface-water sites across the United States, were analyzed from January to August 2013. FSBs included field blanks, equipment blanks, source solution blanks, and trip blanks. The most commonly detected compounds in FSBs, except for the blank-limited 2-ethyl-1-hexanol, were acetonitrile, ethyl acetate, isopropyl alcohol, and tert-butyl alcohol (table 21). Acetonitrile was the most frequently detected compound, found in 29 percent of the FSBs with a maximum detected concentration of $0.5 \mu \mathrm{g} / \mathrm{L}(\mathrm{RL}<0.8 \mu \mathrm{g} / \mathrm{L})$. Only two concentrations exceeded the MDL of $0.4 \mu \mathrm{g} / \mathrm{L}$. Ethyl acetate was detected in 10 percent of the 
FSBs, with a maximum detected concentration of $0.11 \mu \mathrm{g} / \mathrm{L}$. All concentrations were greater than the MDL of $0.03 \mu \mathrm{g} / \mathrm{L}$. Isopropyl alcohol and tert-butyl alcohol were detected in 9 percent of the FSBs. The maximum concentration for isopropyl alcohol was $7.5 \mu \mathrm{g} / \mathrm{L}$, with all values greater than the MDL of $0.4 \mu \mathrm{g} / \mathrm{L}$. The maximum concentration for tertbutyl alcohol was $1.36 \mu \mathrm{g} / \mathrm{L}$, with seven of the values exceeding the MDL of $0.12 \mu \mathrm{g} / \mathrm{L}$. 1-Octanol, MTBE, 1-butanol, and cyclohexanone were detected in less than or equal to 3 percent of the FSBs.

Acetonitrile was detected in 4 percent of the LRBs (table 20) at concentrations ranging from 0.03 to $0.09 \mu \mathrm{g} / \mathrm{L}$. Isopropyl alcohol was detected in 15 percent of the LRBs, ranging in concentration from 0.1 to $0.5 \mu \mathrm{g} / \mathrm{L}$. Isopropyl alcohol concentrations ranging from 0.2 to $0.4 \mu \mathrm{g} / \mathrm{L}$ were reported as $<\mathrm{RL}(<0.8 \mu \mathrm{g} / \mathrm{L})$ in five FSBs in this dataset because of the presence of isopropyl alcohol in the LRB. 1-Octanol concentrations were reported as $<\mathrm{RL}(<1.8 \mu \mathrm{g} / \mathrm{L})$ for three FSBs because of the presence of 1-octanol in the LRB.

2-Ethyl-1-hexanol is a blank-limited compound with a MRL of $<2 \mu \mathrm{g} / \mathrm{L}$. 2-Ethyl-1-hexanol was detected in 85 of the $86 \mathrm{FSBs}$, at concentrations ranging from 0.1 to $1.5 \mu \mathrm{g} / \mathrm{L}$. The median concentration was $0.6 \mu \mathrm{g} / \mathrm{L}$. The sample results were reported as $<\mathrm{MRL}$ $(<2 \mu \mathrm{g} / \mathrm{L})$. The concentration of 2-ethyl-1-hexanol in the LRBs analyzed with the FSBs ranged from 0.3 to $2.1 \mu \mathrm{g} / \mathrm{L}$, with a median concentration of $0.9 \mu \mathrm{g} / \mathrm{L}$.

12.4.4. Environmental Samples - Two hundred seventy-six environmental samples, collected from 25 USGS projects nationwide, were analyzed from January through August 2013. Two hundred sixty-two samples were groundwater and 14 were surface water (table 22). Eighteen of the heat purgeable VOCs were detected. 2-Ethyl-1-hexanol was detected in 94 percent of the environmental samples at concentrations similar to the LRBs, ranging in concentration from 0.1 to $1.3 \mu \mathrm{g} / \mathrm{L}$. All values were reported as $<\operatorname{MRL}(<2 \mu \mathrm{g} / \mathrm{L})$. The most frequently detected compounds from this sample set are 1,2,3-TCP, DBCP, and 1,2-DCP. These three fumigant compounds were detected in 8-9 percent of the samples in this dataset. Concentrations detected in samples ranged from 0.005 to $0.526 \mu \mathrm{g} / \mathrm{L}$ for $1,2,3$ TCP, from 0.002 to $0.564 \mu \mathrm{g} / \mathrm{L}$ for $1,2-\mathrm{DCP}$, and from 0.011 to $1.161 \mu \mathrm{g} / \mathrm{L}$ for DBCP. MTBE was detected in 7 percent of the samples, with a maximum concentration of $0.226 \mu \mathrm{g} / \mathrm{L}$. tert-Butyl alcohol, acetonitrile, isopropyl alcohol, and $n$-pentanal were detected in 3-5 percent of the samples. tert-Butyl alcohol, acetonitrile, and isopropyl alcohol were detected frequently in the FSBs (table 21). Cyclohexanone, 1-octanol, dimethoxymethane, butanal, ethyl acetate, 1,1-dichloro-2-propanone, 1,4-dioxane, EDB, and chloropicrin were detected in less than or equal to 2 percent of the samples in this dataset.

12.4.5. Laboratory Matrix Spike (MSPK) — Twenty-three groundwater and seven surface water samples, representing 25 USGS projects nationwide, were submitted as matrix spikes. Upon receipt at the laboratory, the samples were spiked with the CCV standard and held for a minimum of 3 days before analysis. Mean recoveries for the $30 \mathrm{MSPKs}$ ranged from 76 to 116 percent, with RSDs of $\leq 17$ percent (table 23) for all compounds. This compared to mean recoveries for $75 \mathrm{CCV}$ standards (analyzed within the same timeframe as the MSPKs) that ranged from 84 to 111 percent, with RSDs of $\leq 19$ percent (table 19).

Mean recoveries for hexachlorocyclopentadiene in both the groundwater and surface water MSPKs performed within data quality objectives compared to the groundwater and surface water used to determine bias and variability (table 13) during method validation. The recoveries for hexachlorocyclopentadiene ranged from 58 to 121 percent, with a mean recovery of 94 percent for groundwater MSPKs. Mean recovery in surface water MSPKs was 82 percent, ranging from 69 to 89 percent. In the method validation bias and variability study for concentrations spiked from 5 to $50 \mu \mathrm{g} / \mathrm{L}$ mean recoveries for hexachlorocyclopentadiene ranged from 122 to 138 percent in groundwater, and from 131 to 152 percent in surface water.

\subsection{Discussion of Heat Purgeable Method 0-4437 and Historical Methods}

NWQL laboratory schedules are referred to in this section (table 24). Method O-4437 (NWQL LS 4437) refers to the heat purgeable method described in this document using purge-andtrap with simultaneous full scan/SIM GC/MS. NWQL LS 4443 is a subset of NWQL LS 4437, reporting only the fumigant compounds (DBCP, EDB, 1,2-DCP, 1,2,3-TCP, and chloropicrin). Method O-4024 (NWQL LS 4024) (Rose and Sandstrom, 2003) refers to the heat purgeable method for gasoline oxygenates and degradates. Method O-4024 was used to determine four gasoline oxygenate ethers (MTBE, tert-butyl ethyl ether, tert-pentyl methyl ether, and diisopropyl ether) as well as four degradation compounds (acetone, methyl acetate, tert-butyl alcohol, and tert-amyl alcohol). Method O-4024 used heated purge-and-trap with full scan GC/MS. Method O-4437 retained three compounds from Method O-4024: methyl acetate, MTBE, and tert-butyl alcohol. EPA Method 504.1 (NWQL LS 1306) (U.S. Environmental Protection Agency, 1995) was used for the determination of EDB and DBCP, using electron capture GC. Method O-4437 supersedes Methods O-4024 and EPA 504.1. 
12.5.1. Method O-4024 (NWQL LS 4024)-The RLs decreased from Method O-4024 (Rose and Sandstrom, 2003) by a factor of 2 to 4 because the SIM mode was used during analysis in Method O-4437 (NWQL LS 4437). The RL for methyl acetate decreased from $<0.46 \mu \mathrm{g} / \mathrm{L}$ (NWQL LS 4024) to $<0.14 \mu \mathrm{g} / \mathrm{L}$ (NWQL LS 4437). MTBE RL decreased from $<0.06 \mu \mathrm{g} / \mathrm{L}$ (NWQL LS 4024) to $<0.012 \mu \mathrm{g} / \mathrm{L}$ (NWQL LS 4437), and tert-butyl alcohol RL decreased from $<0.8 \mu \mathrm{g} / \mathrm{L}$ (NWQL LS 4024) to $<0.24 \mu \mathrm{g} / \mathrm{L}$ (NWQL LS 4437).

Preservation requirements have changed from Method O-4024 to Method O-4437. Preservation requirements for Method O-4024 required only chilling the sample at $4{ }^{\circ} \mathrm{C} \pm 2{ }^{\circ} \mathrm{C}$. Method O-4437 requires preservation with $\mathrm{HCl}(1: 1)$ to $\mathrm{pH} 2$ and chilling at $4{ }^{\circ} \mathrm{C} \pm 2{ }^{\circ} \mathrm{C}$. The likelihood of converting MTBE to tert-butyl alcohol as a consequence of its preservation with $\mathrm{HCl}(1: 1)$ does not appear to be significant based upon the results of the holding time study (table 15). By day 14 the recovery of MTBE was complete (100 percent); the recovery of tertbutyl alcohol was 94 percent.

12.5.2. EPA Method 504.1 (NWQL LS 1306)—EPA Method 504.1 (NWQL LS 1306) (U.S. Environmental Protection Agency, 1995) has been used for low-level determination of DBCP and EDB. Method O-4437 replaces NWQL LS 1306 by achieving lower reporting levels, with less sample preparation. The RL for DBCP decreases slightly from $<0.03 \mu \mathrm{g} / \mathrm{L}$ using EPA Method 504.1 (NWQL LS 1306) to $<0.020 \mu \mathrm{g} / \mathrm{L}$ using O-4437; the RL for EDB decreases from $<0.018 \mu \mathrm{g} / \mathrm{L}$ to $<0.004 \mu \mathrm{g} / \mathrm{L}$. Sample preparation for NWQL LS 1306 requires extraction of environmental samples, blanks, and calibration standards into hexane, an organic solvent. Sample preparation for NWQL LS 4437 is less hazardous as it involves preparing calibration standards and blanks with water, and placing the $\mathrm{VOC}$ vials into the autosampler. NWQL LS 4443 determines just the fumigant compounds and is identical to NWQL LS 4437. EPA Method 504.1 does not require sample preservation, but Method O-4437 requires preservation with $\mathrm{HCl}$ (1:1) to $\mathrm{pH} 2$.

12.5.3. Method O-4127 (NWQL LS 2020)—Four fumigant compounds, 1,2,3-TCP, 1,2-DCP, DBCP, and EDB, were included from the ambient Method O-4127. RLs for the fumigant compounds on Method O-4127 range from $<0.026$ to $<0.4 \mu \mathrm{g} / \mathrm{L}$. Using Method O-4437 (NWQL LS 4437 or NWQL LS 4443), the RLs have decreased by more than an order of magnitude, ranging from $<0.004$ to $<0.020 \mu \mathrm{g} / \mathrm{L}$.
12.5.4. Comparison of Environmental Samples Determined by Heated Purge Selected Ion Monitoring Mass Spectrometry to Ambient Purge Full Scan Mass Spectrometry for the Fumigant CompoundsAnalyses of several hundred paired environmental samples were determined using both the heated purge and ambient purge methods from July 2013 through February 2014. Seventy-seven samples contained one or more of the fumigant compounds using the heated purge method. These 77 samples were used for the comparison between the heated purge and ambient purge methods (table 25). Concentrations ranged from 0.005 to $0.971 \mu \mathrm{g} / \mathrm{L}$ for 1,2,3-TCP, detected in 42 samples using the heated purge method. However, 1,2,3-TCP was detected in only 14 of 42 samples using the ambient purge method, with concentrations ranging from 0.022 to $0.916 \mu \mathrm{g} / \mathrm{L}$. DBCP concentrations from 43 samples ranged from 0.010 to $1.324 \mu \mathrm{g} / \mathrm{L}$ when analyzed by the heated purge method. DBCP was detected in only 28 of 43 samples when analyzed using the ambient purge method (concentration range from 0.125 to $1.096 \mu \mathrm{g} / \mathrm{L}$ ). EDB was detected in four samples, ranging in concentration from 0.008 to $0.053 \mu \mathrm{g} / \mathrm{L}$ using the heated purge method, but only detected in one sample at $0.057 \mu \mathrm{g} / \mathrm{L}$ using the ambient purge method.1,2-DCP was detected in 47 samples, ranging in concentration from 0.001 to $0.835 \mu \mathrm{g} / \mathrm{L}$ by the heated purge method, but detected in only 25 of 47 samples using the ambient purge method (concentration range from 0.008 to $0.806 \mu \mathrm{g} / \mathrm{L}$ ). Refer to figure 7 for a 1:1 plot comparing the results of the determinations using both the heated purge and ambient purge methods.

\subsection{Results and Discussion of Laboratory and Environmental Samples Determined by Method 0-4436}

12.6.1. Laboratory Continuing Calibration Verification Standards-Mean recoveries for 245 CCV standards, analyzed from January 2013 through June 2014, ranged from 83 to 116 percent, with RSDs $\leq 30$ percent for all ambient purgeable VOCs on Method O-4436 (NWQL LS 4436), excluding bromomethane (table 26). Bromomethane had a mean recovery of 96 percent, with a 46 percent RSD. Chloromethane also demonstrated a higher RSD (23 percent). Bromomethane and chloromethane will be reported with an "E" remark code for an estimated concentration. Recovery for both compounds is altered by the presence of sulfur in samples, making them highly variable. Both are gases at room temperature. 
34541 1,2-Dichloropropane (1,2-DCP)

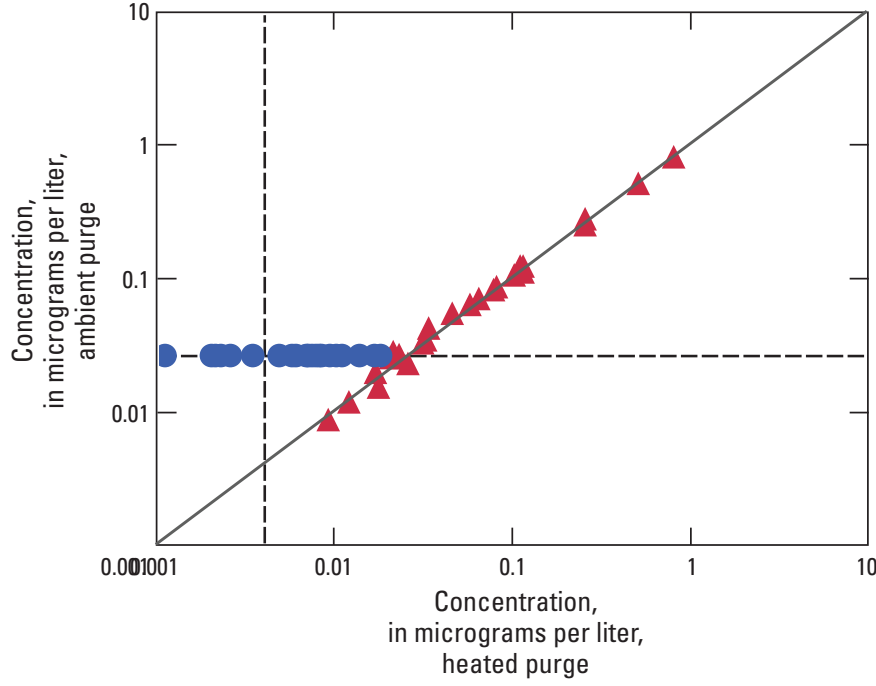

77651 1,2-Dibromoethane (EDB)

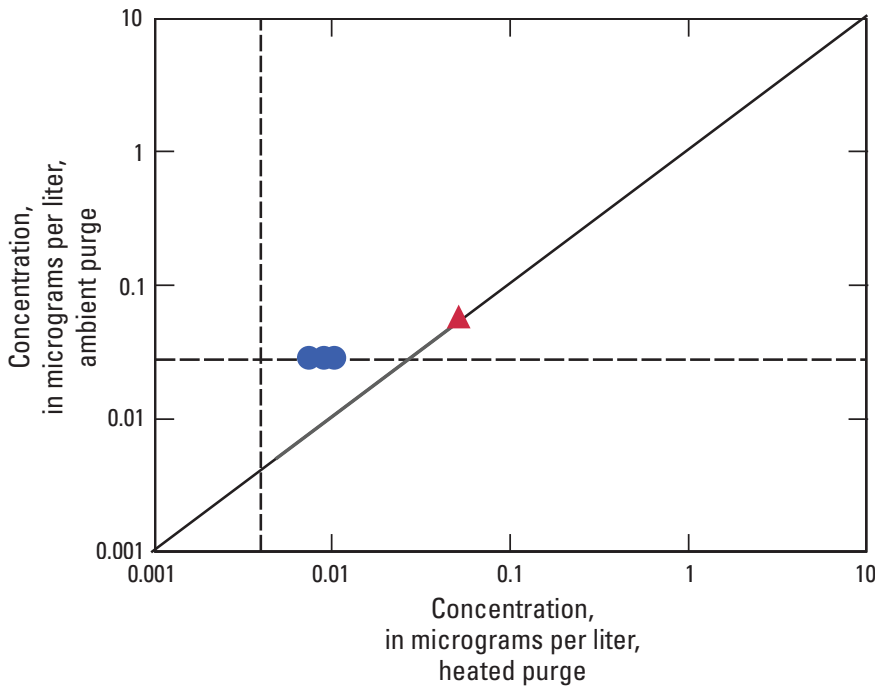

77443 1,2,3-Trichloropropane (1,2,3-TCP)

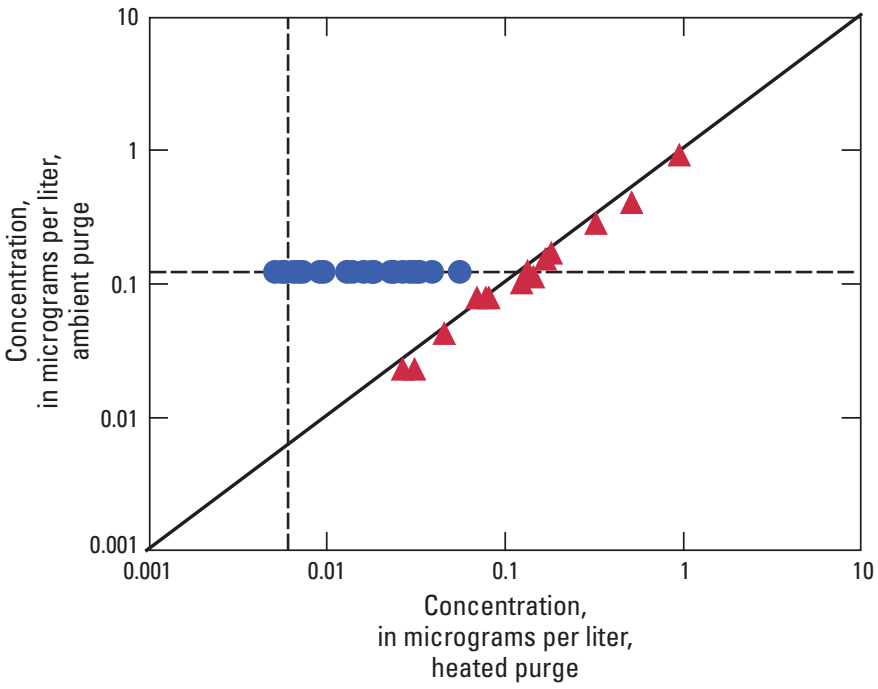

82625 1,2-Dibromo-3-chloropropane (DBCP)

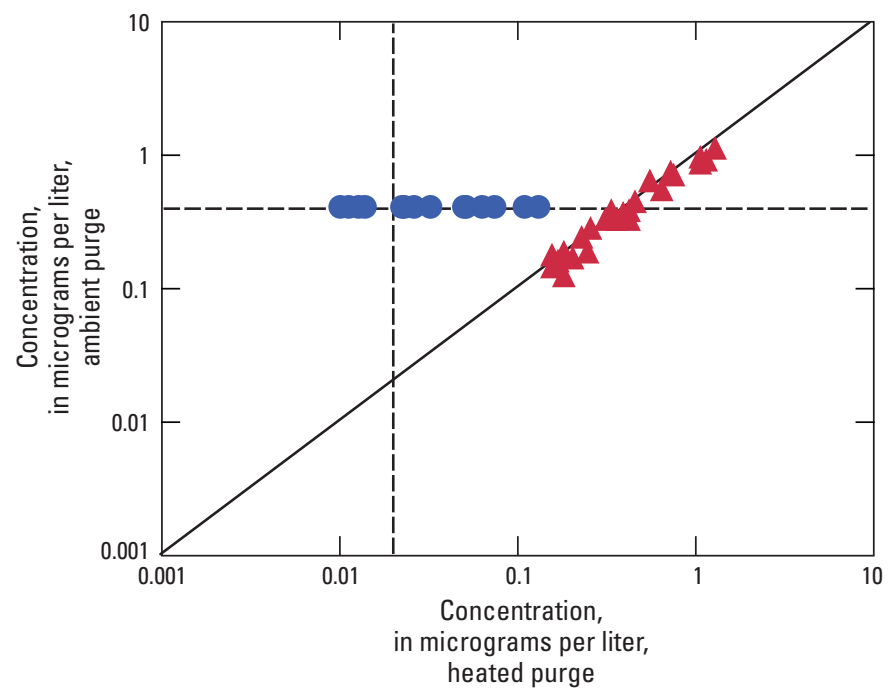

Figure 7. Plot of paired environmental samples determined by the heated purge method compared to the ambient purge method is shown. Red triangles represent detections in both methods, while blue circles represent detections by the heated purge method. Values for nondetections in the ambient purge method are plotted at the reporting level. The solid black line is the line of 1:1 equivalence. The dashed lines represent the reporting levels for each method. Only samples with detections for fumigant compounds are plotted in these figures. See table 25 for a summary of the data.

1,2-Dimethylnaphthalene, 1,6-dimethylnaphthalene, 2,6-di-tert-butyl phenol, and 2-chloronaphthalene began performing poorly in early 2014. Subsequently these four compounds were eliminated from the method on May 1, 2014. Although the mean recoveries for $217 \mathrm{CCV}$ standards range from 87 to 100 percent, the minimum recoveries ranged from 24 to 61 percent. The reporting levels were raised because the compounds were failing identification criteria in the LOQ standard. These compounds are more suited to a method that determines semivolatile compounds.
12.6.2. Laboratory Reagent-Water Blanks (LRB) _ One hundred five LRBs were determined from January through August 2013. Eight ambient purgeable VOCs were detected, including chlorodifluoromethane, 1,2dimethylnaphthalene, 1,6-dimethylnaphthalene, 2,6di-tert-butyl phenol, 2-chloronaphthalene, carbon disulfide, bromomethane, and trichloroethene (table 27).

Chlorodifluoromethane was detected in 13 percent of the LRBs (14 of 105), ranging in concentrations from 0.032 to $0.067 \mu \mathrm{g} / \mathrm{L}$, almost twice the initial $\mathrm{RL}$ of $0.038 \mu \mathrm{g} / \mathrm{L}$. The pattern was not consistent 
throughout an instrument batch run in which all LRBs in a single run would exhibit either decreasing, increasing, or similar concentrations. Chlorodifluoromethane was sporadically detected in the LRBs during a 6-week time period from July through August 2013. The source of the contamination was not identified, but it is suspected that possibly the VOC vials may have been exposed to chlorodifluoromethane during storage. Chlorodifluoromethane is used in refrigerant and air-conditioning applications. It is also used as an aerosol propellant, a blowing agent for foam, and in the production of fluorocarbon resins - particularly, the tetrafluoroethylene monomer (Midgley, and Fisher, 1993, Pubchem, 2014). Only one environmental sample and one FSB were reported with slightly raised reporting levels because of the presence of chlorodifluoromethane in the LRB. Chlorodifluoromethane was not defined as a blanklimited compound since recent data have not shown the same pattern in the LRBs.

Four high priority compounds (1,2-dimethylnaphthalene, 1,6-dimethylnaphthalene, 2,6-di-tert-butyl phenol, and 2-chloronaphthalene) were detected in 2-4 percent of the LRBs, ranging in concentration from 0.06 to $0.28 \mu \mathrm{g} / \mathrm{L}$. Initial RLs, based on the MDLs, range from 0.16 to $0.20 \mu \mathrm{g} / \mathrm{L}$. The LRB detections were caused by carryover contamination after analyzing higher concentration standards. The method was initially developed on a VOCARB ${ }^{\circledR}$ trap and then transferred to another purgeand-trap system with a four phase trap. The VOCARB ${ }^{\circledR}$ trap can be heated to a higher temperature than the four-phase trap, resulting in less carryover contamination. Each system will have to be evaluated to determine how much carryover is typical. Additional COBs may need to be analyzed after a calibration curve to reduce residuals to an acceptable level. These compounds were eliminated from the method for poor performance and carryover contamination issues in May 2014, after acquiring and reviewing about 18 months of data.

Of the remaining compounds detected in the LRBs, carbon disulfide was detected in 3 percent of the LRBs at concentrations less than the RL $(<0.1 \mu \mathrm{g} / \mathrm{L})$, ranging from 0.005 to $0.009 \mu \mathrm{g} / \mathrm{L}$. Residual concentrations of carbon disulfide are commonly observed after analyzing samples with high concentrations of hydrogen sulfide or sulfur dioxide. Bromomethane was detected in 2 percent of the LRBs at concentrations ranging from 0.04 to $0.05 \mu \mathrm{g} / \mathrm{L}$, less than the $\mathrm{RL}(<0.2 \mu \mathrm{g} / \mathrm{L})$. Trichloroethene was detected in one $\mathrm{LRB}$ at a concentration of $0.018 \mu \mathrm{g} / \mathrm{L}$, below the RL of $<0.022 \mu \mathrm{g} / \mathrm{L}$. This occurred following the analysis of a high-concentration sample.

12.6.3. Field-Submitted Blanks - Eighty-nine FSBs, collected from diverse groundwater and surface-water sites across the United States, were submitted from
January through August 2013. These included source solution blanks, equipment blanks, field blanks, and trip blanks. Results are summarized in table 28. Three of the high priority ambient purgeable VOCs were detected, including 1,1-difluoroethane, pentane, and hexane. 1,1-Difluoroethane was detected in 12 percent of the FSBs, at concentrations ranging up to $0.124 \mu \mathrm{g} / \mathrm{L}(\mathrm{RL},<0.022 \mu \mathrm{g} / \mathrm{L}) .1$, 1-Difluoroethane is primarily used as a foam-blowing agent and as an aerosol propellant (Barletta and others, 2011). 1,1-Difluoroethane was not detected in any of the LRBs. Pentane was detected in 3 percent of the FSBs. The maximum concentration for pentane was $0.611 \mu \mathrm{g} / \mathrm{L}(\mathrm{RL},<0.022 \mu \mathrm{g} / \mathrm{L})$. Pentane, a component of crude oil and natural gas condensate, is used as a solvent, an aerosol propellant, and as a blowing agent for polystyrene and polyurethane foams (Lee, 2015). Pentane was not detected in any of the LRBs. Hexane was detected in one FSB at a concentration of $0.023 \mu \mathrm{g} / \mathrm{L}$, just below the RL $(<0.024 \mu \mathrm{g} / \mathrm{L})$. Hexane was not detected in any of the LRBs. Hexane also is a minor component of crude oil and natural gas. It is primarily used as an edible-oil extractant for soybeans, cottonseed, and linseed, and is also used as a solvent and cleaning agent in various industries (Center for Disease Control and Prevention, Agency for Toxic Substances \& Disease Registry, 1999).

Thirteen of the 38 ambient purgeable VOCs retained from Method O-4127 (NWQL LS 2020) (Connor and others, 1998) were detected in FSBs. Toluene, methylene chloride, and chloroform were detected in more than 10 percent of the FSBs (table 28). Toluene was detected in 22 percent of the FSBs, ranging in concentration from 0.02 to $0.18 \mu \mathrm{g} / \mathrm{L}$ (MRL, $<0.02 \mu \mathrm{g} / \mathrm{L}$ ). Methylene chloride was detected in 12 percent of the FSBs, ranging in concentration from 0.07 to $0.31 \mu \mathrm{g} / \mathrm{L}$ (MRL, $<0.04 \mu \mathrm{g} / \mathrm{L}$ ). Chloroform was the third most frequently detected compound, ranging from 0.011 to $0.168 \mu \mathrm{g} / \mathrm{L}$ ( $\mathrm{RL},<0.030$ $\mu \mathrm{g} / \mathrm{L})$ in 11 percent of the FSBs. Carbon disulfide, 1,4-dichlorobenzene, benzene, meta- and para-xylene, MTBE, 1,2,4-trimethylbenzene, bromodichloromethane, ethylbenzene, ortho-xylene, and styrene were also detected in FSBs, in decreasing frequency from 8 to 1 percent.

The ambient purgeable VOCs detected in FSBs for Method O-4436 display similar results to historical data for Method O-4127 reported in Thiros and others (2011, table 7). Toluene was the most frequently detected VOC in source solution blanks (29.7 percent) and field blanks (37.9 percent) sampled from 1996 to 2008 for NAWQA groundwater studies. Chloroform was detected in 19.7 percent of field blanks, followed by meta- and para-xylene (17.2 percent), 1,2,4-trimethylbenzene (15.7 percent), ethylbenzene 
(12.4 percent), styrene ( 9.9 percent), carbon disulfide (9.2 percent), methylene chloride (9.2 percent), benzene (8.9 percent), and ortho-xylene (7.7 percent).

Further long-term monitoring of the FSBs will provide data to assess possible contaminants that may be introduced during sampling, transportation, and analysis. A topical study may provide additional information about possible sources for the high priority ambient purgeable VOCs. Thiros and others (2011) report details of a field study on selected VOCs and wastewater indicator compounds for field blanks and groundwater samples using Method O-4127 (NWQL LS 2020) (Connor and others, 1998).

12.6.4. Environmental Samples-Three hundred thirteen environmental samples, collected from multiple sites nationwide, were analyzed using Method O-4436 (NWQL LS 4436), including 307 groundwater and six surface water samples (table 29).

Nine of the high priority ambient purgeable VOCs were detected in environmental samples, ranging in concentration from 0.013 to $72 \mu \mathrm{g} / \mathrm{L}$. Chlorodifluoromethane and dichlorofluoromethane were the most frequently detected compounds. Chlorodifluoromethane was detected in 3 percent of the samples, with concentrations ranging from 0.046 to $0.607 \mu \mathrm{g} / \mathrm{L}(\mathrm{RL},<0.038 \mu \mathrm{g} / \mathrm{L})$. The median concentration was $0.189 \mu \mathrm{g} / \mathrm{L}$. Chlorodifluoromethane was also detected in 13 percent of the LRBs (table 27) at concentrations ranging from 0.032 to $0.067 \mu \mathrm{g} / \mathrm{L}$, with a median concentration of $0.043 \mu \mathrm{g} / \mathrm{L}$. One groundwater sample analyzed in July 2013 was reported with a raised reporting level of $<0.048 \mu \mathrm{g} / \mathrm{L}$ because chlorodifluoromethane was detected in the LRB. Dichlorofluoromethane was also detected in 3 percent of the samples ( 9 of 313 ). The samples with dichlorofluoromethane were from an industrial area with concentrations from 0.306 to $72 \mu \mathrm{g} / \mathrm{L}$ (RL, <0.05 $\mu \mathrm{g} / \mathrm{L})$. Dichlorofluoromethane was not detected in any LRBs. Seven more ambient purgeable VOCs (1,1-difluoroethane, butane, pentane, 1,2,3,4-tetrahydronaphthalene, 1-chloro1,1-difluoroethane, 2,2-dichloro-1,1,1-trifluoroethane, and 2,6-di-tert-butyl phenol) were detected in 2 percent or less of the environmental samples (table 29).

Thirty of the 38 ambient purgeable VOCs retained from Method O-4127 (NWQL LS 2020) (Connor and others, 1998) were detected in the environmental samples. Chloroform was detected in 28 percent of the samples, ranging in concentration from 0.010 to $21.1 \mu \mathrm{g} / \mathrm{L}(\mathrm{RL},<0.030 \mu \mathrm{g} / \mathrm{L})$. Chloroform was detected in 11 percent of FSBs, ranging from 0.011 to $0.168 \mu \mathrm{g} / \mathrm{L}$ in concentration (table 28). Chloroform was not detected in LRBs (table 27).
Carbon disulfide was detected in 19 percent of the samples, with concentrations ranging from 0.01 to $3.31 \mu \mathrm{g} / \mathrm{L}(\mathrm{RL},<0.10 \mu \mathrm{g} / \mathrm{L})$. Carbon disulfide was detected in 8 percent of FSBs, ranging in concentration from 0.01 to $0.04 \mu \mathrm{g} / \mathrm{L}$. Carbon disulfide was detected in 3 percent of LRBs, with a median concentration of $0.005 \mu \mathrm{g} / \mathrm{L}$.

Tetrachloroethene, trichloroethene, MTBE, cis-1,2dichloroethene, and 1,1-dichloroethane in decreasing frequency were detected in 10 to 6 percent of the samples. MTBE was detected in FSBs, with a 2 percent detection frequency and concentrations ranging from 0.04 to $0.36 \mu \mathrm{g} / \mathrm{L}$ ( $\mathrm{RL},<0.10 \mu \mathrm{g} / \mathrm{L})$. Trichloroethene was detected in an LRB at $0.018 \mu \mathrm{g} / \mathrm{L}(\mathrm{RL}$, $<0.022 \mu \mathrm{g} / \mathrm{L}$ ) because of carryover contamination after analyzing a high concentration sample.

Toluene was detected in 2 percent of the samples, ranging in concentration from 0.02 to $4.82 \mu \mathrm{g} / \mathrm{L}$. Toluene was detected in 22 percent of FSBs, with concentrations ranging from 0.02 to $0.18 \mu \mathrm{g} / \mathrm{L}$ (MRL, $<0.02 \mu \mathrm{g} / \mathrm{L})$. Toluene was not detected in LRBs. The remaining 22 ambient purgeable VOCs were detected in 4 percent or less of the samples (table 29).

12.6.5. Laboratory Matrix Spikes - Twenty-eight environmental samples were fortified with method compounds (MSPKs) at $0.58 \mu \mathrm{g} / \mathrm{L}$ upon receipt at the laboratory. The MSPKs were held for a minimum of 3 days and analyzed within 14 days of the sample collection date. The MSPKs were received and analyzed over an 8-month period. Any compound concentrations detected in the environmental samples were subtracted from the MSPK sample to calculate percent recovery. If detected environmental sample concentrations were greater than ten times the amount spiked, the sample was excluded from the dataset. Chlorodifluoromethane, tetrachloroethene, and trichloroethene had one to three samples excluded as indicated by the number of replicates in table 30 . Mean recoveries for all ambient purgeable VOCs were within data quality objectives of $100 \pm 30$ percent, with RSDs $\leq 30$ percent, except for benzyl chloride, which had a mean recovery of 65 percent (table 30 ).

Mean recovery for benzyl chloride in the MSPKs was 65 percent, ranging from 46 to 104 percent, with a RSD of 21 percent. Twenty-one of the $28 \mathrm{MSPKs}$ had recoveries ranging from 47 to 69 percent for benzyl chloride. CCV standards in instrument batch runs longer than 24 hours show recoveries for benzyl chloride of less than 70 percent by the end of the run. The holding time study shows benzyl chloride degraded from 80 percent recovery on day zero to 33 percent on day 14, and 7 percent on day 28 (table 17). Benzyl chloride rapidly undergoes hydrolysis in water. The hydrolysis half-life in water at $\mathrm{pH} 7$ and $25^{\circ} \mathrm{C}$ is estimated at 9.48 hours 
(Environment Canada, 2009). Benzyl chloride was eliminated from the method because of low recoveries in the holding time study.

Mean recoveries ranged from 74 to 111 percent, with RSDs $\leq 28$ percent (table 30 ) for the remaining ambient purgeable VOCs. Three of the 28 environmental samples showed low recoveries for specific compounds. Two of the three samples were from the same geographical area. 1,3-Butadiene and 2,6-di-tert-butyl phenol had low recoveries in three MSPK samples. Styrene had low recoveries in two MSPK samples. The dimethylnaphthalene isomers and 1,2,4-trimethylbenzene had low recoveries ranging from 41 to 49 percent in one MSPK sample.

Mean recovery for 1,3-butadiene was 87 percent with a RSD of 26 percent. Recoveries for three of the 28 MSPKs have recoveries ranging from 16 to 46 percent. The holding time study in VBW showed 1,3-butadiene was stable with 91 percent recovery on day 14 (table 17).

2,6-Di-tert-butyl phenol had low recoveries in three MSPKs, ranging from 21 to 48 percent. The holding time study showed 2,6-di-tert-butyl phenol with 81 percent recovery on day 14 .

Styrene had a mean recovery of 83 percent in MSPK samples, ranging from 5 to 105 percent recovery, with 28 percent RSD. Two of the MSPKs had recoveries between 5 and 8 percent. Recovery of styrene in the holding time study in VBW was 94 percent on day 14, with 1 percent RSD.

Mean recovery of hexane was 74 percent, with 11 percent RSD and recoveries ranging from 59 to 90 percent. Nine of the 28 MSPKs had recoveries between 59 and 68 percent. The holding time study showed hexane degraded from 89 percent recovery on day zero to 53 percent by day 14 in VBW. The calculated ASTM holding time is 6 days (table 18). CCVs also were observed to degrade with longer instrument runs. Mean recovery in $245 \mathrm{CCVs}$ (table 26) was 83 percent, ranging from 60 to 102 percent with a RSD of 10 percent. The recoveries observed in the MSPKs mimic the behavior observed in the holding time study and the CCVs. Hexane results are reported with an "E" remark code because of poor performance seen during method validation.

\subsection{Discussion of Method 0-4436 (NWQL LS 4436) and Method 0-4127 (NWOL LS 2020)}

The ambient purgeable method, O-4436 (NWQL LS 4436), described in this report is a modification of Method O-4127 (NWQL LS 2020) (Connor and others, 1998). Eleven high priority compounds were added, 38 compounds were retained, 4 compounds were transferred to the heat purgeable method, and 43 compounds were discontinued. Minor modifications were made to Method O-4127:

- 1,2-DCA- $d_{4}$ was changed from a surrogate standard compound in NWQL LS 2020 to an internal standard compound for the halogenated alkanes and vinyl chloride in Method O-4436 (NWQL LS 4436).

- Fifteen compounds are now assigned to internal standard compound 1,2-DCA- $d_{4}$ instead of fluorobenzene. Ambient purgeable VOCs assigned to $1,2-\mathrm{DCA}-d_{4}$ in Method O-4436 include 1,1,1,2-tetrachloroethane, 1,1,1-trichloroethane, 1,1,2-trichloroethane, 1,1-dichloroethane, 1,2-dichloroethane, bromochloromethane, bromomethane, chloromethane, methylene chloride, tetrachloromethane, vinyl chloride, and the trihalomethanes (bromodichloromethane, bromoform, chloroform, and dibromochloromethane).

12.7.1. Statistical Comparison of Continuing Calibration Verification Standards Determined by 0-4436 (NWQL LS 4436) and 0-4127 (NWQL LS 2020) - Thirty-eight ambient purgeable VOCs and two surrogate standard compounds were retained in Method O-4436 from Method O-4127 (NWQL LS 2020) (Connor and others, 1998). A comparison of CCV standards determined using Method O-4436 (NWQL LS 4436) and Method O-4127 (NWQL LS 2020) was done to determine if there were a statistical difference between the two methods. Results were retrieved from the NWQL's laboratory information management system for data from January 2013 through June 2014 for NWQL LS 4436 and NWQL LS 2020. There were 245 CCV standards for NWQL LS 4436 (table 26) and 224 CCV standards for NWQL LS 2020.

Median recoveries of the ambient purgeable VOCs in CCV standards and their differences between NWQL LS 4436 and NWQL LS 2020 are summarized in table 31 and figure 8. Median recoveries for NWQL LS 4436 CCV standards ranged from 84 to 116 percent while median recoveries for NWQL LS 2020 ranged from 86 to 112 percent. Median recoveries are generally higher in NWQL LS 2020 compared to NWQL LS 4436 although recovery differences were small. The differences in median recovery between NWQL LS 4436 and NWQL LS 2020 for all ambient purgeable VOCs ranged from -24 to 10 percent, which is consistent with the overall data quality objectives of the method during development (recovery of $100 \pm 30$ percent).

The median recoveries for each method were compared using the Wilcoxon rank sum test (Helsel and Hirsch, 2002) to determine whether the median recoveries were the same for both methods. The differences in recovery were not statistically significant $(p>0.05)$ 
32101 Bromodichloromethane

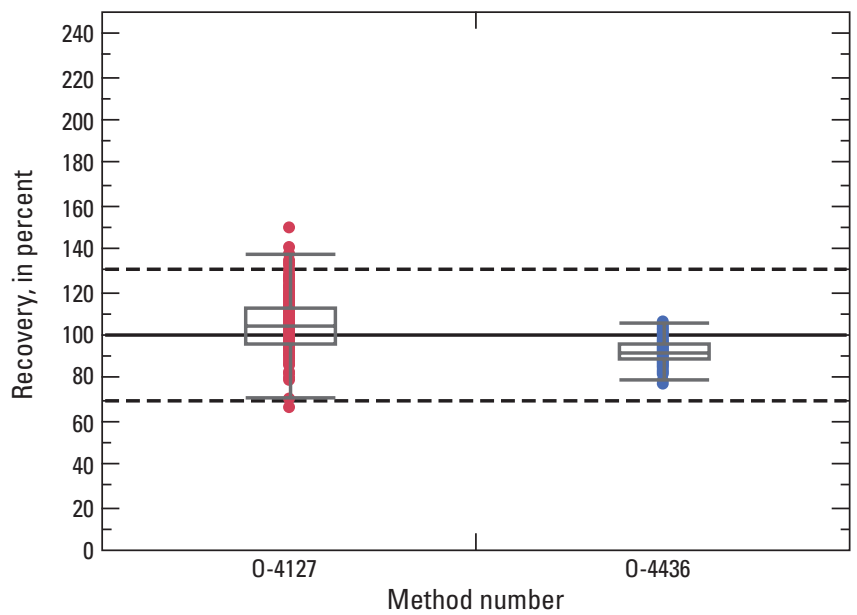

32103 1,2-Dichloroethane

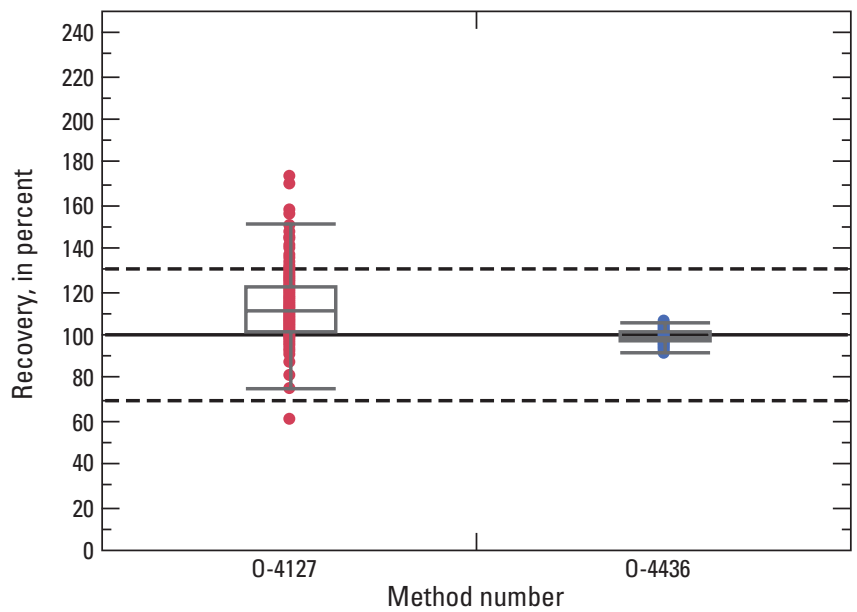

32105 Dibromochloromethane

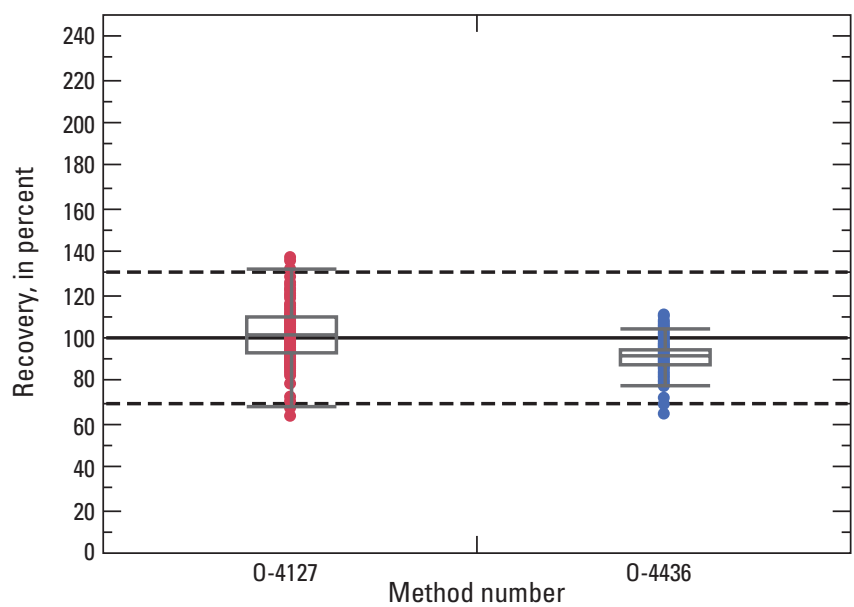

32102 Tetrachloromethane

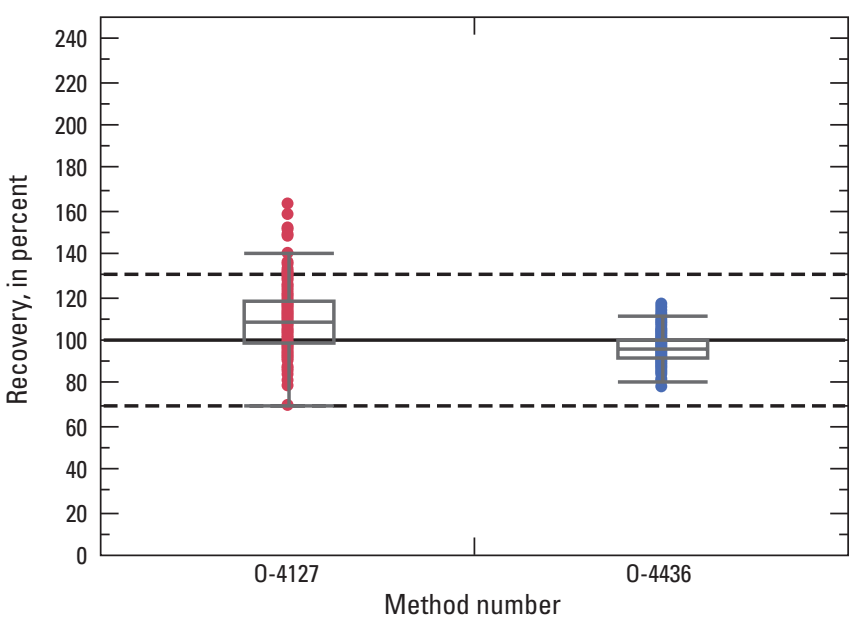

32104 Bromoform

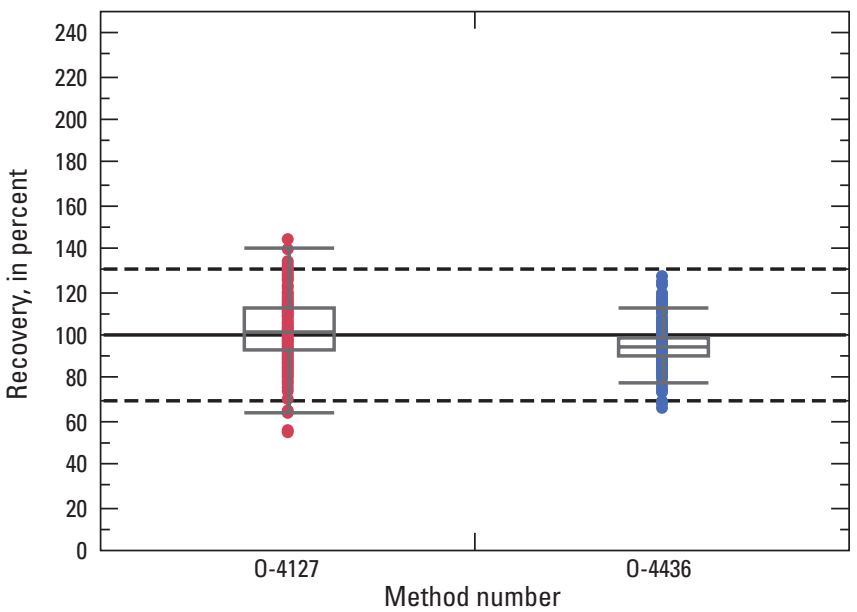

32106 Chloroform

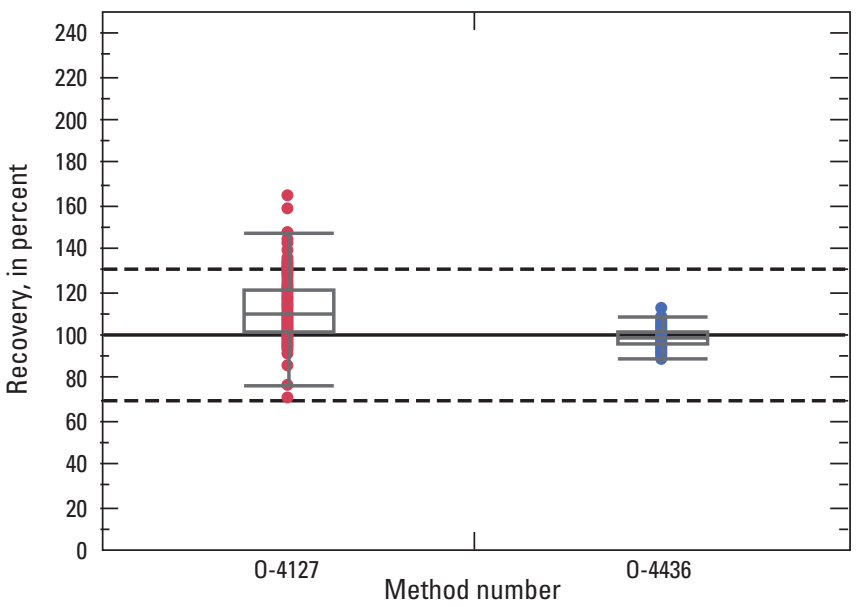

Figure 8. Comparison of continuing calibration verification standard recoveries for ambient purgeable volatile organic compounds determined by Methods 0-4127 ( $\mathrm{N}=229)$ and 0-4436 $(\mathrm{N}=245)$, from January 2013 through June 2014. Compounds assigned to internal standard fluorobenzene are shown in red; compounds assigned to internal standard 1,2-dichloroethane- $d_{4}$ are shown in blue. Dashed lines at 70 and 130 percent recovery represent the data quality objectives used to evaluate the data. 

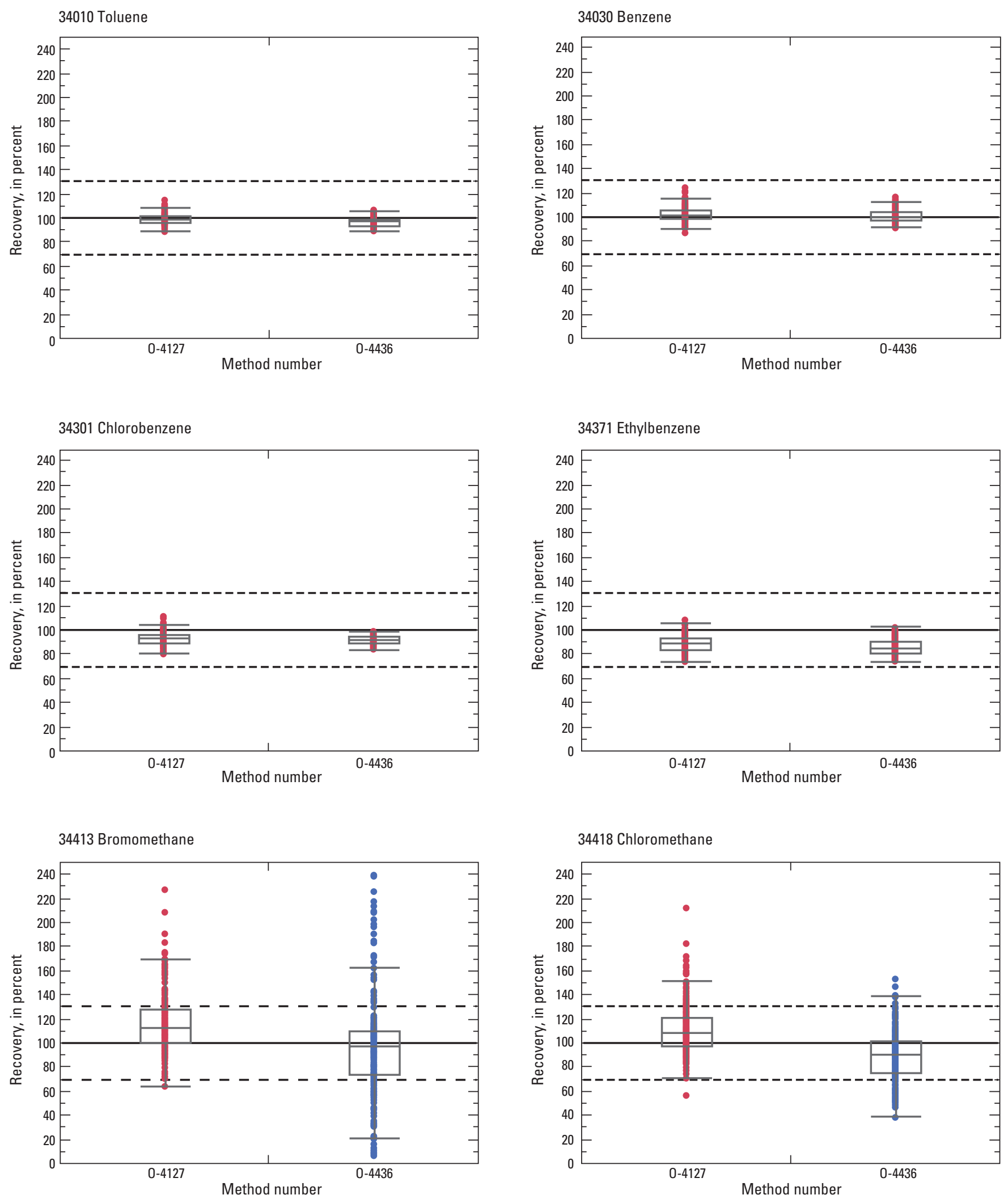

Figure 8. Comparison of continuing calibration verification standard recoveries for ambient purgeable volatile organic compounds determined by Methods 0-4127 ( $N=229$ ) and 0-4436 ( $=245)$, from January 2013 through June 2014. Compounds assigned to internal standard fluorobenzene are shown in red; compounds assigned to internal standard 1,2-dichloroethane- $d_{4}$ are shown in blue. Dashed lines at 70 and 130 percent recovery represent the data quality objectives used to evaluate the data.-Continued 
34423 Methylene chloride

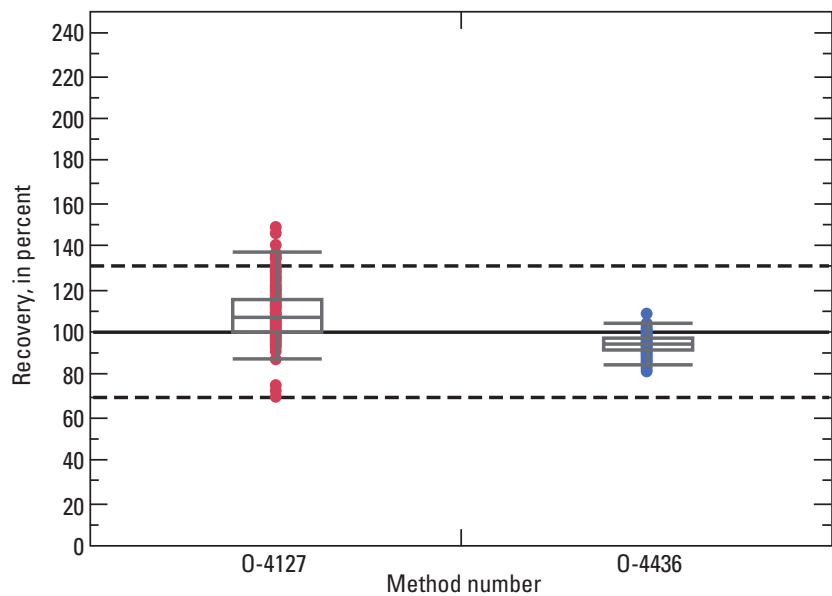

34496 1,1-Dichloroethane

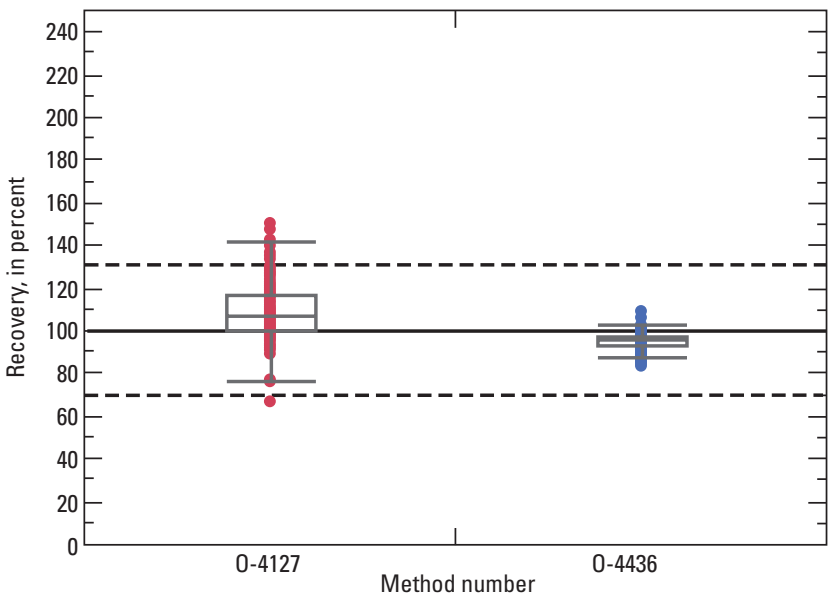

34506 1,1,1-Trichloroethane

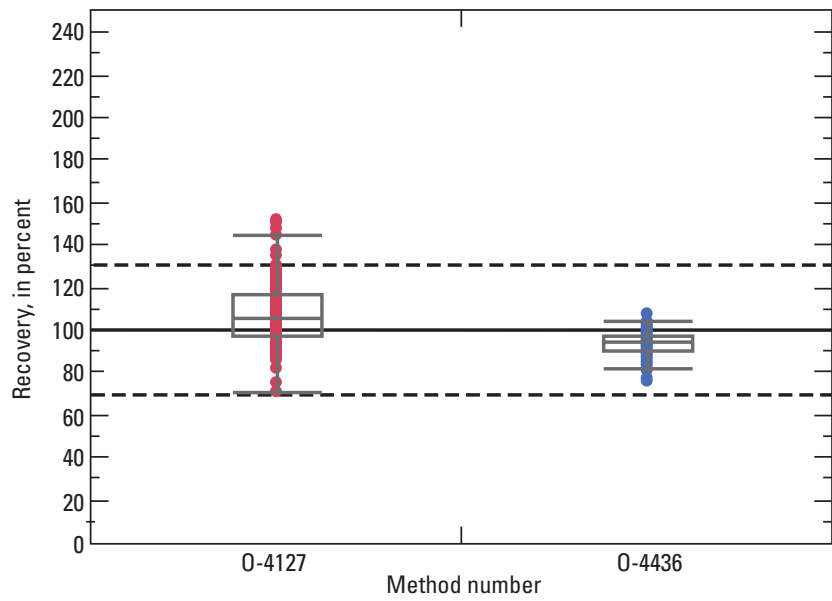

34475 Tetrachloroethene

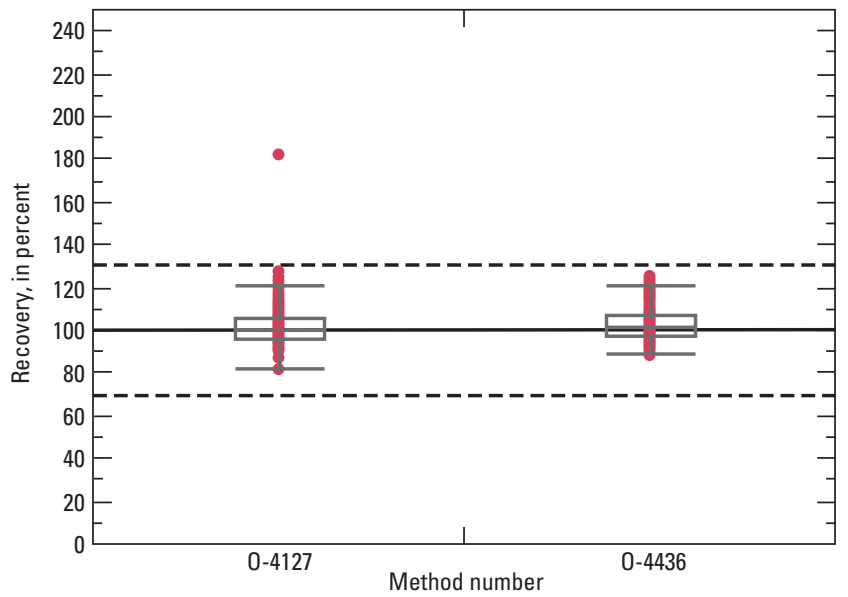

34501 1,1-Dichloroethene

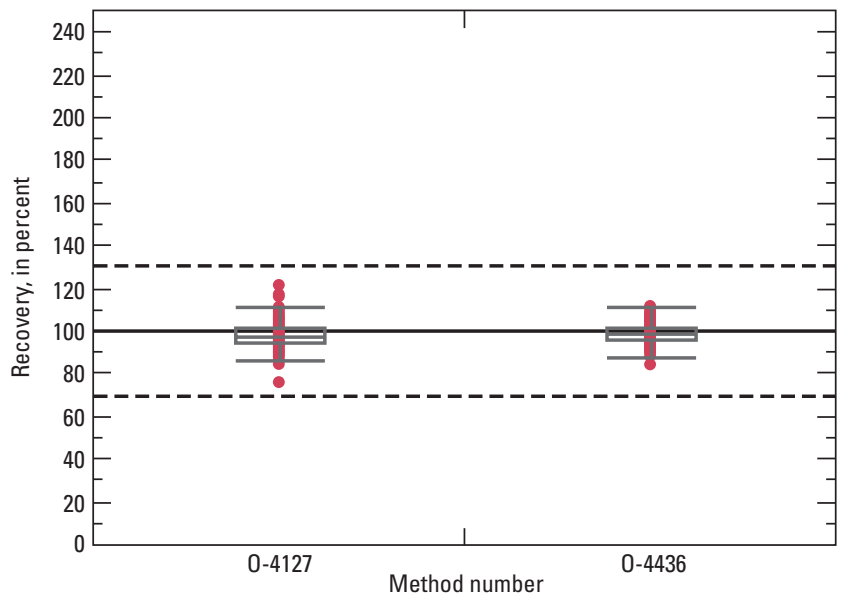

34511 1,1,2-Trichloroethane

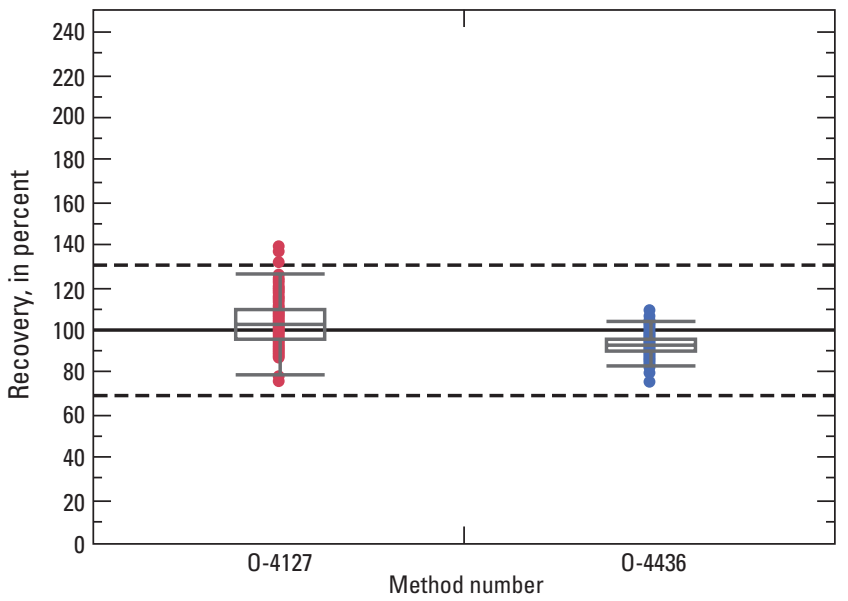

Figure 8. Comparison of continuing calibration verification standard recoveries for ambient purgeable volatile organic compounds determined by Methods 0-4127 ( $N=229)$ and 0-4436 $(\mathrm{N}=245)$, from January 2013 through June 2014. Compounds assigned to internal standard fluorobenzene are shown in red; compounds assigned to internal standard 1,2-dichloroethane- $d_{4}$ are shown in blue. Dashed lines at 70 and 130 percent recovery represent the data quality objectives used to evaluate the data.-Continued 
34536 1,2-Dichlorobenzene

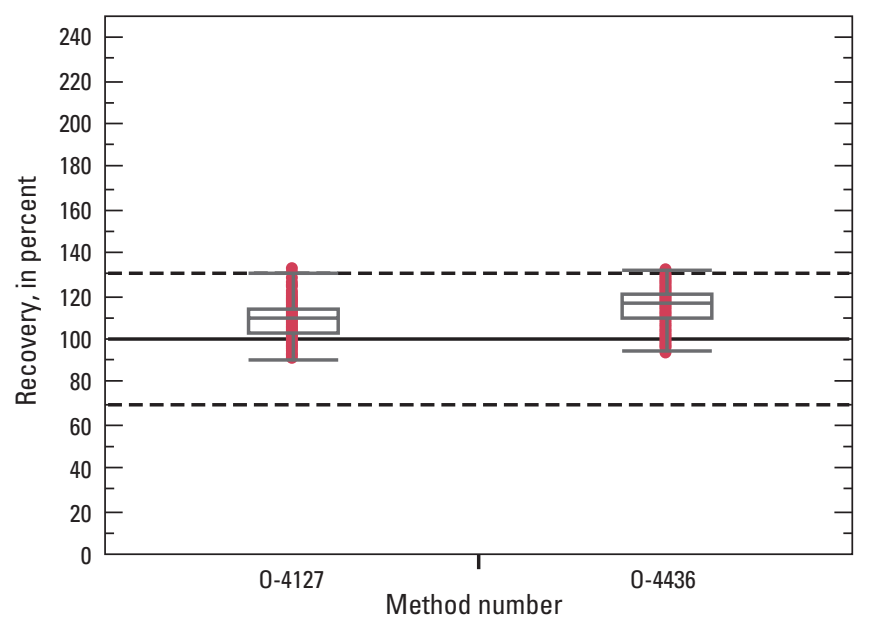

34551 1,2,4-Trichlorobenzene

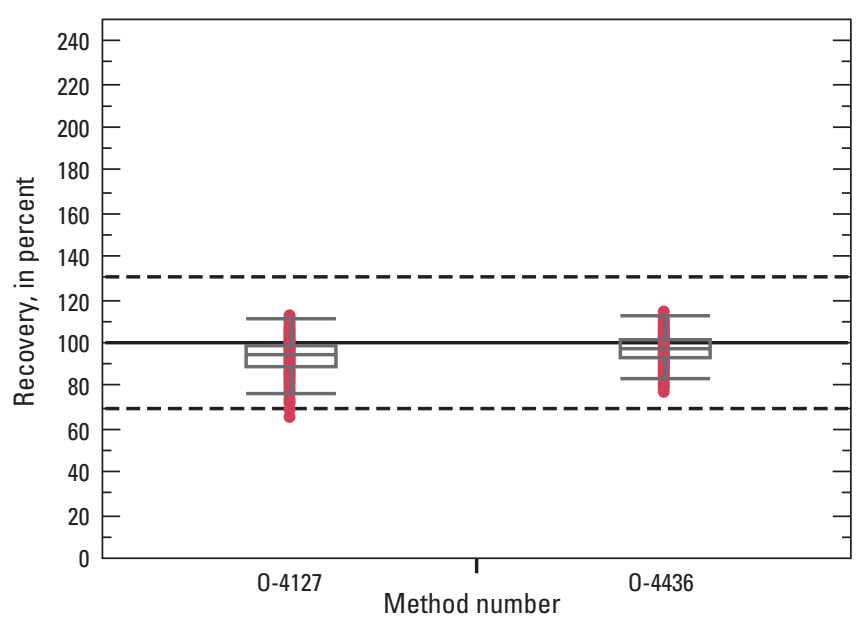

34696 Naphthalene

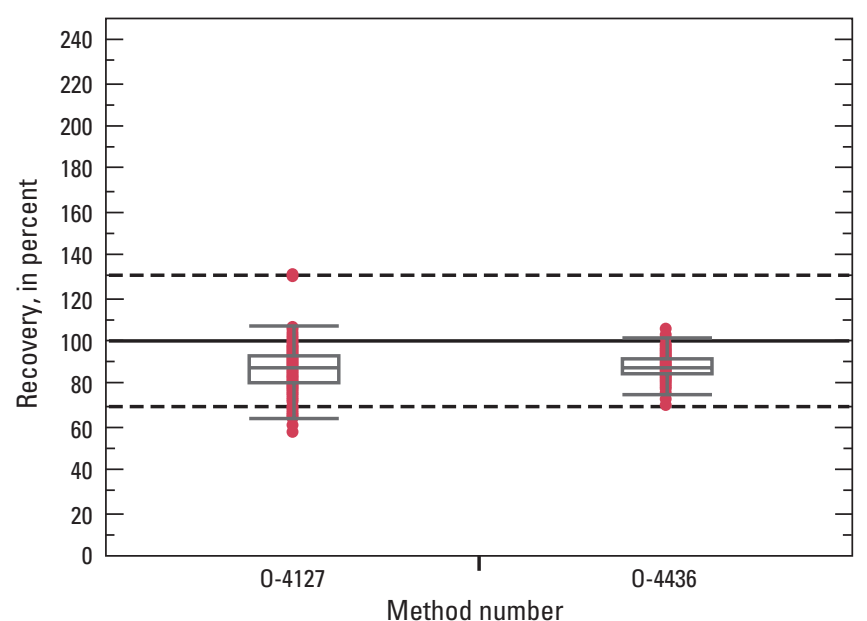

34546 trans-1,2-Dichloroethene

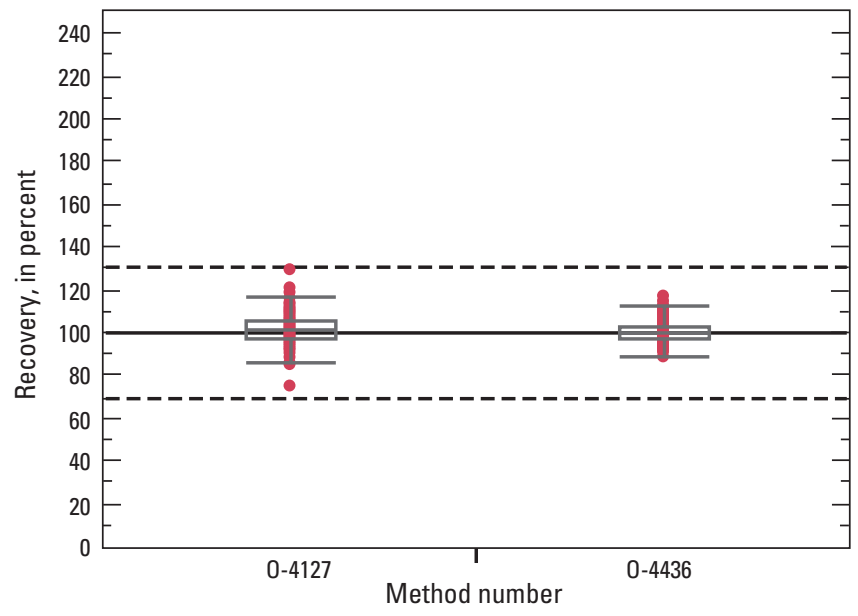

34571 1,4-Dichlorobenzene

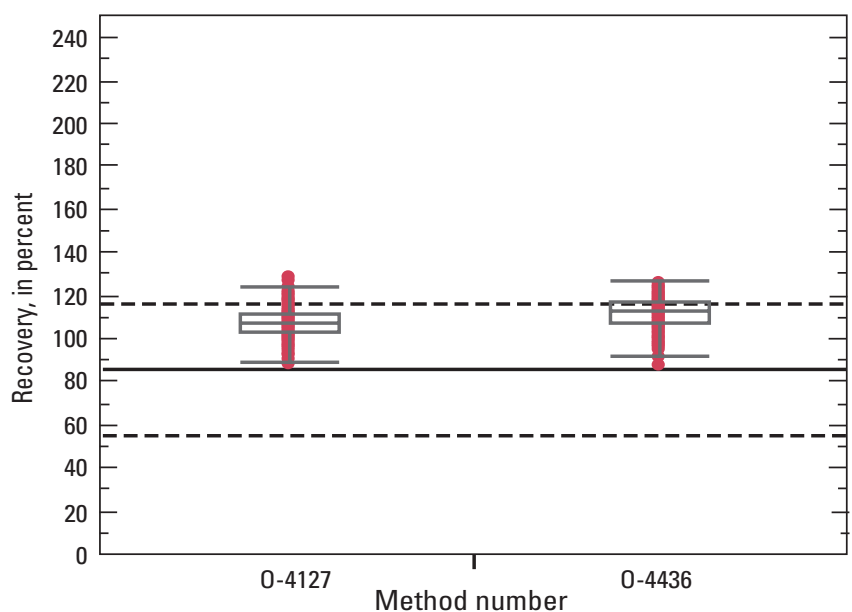

34699 trans-1,3-Dichloropropene

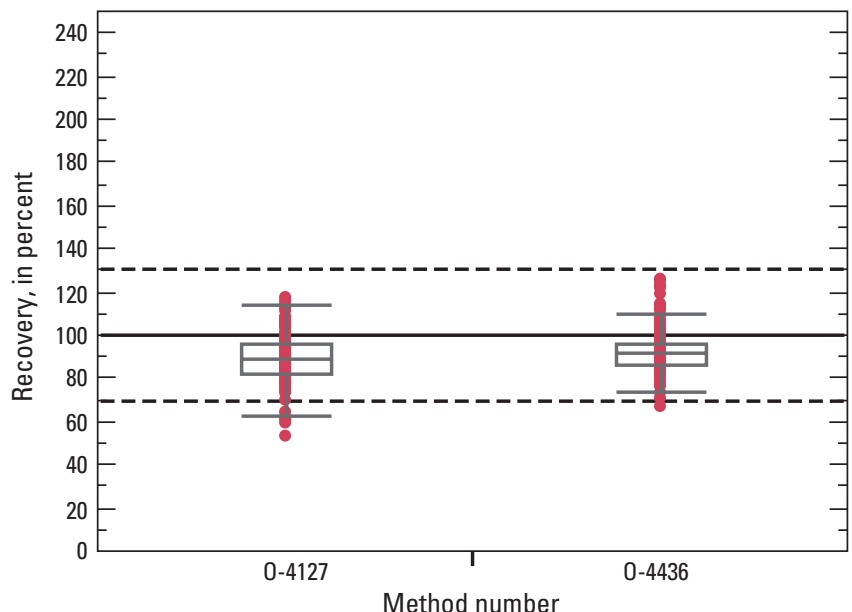

Figure 8. Comparison of continuing calibration verification standard recoveries for ambient purgeable volatile organic compounds determined by Methods 0-4127 ( $\mathrm{N}=229)$ and 0-4436 $(\mathrm{N}=245)$, from January 2013 through June 2014. Compounds assigned to internal standard fluorobenzene are shown in red; compounds assigned to internal standard 1,2-dichloroethane- $d_{4}$ are shown in blue. Dashed lines at 70 and 130 percent recovery represent the data quality objectives used to evaluate the data.-Continued 
34704 cis-1,3-Dichloropropene

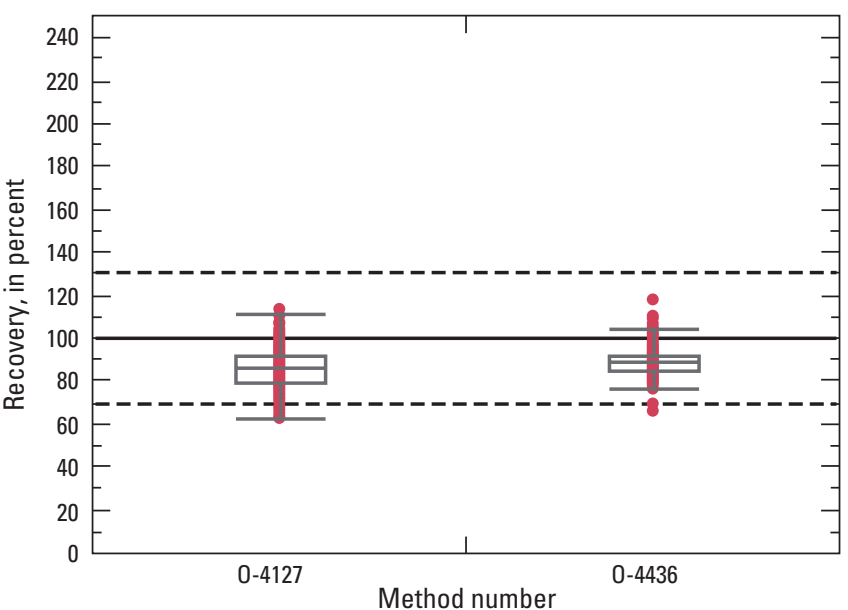

39180 Trichloroethene

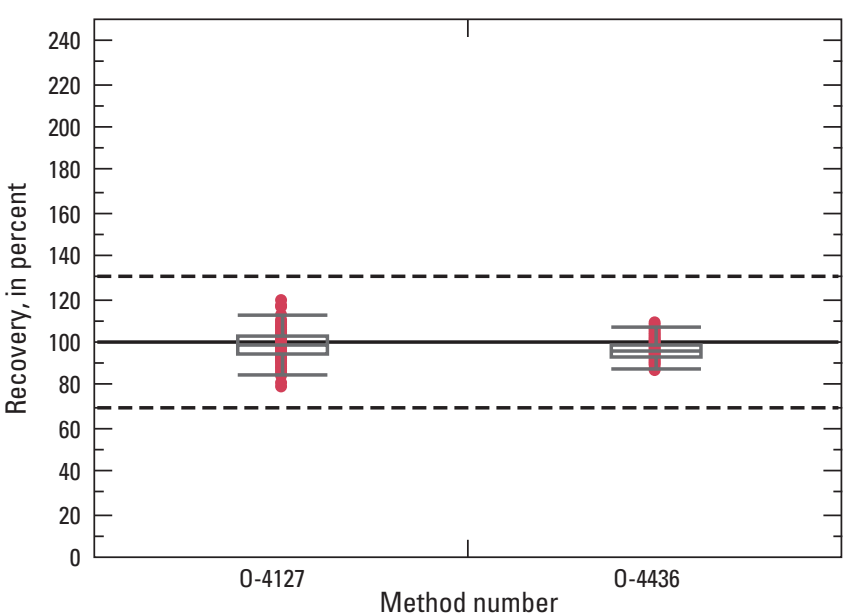

77093 cis-1,2-Dichloroethene

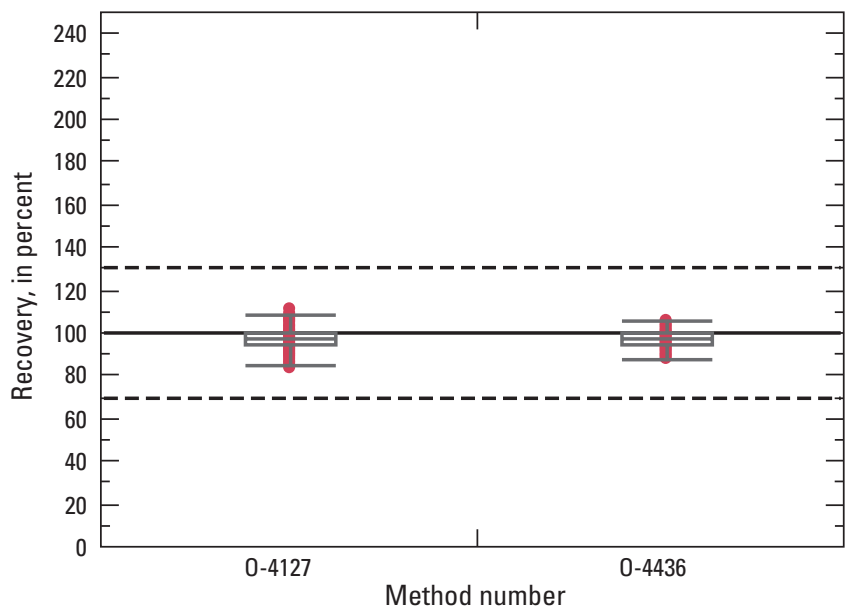

39175 Vinyl chloride

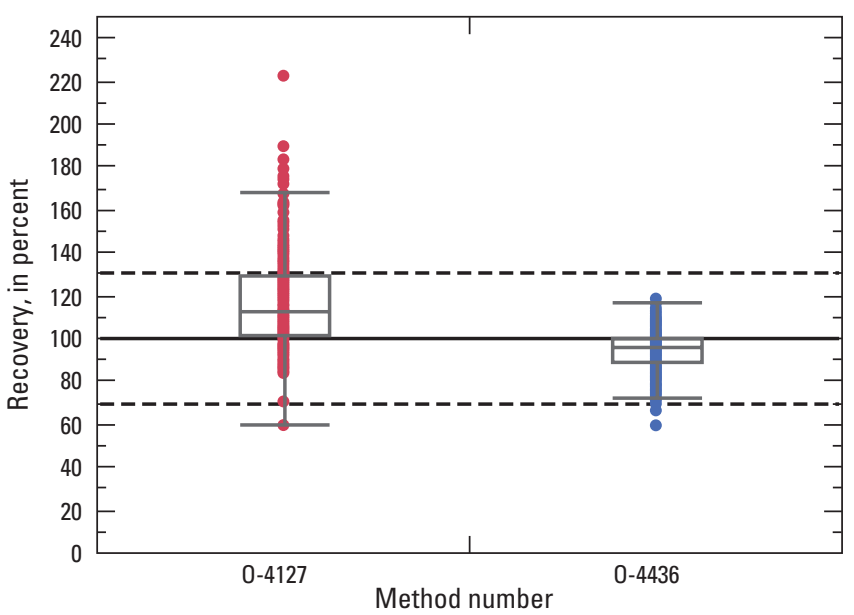

77041 Carbon disulfide

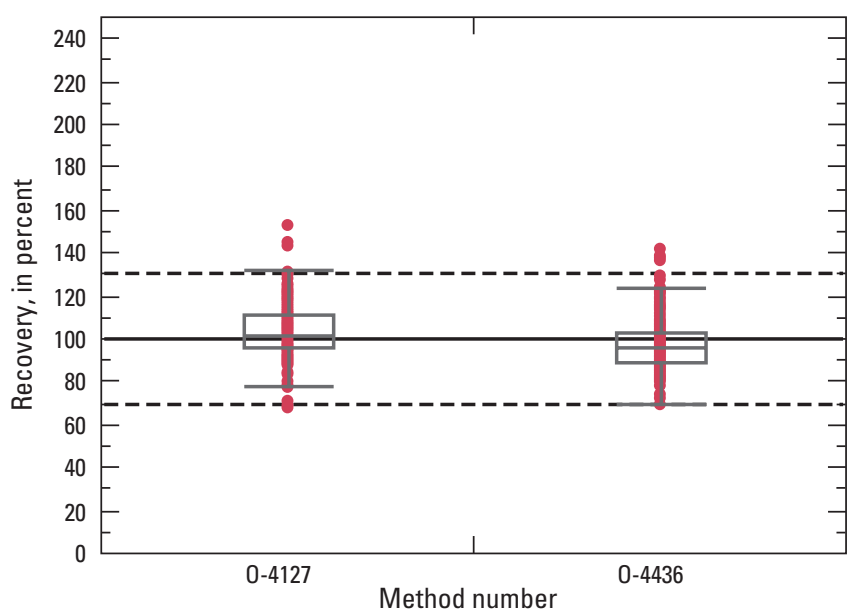

77128 Styrene

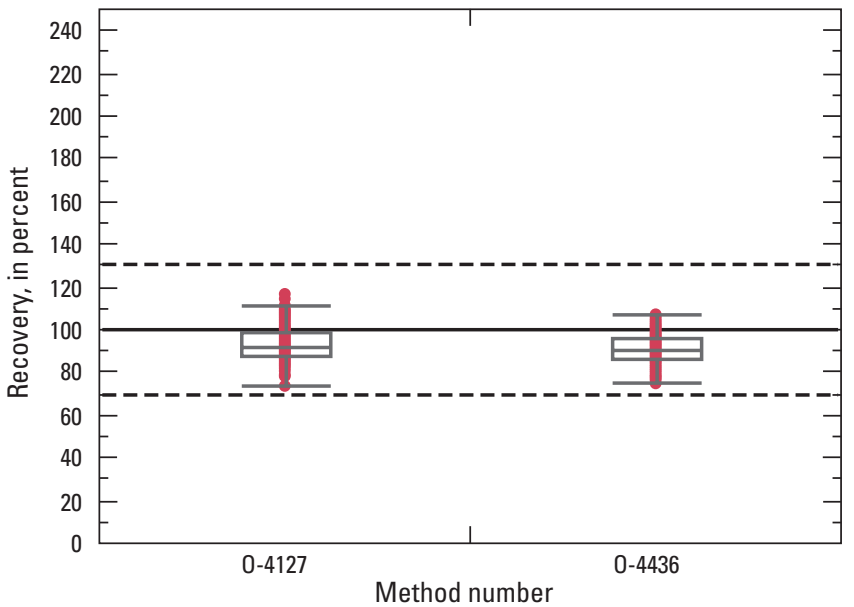

Figure 8. Comparison of continuing calibration verification standard recoveries for ambient purgeable volatile organic compounds determined by Methods 0-4127 ( $\mathrm{N}=229)$ and 0-4436 $(\mathrm{N}=245)$, from January 2013 through June 2014. Compounds assigned to internal standard fluorobenzene are shown in red; compounds assigned to internal standard 1,2-dichloroethane- $d_{4}$ are shown in blue. Dashed lines at 70 and 130 percent recovery represent the data quality objectives used to evaluate the data.-Continued 

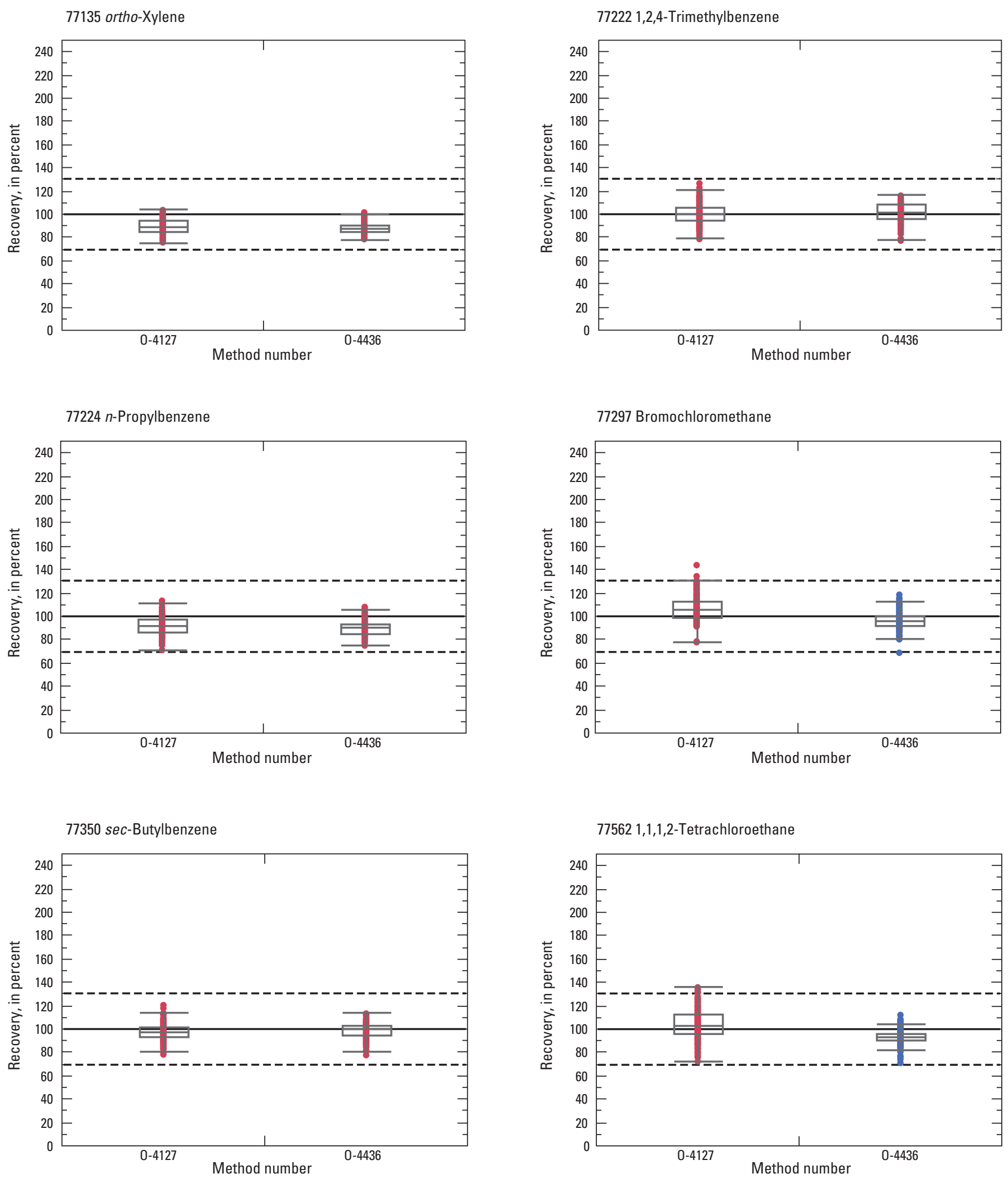

Figure 8. Comparison of continuing calibration verification standard recoveries for ambient purgeable volatile organic compounds determined by Methods 0-4127 ( $\mathrm{N}=229$ ) and 0-4436 ( $\mathrm{N}=245)$, from January 2013 through June 2014. Compounds assigned to internal standard fluorobenzene are shown in red; compounds assigned to internal standard 1,2-dichloroethane- $d_{4}$ are shown in blue. Dashed lines at 70 and 130 percent recovery represent the data quality objectives used to evaluate the data.-Continued 
78032 tert-Butyl methyl ether (MTBE)

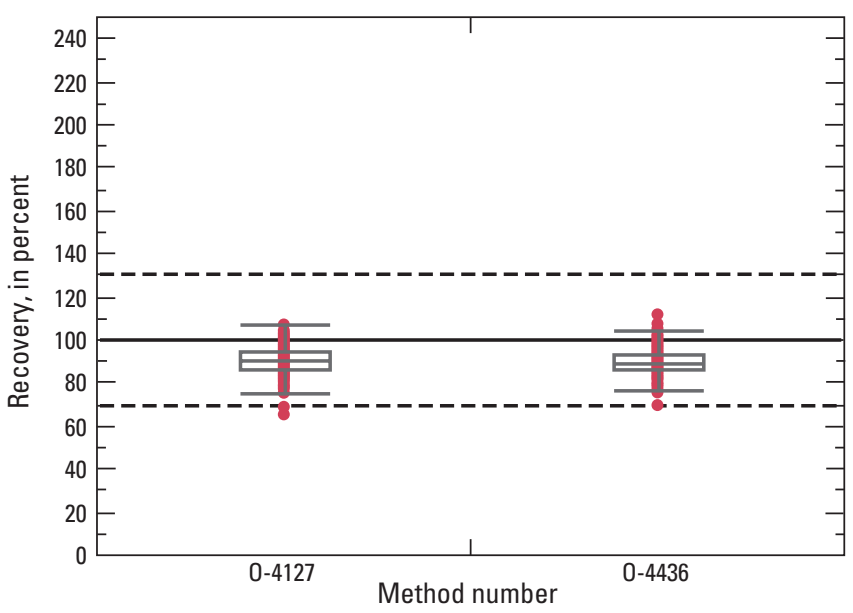

85795 meta- and para- Xylene

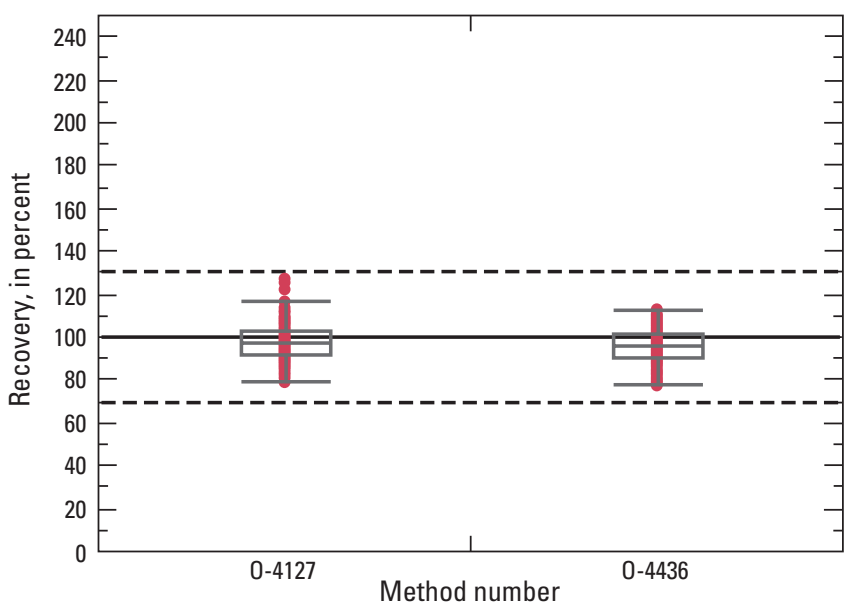

Figure 8. Comparison of continuing calibration verification standard recoveries for ambient purgeable volatile organic compounds determined by Methods 0-4127 ( $\mathrm{N}=229)$ and 0-4436 ( $\mathrm{N}=245)$, from January 2013 through June 2014. Compounds assigned to internal standard fluorobenzene are shown in red; compounds assigned to internal standard 1,2-dichloroethane- $d_{4}$ are shown in blue. Dashed lines at 70 and 130 percent recovery represent the data quality objectives used to evaluate the data.-Continued

for ortho-xylene, 1,1-dichloroethene, MTBE, naphthalene, and cis-1,2-dichloroethene. These five compounds are referenced to internal standard compound fluorobenzene in both methods. However, the differences were statistically significant $(p<0.05)$ for 33 of the 38 ambient purgeable VOCs and the two surrogate standard compounds. The magnitude of these differences in recovery was less than 15 percent for most of the ambient purgeable VOCs (35 of 38) and the two surrogate standard compounds. Bromochloromethane, chloromethane, and vinyl chloride had relatively larger differences in median recovery, ranging from -18 to -24 percent. Chloromethane and vinyl chloride are gases at room temperature. Chloromethane is reported with an "E" remark code because of higher variability. Overall, the small differences in median recovery of the ambient purgeable VOCs in CCV standards show that there were no major differences in method operation and performance because of the change in the internal standard compound.

Variability of recoveries of the ambient purgeable VOCs in CCV standards and their differences between Methods O-4436 and O-4127 (Connor and others, 1998) are summarized in table 32 and figure 8 . Variability measured by the standard deviation of recovery of CCV standards was similar for most ambient purgeable VOCs. Differences in standard deviation between methods were less than or equal to 10 percent for 34 of the 38 compounds. Overall, variability was consistently smaller in Method O-4436 as indicated by negative differences in variability of Method O-4436 minus Method O-4127.
1,2-Dichloroethane, bromomethane, vinyl chloride, and chloromethane had differences in standard deviations, ranging from -13 to 21 percent.

The standard deviations for methods were compared using the Levene and F tests to test whether the variability was the same in each method (National Institute of Standards and Technology, 2000). There was no significant difference in the variability in 11 of the 38 ambient purgeable VOCs in the Levene test of standard deviations $(p>0.05)$. There were statistically significant $(p<0.05)$ differences in variability for the remaining 27 ambient purgeable VOCs and the two surrogate standard compounds, but the differences in variability were small-less than 15 percent for 26 of the 27 compounds. Bromomethane had a difference of 21 percent in variability between NWQL LS 4436 and NWQL LS 2020, which is within the data quality objectives. Bromomethane is a gas at room temperature and is reported with an " $E$ " remark code because of interferences from sulfur. Overall, the small differences in the standard deviation confirm that there are no major differences in the operation of the method because of the internal standard compound change.

12.7.2. Comparison of Paired Environmental Samples Determined by 0-4436 (NWQL LS 4436) and 0-4127 (NWQL LS 2020)_About 70 samples were analyzed using both NWQL LS 4436 and NWQL LS 2020. Because of the small sample size, there are only a few detections for comparison. Chloroform had the most detections. A 1:1 plot (fig. 9) of all detected halogenated alkanes shows good comparison between the two methods. 


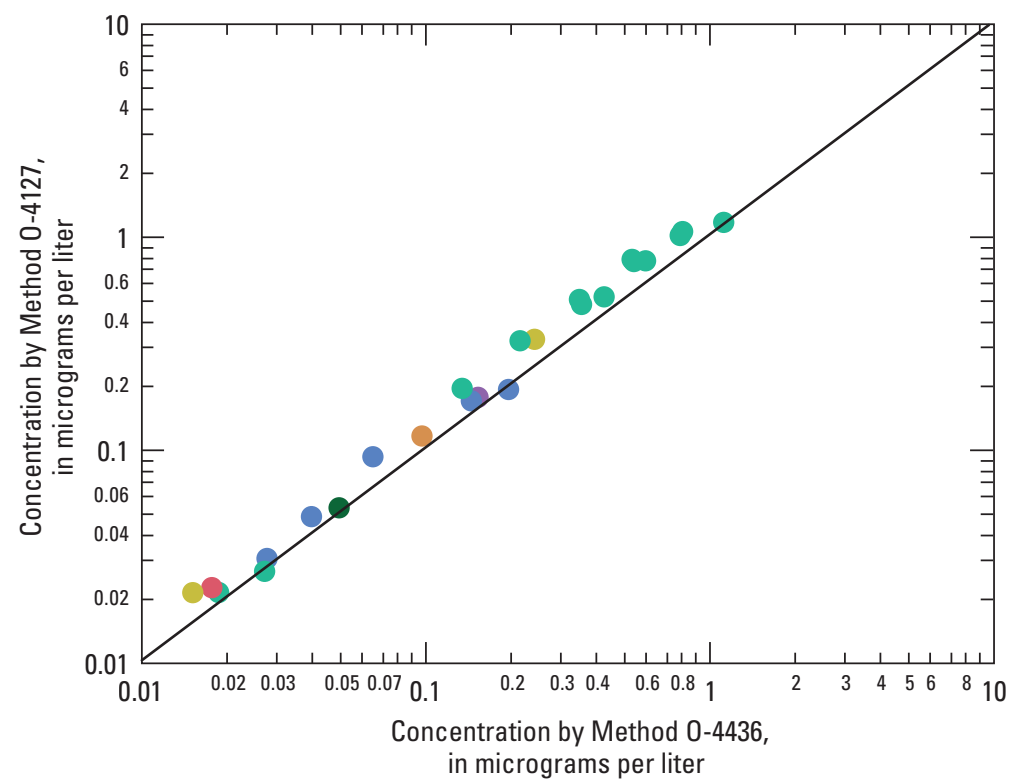

\section{EXPLANATION}

1,1,1-Trichloroethane

- 1,2-Dichloroethane

- Bromodichloromethane

- Bromoform

Chloroform

- Methylene chloride

Tetrachloromethane

Figure 9. Concentrations of volatile organic compounds determined in paired environmental samples determined by ambient purge Method 0-4436 and calculated with 1,2-dichloroethane- $d_{4}$ as the internal standard compound and by ambient purge Method 0-4127 and calculated with fluorobenzene as the internal standard compound. Solid line shows the 1:1 line of equality.

\section{Summary and Conclusions}

The U.S. Geological Survey National Water Quality Laboratory (NWQL) developed two new analytical methods for the determination of 37 heat purgeable (Method O-4437) and 49 ambient purgeable (Method O-4436) volatile organic compounds (VOCs). The methods are applicable to unfiltered water-matrix types, including groundwater, surface water, and drinking water. Performance may vary for other matrices, including wastewater effluents, landfill leachates, and urban runoff storm samples. Most sample data in this report were collected from groundwater and surface water.

Method compounds are determined in unfiltered water samples, collected in a 40-millilter $(\mathrm{mL})$ borosilicate vials with Teflon-faced septa, preserved to $\mathrm{pH} 2$ with a 1:1 hydrochloric acid:water solution ( $\mathrm{HCl}[1: 1])$, and chilled at 4 degrees Celsius \pm 2 degrees Celsius until analysis. Ascorbic acid is added if free chlorine is present. Samples are analyzed within 14 days of sampling. The sample vials are loaded directly onto an autosampler. For each sample $25 \mathrm{~mL}$ is transferred to the sparge vessel of the purge-and-trap concentrator. The sample is purged with helium. Compounds are trapped onto a sorbent trap, and desorbed into the gas chromatograph. Compounds exiting the gas chromatography (GC) column are transferred to the mass spectrometer, and ionized by electron impact. The heat purgeable VOCs are determined in the simultaneous full scan/selected ion monitoring mode, whereas the ambient purgeable VOCs are determined in the full scan mode. Specific ions are monitored for each compound. All compounds are quantitated relative to a specific internal standard compound.
Performance for each method was tested by spiking replicates of a chosen groundwater, surface water, and reagent water at two concentrations. Three separate analytical batches were analyzed over at least 2 weeks until seven replicates were obtained for each matrix and concentration. Mean recoveries for nearly all VOCs were within 70-130 percent, with $\leq 30$ percent relative standard deviation; these criteria are used to define acceptable performance.

Method detection limits for the heat purgeable and ambient purgeable VOCs were determined using the U.S. Environmental Protection Agency's procedure. Method detection limits ranged from 0.002 to 3 microgram per liter $(\mu \mathrm{g} / \mathrm{L})$ for Method O-4437, heat purgeable VOCs, with reporting levels (RLs) of 0.004-6 $\mu \mathrm{g} / \mathrm{L}$. Method detection limits for Method O-4436, ambient purgeable VOCs, range from 0.007 to $0.1 \mu \mathrm{g} / \mathrm{L}$, with RLs of $0.014-0.2 \mu \mathrm{g} / \mathrm{L}$.

Four ambient purgeable VOCs (1,2-dichloropropane, 1,2-dibromoethane [EDB]), 1,2-dibromo-3-chloropropane [DBCP]), and 1,2,3-trichloropropane) were also included in the heat purgeable method to obtain lower method detection limits. The RLs for these four fumigant compounds decreased by more than an order of magnitude. The RLs for the fumigant compounds range from 0.026 to $0.4 \mu \mathrm{g} / \mathrm{L}$ using the Method O-4127-96 (NWQL Laboratory Schedule [LS] 2020). Reporting levels are $0.018 \mu \mathrm{g} / \mathrm{L}$ for EDB and $0.03 \mu \mathrm{g} / \mathrm{L}$ for DBCP for U.S. Environmental Protection Agency Method 504.1 (NWQL LS 1306). The RLs range from 0.004 to $0.020 \mu \mathrm{g} / \mathrm{L}$, using Method O-4437 (NWQL LS 4437). Method O-4437 replaces NWQL LS 1306 for the fumigant compounds, eliminating a solvent extraction method and providing lower RLs. 
Compounds are reported if the mass spectral identification criteria are met, including concentrations below the RL, except for blank-limited compounds. Blank-limited compounds are not reported below the minimum reporting level concentration. 2-Ethyl-1-hexanol is reported as a blank-limited compound on Method O-4437. Chloromethane, methylene chloride, and toluene have historically been reported as blank-limited compounds in Method O-4127 and will be continued as such in Method O-4436.

Holding time studies indicate overall compound stability in reagent water when samples are preserved to $\mathrm{pH} 2$ with $\mathrm{HCl}(1: 1)$ and water, chilled to 4 degrees Celsius \pm 2 degrees Celsius, and analyzed within 14 days of sampling. Studies indicate that many of the compounds are stable for longer than 14 days. Methyl oxirane, oxirane, and 2-chloromethyl oxirane were incompatible with the acid preservation and subsequently were eliminated from the heat purgeable method. Acrolein, with a calculated ASTM holding time of 7 days, was eliminated from the heat purgeable method due to instability and inconsistent performance. Benzyl chloride was eliminated from the ambient purgeable method because of its short half-life in water samples. Hexane, with a holding time of 6 days, was retained in the method, reported with an estimated remark code of "E" until further data are collected and evaluated.

Four semivolatile compounds (2-chloronaphthalene, 2,6-di-tert butyl phenol, 1,2-dimethylnaphthalene, and 1,6-dimethylnaphthalene) initially demonstrated acceptable performance. Analysis of samples over 18 months on multiple instruments has shown higher variability and unacceptable levels of carryover contamination after the high-concentration calibration standards. The initial method detection limits of $0.08-0.1 \mu \mathrm{g} / \mathrm{L}$ were not representative of day-to-day performance. Consequently, the semivolatile compounds were eliminated from Method O-4436, but these compounds should perform much better using a semivolatile method.

Additional environmental samples were submitted, spiked at the laboratory, and held for a minimum of 3 days to simulate transportation and holding time. The mean recoveries for the 0-4437 (NWQL LS 4437) heat purgeable VOCs ranged from 76 to 116 percent, with relative standard deviations $\leq 17$ percent for 23 groundwater and seven surface-water laboratory matrix spikes. The mean recoveries for the ambient purgeable VOCs ranged from 74 to 111 percent with relative standard deviations $\leq 28$ percent for 24 groundwater and four surface-water laboratory matrix spikes.

A few compounds are reported with a qualifier code. Hexachlorocyclopentadiene will be reported as an estimated concentration on Method O-4437 (NWQL LS 4437) because of higher variability in recovery seen in the initial method development. Bromomethane, carbon disulfide, and chloromethane will also be reported as estimated concentrations on Method O-4436 (NWQL LS 4436). Both bromomethane and chloromethane are gases at room temperature and show higher variability in recovery because of their high Henry's Law Constant. Bromomethane, carbon disulfide, and chloromethane also exhibit higher variability when sulfur is present in samples. Hexane was retained in Method 0-4436 (NWQL LS 4436) ambient purgeable method, although reported as estimated concentrations because of the lower recoveries observed in the holding time study, laboratory matrix spikes, and continuing calibration verification standards.

VOCs are ubiquitous in the environment. Minimizing sample exposure to contaminants is essential during sampling, transportation, and analysis. Field blanks and source solution blanks during sampling are critical to assessing whether a sample has been compromised by contamination. Field-submitted replicates and laboratory matrix spikes also provide valuable quality assurance information.

\section{References}

For web addresses that break across two lines of text, remove the unnecessary extra space when typing or copying and pasting the URL to access the web page. Electronic versions of many of the unpublished NWQL documents cited in this publication are available upon request toLabHelp@usgs.gov or 1-866-ASK-NWQL (1-866-275-6975).

ASTM International, 2001, Standard practice for estimation of holding time for water samples containing organic and inorganic constituents, in Annual book of ASTM standards, Section 11, Water and environmental technology: West Conshohocken, Penn., v. 11.01, D4841-88, 6 p.

ASTM International, 2010, Standard practice for performing detection and quantitation estimation and data assessment utilizing DQCALC software, based on ASTM practices D6091 and D6512 of Committee D19 on water: ASTM D7510-10, 2 p., accessed May 27, 2016 at http://www.astm.org/Standards/ D7510.htm.

Barletta, B., Nissenson, P., Meinardi, S., Dabdub, D., Rowland, F.S., VanCuren, R.A., Pederson, J., Diskin, G.S., and Blake, D.R., 2011, HFC-152a and HFC-134a emission estimates and characterization of CFCs, CFC replacements, and other halogenated solvents measured during the 2008 ARCTAS campaign (CARB phase) over the South Coast Air Basin of California: Atmospheric Chemistry and Physics, v. 11, p. 2655-2669.

California Environmental Protection Agency, Office of Environmental Health Hazard Assessment, 2009, Announcement of publication of the final public health goal for 1,2,3trichloropropane in drinking water, accessed November 2013, at http://oehha.ca.gov/water/phg/123tcp082009.html.

Centers for Disease Control and Prevention, Agency for Toxic Substances \& Disease Registry, July 1999, Toxicological profile for $n$-Hexane, chapter 4, accessed on February 4, 2015 at http://www.atsdr.cdc.gov.

Centers for Disease Control and Prevention, National Institute of Occupational Health and Safety, 2015, accessed November 24, 2015 at http://www.cdc.gov/niosh/topics/ chemical-safety/\#msds. 
Childress, C.J., Foreman, W.T., Connor, B.F., and Maloney, T.J., 1999, New reporting procedures based on longterm method detection levels and some considerations for interpretations of water-quality data provided by the U.S. Geological Survey National Water Quality Laboratory: U.S. Geological Survey Open-File Report 99-193, 19 p.

Connor, B.F., Rose, D.L., Noriega, M.C., Murtagh, L.K., and Abney, S.R., 1998, Methods of analysis by the U.S. Geological Survey National Water Quality Laboratory: Determination of 86 volatile organic compounds in water by gas chromatography/mass spectrometry, including detections less than reporting limits: U.S. Geological Survey OpenFile Report 97-829, 78 p., accessed April 11, 2013, at https://pubs.er.usgs.gov/publication/OFR97829.

Environment Canada, Health Canada, 2009, Screening assessment for the challenge, benzene, (chloromethyl)-, (benzyl chloride): Chemical Abstracts Service Registry Number 100-44-7, 31 p., accessed November 18, 2015, at http://www.ec.gc.ca/ese-ees/ default.asp?lang=En\&n=EF864A36-1.

Helsel, D.R., and Hirsch, R.M., 2002, Statistical methods in water resources: U.S. Geological Survey Techniques of Water-Resources Investigations, book 4, chapter A3, 522 p.

Love, J.T., Delzer, G.C., Abney, S.R., Zogorski, J.S., 1998, Study design and analytical results used to evaluate stability of volatile organic compounds in water matrices: U.S. Geological Survey Open-File Report 98-637, 155 p., accessed May 13, 2013, at http://pubs.er.usgs.gov/publication/ ofr98637.

Lee, Jong-Song, 2015, Pentane, all isomers, Development Support Document TCEQ2015 (September 14, 2015), Texas Commission on Environmental Quality, 33 p., accessed February 4, 2016, at http://www.tceq.texas.gov/assets/ public/implementation/tox/dsd/final/pentane.pdf.

Midgley, P.M., Fisher, D.A., 1993, The production and release to the atmosphere of chlorodifluoromethane (HCFC 22): Atmospheric Environment, v. 27A, no. 14, p. 2215-2223.

Munch, J.W., 1995, Method 524.2-Measurement of purgeable organic compounds in water by capillary gas chromatography/mass spectrometry, revision 4.1: Cincinnati, Ohio, Environmental Monitoring Systems Laboratory, U.S. Environmental Protection Agency, 48 p.

Munch, J.W., and Grimmett, Paul E., 2008, Method 522 determination of 1,4-dioxane in drinking water by solid phase extraction (SPE) and gas chromatography/mass spectrometry (GC/MS) with selected ion monitoring (SIM), version 1.0: Cincinnati, Ohio, National Exposure Research Laboratory, Office of Research and Development, U.S. Environmental Protection Agency, 41 p.

National Environmental Laboratory Accreditation Conference, 2003, Appendix A-Glossary, chap. 1, p. 1A-1-1A-14, in NELAC standard, 2003, EPA/600/R-04/003, accessed May 27, 2016, at http://nelac-institute.org/ docs/2003nelacstandard.pdf.
National Institute of Standards and Technology, 2000, NIST/ SEMATECH e-Handbook of statistical methods, accessed February 4, 2016, at http://www.itl.nist.gov/div898/ handbook/.

New Jersey Department of Environmental Protection, 2008, Ground water quality standard for 2-ethyl-1-hexanol CASRN 104-76-7, 5 p., accessed April 30, 2014, at http://nj.gov/dep/wms/bears/docs/2-ethyl-1-hexanol.pdf.

Olsen, L.D., Valder, J.F., Carter, J.M., and Zogorski, J.S., 2013, Prioritization of constituents for national- and regionalscale ambient monitoring of water and sediment in the United States: U.S. Geological Survey Scientific Investigations Report 2012-5218, 203 p., accessed May 7, 2014, at http://pubs.usgs.gov/sir/2012/5218/.

Prakash, B., Zaffiro, A.D., Zimmerman, M., Munch, D.J., and Pepich, B.V., 2009, Method 524.3-Measurement of purgeable organic compounds in water by capillary column gas chromatography/mass spectrometry, U.S. Environmental Protection Agency publication number EPA 815-B-09009: Cincinnati, Ohio, Office of Groundwater and Drinking Water, Technical Support Center, U.S. Environmental Protection Agency, v. 1.0, June 2009, p. 524.3-1-524.3-55, accessed November 18, 2015, at https://www.nemi.gov/ methods/method_summary/10417/.

PubChem, 2014, Compound summary for CID 6368, 1,1-difluroethane: PubChem accessed May 14, 2014, at http://pubchem.ncbi.nlm.nih.gov/rest/chemical/difluoroethane.

Rose, D.L., and Sandstrom, M.W., 2003, Methods of analysis by the U.S. Geological Survey National Water Quality Laboratory-Determination of gasoline oxygenates, selected degradates and BTEX in water by heated purge-and-trap/ gas chromatography/mass spectrometry: U.S. Geological Survey Water-Resources Investigations Report 03-4079, 31 p., accessed April 11, 2013, at https://pubs.er.usgs.gov/ publication/wri034079.

SRC, 2013, Physical properties database: SRC, accessed March 20, 2013, at http://www.srcinc.com.

Taylor, J.K., 1987, Quality assurance of chemical measurements: Chelsea, Mich., Lewis Publishers, 328 p.

Taylor, J.K., 1990, Statistical techniques for data analysis: Boca Raton, Fla., CRC Press, 200 p.

Thiros, S.A., Bender, D.A., Mueller, D.K., Rose, D.L., Olsen, L.D., Martin, J.D., Bernard, Bruce, Zogorski, J.S., 2011, Design and evaluation of a field study on the contamination of selected volatile organic compounds and wastewater-indicator compounds in blanks and groundwater samples. U.S. Geological Survey Scientific Investigations Report 2011-5027, 85 p., accessed May 7, 2014, at http://pubs.usgs.gov/sir/2011/5027/. 
Tou, J.C., Kallos, G.J., 1974, Kinetic study of the stabilities of chloromethyl methyl ether and bis (chloromethyl) ether in humid air: Analytical Chemistry, v. 46, no. 12, p. 1866-1869.

U.S. Environmental Protection Agency, 1984, Method 603: Acrolein and acrylonitrile, Appendix A to Part 136, Methods for organic chemical analysis of municipal and industrial wastewater, 21 p., accessed February 24, 2016, at http://www.epa.gov/sites/production/files/2015-08/ documents/method_603_1984.pdf.

U.S. Environmental Protection Agency, 1995, Method 504.1, 1,2-dibromoethane (EDB), 1,2-dibromo-3-chloropropane (DBCP), and 1,2,3-trichlororpropane (123TCP) in water by microextraction and gas chromatography revision 1.1, National Exposure Research Laboratory, Office of Research and Development, Munch, J.W., ed., 20 p., accessed November 18, 2015, at https://www.nemi.gov/methods/method_summary/4825/.

U.S. Environmental Protection Agency, 2002, Guidelines establishing test procedures for the analysis of pollutants (Part 136, Appendix B. Definition and procedure for the determination of the method detection limit-Revision 1.11): U.S. Code of Federal Regulations, Title 40, revised as of July 1, 2002, p. 635-638.

U.S. Environmental Protection Agency, 2012, The third unregulated contaminant monitoring rule (UCMR 3), Office of Water: EPA 815-F-12-002, May 2012, accessed November 19, 2013, at http://water.epa.gov/lawsregs/ rulesregs/sdwa/ucmr/ucmr3/upload/UCMR3_FactSheet_ General.pdf.
U.S. Environmental Protection Agency, 2014, Technical fact sheet-1,4-Dioxane, EPA 505-F-14-011, Office of Solid Waste and Emergency Response (5106P), accessed on January 13, 2016 at URL http:/www.epa.gov/sites/ production/files/2014-03/documents/ffrro_factsheet contaminant_14-dioxane_january2014_final.pdf.

U.S. Geological Survey, 2006, Collection of water samples (ver. 2.0): U.S. Geological Survey Techniques of WaterResources Investigations, book 9, chap. A4, accessed March 31, 2015, at http://pubs.water.usgs.gov/twri9A4/.

U.S. National Library of Medicine, 2015, U.S. National Library of Medicine TOXNET toxicological data network, accessed November 24, 2015, at http://toxnet.nlm.nih.gov.

Wilde, F.D., Radtke, D.B., Gibs, Jacob, and Iwatsubo, R.T., 1999, Preparations for water sampling, in National field manual for the collection of water-quality data: U.S. Geological Survey Techniques of Water-Resources Investigations, book 9, chap. A1, accessed May 13, 2013, at http://pubs.water.usgs.gov/twri9A1.

Wilde, F.D., ed., 2004, Cleaning of equipment for water sampling (ver. 2.0): U.S. Geological Survey Techniques of Water-Resources Investigations, book 9, chap. A3, accessed March 31, 2015, at http://pubs.water.usgs.gov/twri9A3/.

Wilde, F.D., Radtke, D.B., Gibs, Jacob, and Iwatsubo, R.T., eds., 2004 with updates through 2009, Processing of water samples (ver. 2.2): U.S. Geological Survey, Techniques of Water-Resources Investigations, book 9, chap. A5, April 2004. A5, accessed March 31, 2015, at http://pubs.water.usgs.gov/ twri9A5/. 


\section{Determination of Heat Purgeable and Ambient Purgeable Volatile Organic Compounds in Water}

\section{Tables}

[Click on table title to access Excel file]

Table 3. Suggested concentrations of heat purgeable volatile organic compounds in the primary and secondary calibration standards, the calibration range in water, and the concentration of surrogate and internal standard compounds for Method 0-4437, grouped by primary calibration standard mix.

Table 4. Suggested concentration of ambient purgeable volatile organic compounds in the primary calibration standards, the calibration range in water, and the concentration of the surrogate and internal standard compounds for Method 0-4436.

Table 5. Summary of suggested purge-and-trap sample concentrator, gas chromatograph, and mass spectrometer operating conditions for heat purgeable volatile organic compounds, Method 0-4437.

Table 6. Internal standard compound assignment, quantitation ion, and primary and secondary qualifying ions for heat purgeable volatile organic compounds in Method 0-4437, listed in chromatographic retention time order.

Table 7. Summary of suggested purge-and-trap sample concentrator, gas chromatograph, and mass spectrometer operating conditions for ambient purgeable volatile organic compounds for Method 0-4436.

Table 8. Internal standard compound assignment, quantitation ion, and primary and secondary qualifying ions for ambient purgeable volatile organic compounds for Method 0-4436, listed in chromatographic retention time order.

Table 9. Tuning performance criteria for mass spectrometer using 1-bromo-4-fluorobenzene.

Table 10. Suggested instrument batch run sequence.

Table 11. Method detection limits and reporting levels for heat purgeable volatile organic compounds, Method 0-4437.

Table 12. Method detection limits and reporting levels for ambient purgeable volatile organic compounds, Method 0-4436.

Table 13. Percent recovery and variability for heat purgeable volatile organic compounds in volatile blank water, groundwater, and surface water for seven replicates, spiked at two concentrations, and preserved to pH 2 with hydrochloric acid (1:1), Method 0-4437.

Table 14. Percent recovery and variability for ambient purgeable volatile organic compounds in volatile blank water, groundwater, and surface water for seven replicates, spiked at two concentrations, and preserved to pH 2 with hydrochloric acid (1:1), Method 0-4436.

Table 15. Percent recovery and variability for a 28-day holding time study for heat purgeable volatile organic compounds in volatile blank water, adjusted to $\mathrm{pH} 2$ with hydrochloric acid (1:1), and stored at 4 plus or minus 2 degrees Celsius, Method 0-4437.

Table 16. Summary of parameters used to calculate holding times from a 28-day study for heat purgeable volatile organic compounds in volatile blank water, preserved to $\mathrm{pH} 2$ with hydrochloric acid (1:1), and stored at 4 plus or minus 2 degrees Celsius, using American Standard for Testing and Materials Procedure D4841-88, Method 0-4437.

Table 17. Percent recovery and variability for a 28-day holding time study for ambient purgeable volatile organic compounds in volatile blank water, adjusted to $\mathrm{pH} 2$ with hydrochloric acid (1:1), and stored at 4 plus or minus 2 degrees Celsius, Method 0-4436.

Table 18. Summary of parameters used to calculate holding times from a 28-day study for ambient purgeable volatile organic compounds in volatile blank water, preserved to $\mathrm{pH} 2$ with hydrochloric acid (1:1), and stored at 4 plus or minus 2 degrees Celsius, using American Standard for Testing and Materials Procedure D4841-88, Method 0-4436.

Table 19. Percent recovery and variability of heat purgeable volatile organic compounds from 75 continuing calibration verification standards, spiked in volatile blank water, preserved to $\mathrm{pH} 2$ with hydrochloric acid (1:1), and determined from January through August 2013, Method 0-4437.

Table 20. Summary of heat purgeable volatile organic compounds detected in laboratory reagent-water blanks determined from January through August 2013, Method 0-4437. 
Table 21. Summary of heat purgeable volatile organic compounds detected in 86 field-submitted blanks determined from January through August 2013, Method 0-4437.

Table 22. Summary of environmental sample analyses in groundwater and surface water for heat purgeable volatile organic compounds from January through August 2013, Method 0-4437.

Table 23. Percent recovery and variability of heat purgeable volatile organic compounds for 30 laboratory groundwater and surface water matrix spikes, Method 0-4437.

Table 24. Summary of National Water Quality Laboratory schedules and analytical methods for volatile organic compounds.

Table 25. Summary of paired environmental sample determinations for four fumigant compounds using the heated purge method compared to the ambient purge method.

Table 26. Percent recovery and variability of ambient purgeable volatile organic compounds for continuing calibration verification standards, spiked at 0.5 micrograms per liter, and determined by Method 0-4436.

Table 27. Summary of ambient purgeable volatile organic compound detections in laboratory reagent-water blanks determined from January through August 2013, Method 0-4436.

Table 28. Summary of ambient purgeable volatile organic compound detections in field-submitted blanks determined from January through August 2013, Method 0-4436.

Table 29. Summary of environmental sample analyses in groundwater and surface water for ambient purgeable volatile organic compounds determined from January through August 2013, Method 0-4436.

Table 30. Percent recovery and variability of ambient purgeable volatile organic compounds for 28 laboratory groundwater and surface water matrix spikes, Method 0-4436.

Table 31. Wilcoxon rank sum test of whether the median recoveries in ambient purgeable volatile organic compounds in continuing calibration verification standards determined by Method 0-4436 are the same as those determined by Method 0-4127.

Table 32. Levene test and F-test of whether the variability of recoveries of ambient purgeable volatile organic compounds in continuing calibration verification standards determined by Method 0-4436 are the same as those determined by Method 0-4127. 



\section{Glossary}

\section{B}

Bias Systematic error inherent in a method or measurement system. The error can be positive (for example, contamination or spectral interference) or negative (for example, analyte loss or signal suppression) (Taylor, 1987). It differs from random error, which shows no such consistent or systematic deviation. Bias is the preferred term used by the NWQL.

Blank-limited compound A compound that is frequently detected in laboratory reagentwater blanks.

\section{C}

Carryover blank (COB) A COB is prepared from VBW and placed in an instrument batch run after higher-concentration quality-control samples (calibration standards, CCVs, SPKs, MSPKs), and (or) highly contaminated samples. The purpose of the $\mathrm{COB}$ is reduce residual VOCs in the purge and trap system and prevent cross contamination of the next determination. $\mathrm{COBs}$ are not preserved. $\mathrm{COB}$ data are not reported to the database.

\section{1}

Internal standard compound A known amount of a standard added to a sample and carried through the entire measurement process as a reference for evaluating and controlling the bias and variability of the applied analytical method (in part from National Environmental Laboratory Accreditation Conference, 2003). Isotope-labeled VOCs or other similar VOCs, that are unlikely to be found in environmental samples, are selected for internal standard compounds.

\section{L}

Laboratory reagent-water blank (LRB) A reagent water sample processed through the sample preparation and analysis steps of the analytical process used to measure bias of the analytical method. An LRB is synonymous with an instrument reagent blank for the VOC methods.

Laboratory reagent-water spike (SPK) standard A synthetic matrix fortified with known concentrations of all, or a representative selection of the method compounds. The synthetic matrix usually is the same as the same as the method blank, for example, VBW, for VOC determinations. The SPK is prepared at the laboratory prior to analysis. EPA uses the term "laboratory fortified blank" for this type of sample.

\section{M}

Matrix spike (MSPK) An environmental sample fortified with known concentrations of all, or a representative selection of the method compounds, used to measure bias and variability because of sample matrix interferences. The sample may be fortified at the collection site (field matrix spike) or at the laboratory (laboratory matrix spike). Laboratory matrix spikes are fortified upon receipt at the laboratory and analyzed within 14 days of sample collection.

Method detection limit (MDL) The MDL is the minimum concentration of a compound that can be identified, measured, and reported with 99 percent confidence that the compound concentration is greater than zero. At the MDL the risk of a false positive (reporting a detection when no compound is present) is predicted to be less than 1 percent. The MDLs in this report were determined using the EPA procedure (U.S. Environmental Protection Agency, 2002), and Childress, and others, 1999. The ASTM procedure (ASTM International, 2010) utilizing DQCALC software is being investigated as an additional method for determining MDLs.

Minimum reporting level (MRL) The MRL is the smallest measured concentration of an analyte that may be reliably reported. Because the definition of the MRL is not specific, an MRL can be set at a concentration acceptable to the data user and the laboratory as long as a reliable measurement is achieved (Childress and others, 1999).

\section{R}

Reagent water Deionized water prepared by distillation, ion exchange, and filtration. The deionized water was then pumped through charcoal prefilters and a dual wavelength ultraviolet oxidizer lamp for removal of trace organic compounds to produce high-purity, organic-free reagent water. 
Reporting level (RL) The RL is set at twice the MDL. The RL is used to control false negative error-the risk of a false negative (not detecting a compound when present) is less than 1 percent at the RL (Childress and others, 1999).

\section{S}

Surrogate standard compound A substance with properties that mimic the compound (s) of interest. It is unlikely to be found in environmental samples and is added to them for quality-control purposes (National Environmental Laboratory Accreditation Conference, 2003). Isotope-labeled VOCs or other similar VOCs are selected for surrogate standard compounds.

\section{T}

Third party check (TPC) standard An analytical standard prepared by an independent source - different from that used to prepare the calibration and spike standards. The third party check standard is used to verify that the calibration standards are accurate and have not changed as a result of degradation or errors in preparation.

\section{V}

Variability Random error in independent measurements as the result of repeated application of the process under specific conditions. Variability can be statistically described by the standard deviation (standard error) (Taylor, 1990). All data contain some experimental error and individual measurements change or fluctuate within limits. Precision is a measure of variability in experimental data. Variability is the preferred term used by the NWQL.

Volatile blank water (VBW) Reagent water that has been boiled for $1 \mathrm{hr}$ and purged continuously with ultra-high purity nitrogen gas to remove volatile organic contaminants. VBW is used to prepare the COBs, LRBs, and standard solutions. 


\section{Appendix 1}

[Click on table title to access Excel file]

Table 1-1. Volatile organic compounds discontinued from the ambient purgeable method for routine determination for the National Water-Quality Assessment Program.

Table 1-2. Physical properties of compounds ranked as priority compounds by the National Water-Quality Assessment Program for purgeable analytical methods. 
Publishing support provided by:

Denver Publishing Service Center, Denver, Colorado

For more information concerning this publication, contact:

Chief, USGS National Water Quality Laboratory

Box 25585, Mail Stop 407

Denver, C0 80225-0585

(303) 236-2000

Or visit the National Water Quality Laboratory Web site at:

http://nwql.usgs.gov/

This publication is available online at:

http://dx.doi.org/10.3133/tm5B12 



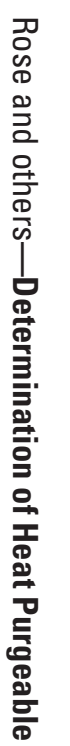

登

言

동.

壳

通

ऽ

을

옹.

응

틈

क.

$\sum_{0}$

蛋

$\rightarrow$

$\frac{1}{3}$

品

象

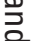

3

票

क

罢 